\title{
Deformations of special geometry: in search of the topological string
}

\author{
G.L. Cardoso, ${ }^{a}$ B. de $\mathbf{W i t}^{b, c}$ and S. Mahapatra ${ }^{d}$ \\ ${ }^{a}$ Center for Mathematical Analysis, Geometry and Dynamical Systems, \\ Department of Mathematics, Instituto Superior Técnico, Universidade de Lisboa, \\ Av. Rovisco Pais, 1049-001 Lisboa, Portugal \\ ${ }^{b}$ Nikhef, Science Park 105, 1098 XG Amsterdam, The Netherlands \\ ${ }^{c}$ Institute for Theoretical Physics, Utrecht University, \\ Leuvenlaan 4, 3584 CE Utrecht, The Netherlands \\ ${ }^{d}$ Physics Department, Utkal University, \\ Bhubaneswar 751004 , India \\ E-mail: gcardoso@math.ist.utl.pt, B.deWit@uu.nl, swapna@iopb.res.in
}

ABSTRACT: The topological string captures certain superstring amplitudes which are also encoded in the underlying string effective action. However, unlike the topological string free energy, the effective action that comprises higher-order derivative couplings is not defined in terms of duality covariant variables. This puzzle is resolved in the context of real special geometry by introducing the so-called Hesse potential, which is defined in terms of duality covariant variables and is related by a Legendre transformation to the function that encodes the effective action. It is demonstrated that the Hesse potential contains a unique subsector that possesses all the characteristic properties of a topological string free energy. Genus $g \leq 3$ contributions are constructed explicitly for a general class of effective actions associated with a special-Kähler target space and are shown to satisfy the holomorphic anomaly equation of perturbative type-II topological string theory. This identification of a topological string free energy from an effective action is primarily based on conceptual arguments and does not involve any of its more specific properties. It is fully consistent with known results. A general theorem is presented that captures some characteristic features of the equivalence, which demonstrates at the same time that nonholomorphic deformations of special geometry can be dealt with consistently.

KEYWords: Supersymmetry and Duality, Extended Supersymmetry, Topological Strings, Supersymmetric Effective Theories

ARXIV EPRINT: 1406.5478 


\section{Contents}

1 Introduction 1

$\begin{array}{llr}2 & \text { Real and deformed special geometry } & 8\end{array}$

3 The generic structure of the Hesse potential 12

4 Non-holomorphic deformations and the anomaly equation 20

$\begin{array}{lll}5 & \text { Evaluating the third-order contributions } & 27\end{array}$

6 Summary and conclusions 31

A Non-holomorphic deformation of special geometry 33

$\begin{array}{lll}\text { A.1 Theorem } & 33\end{array}$

A.2 Proof 34

A.3 Corollary 35

B The symplectic functions $\mathcal{H}_{i}^{(a)}$ for $a \geq 2$ and some other functions that $\begin{array}{ll}\text { do not initially appear in } \mathcal{H} & 36\end{array}$

$\begin{array}{lll}\text { C Transformation rules of } \omega_{I} \text { and } \omega_{I J} \text { to order } \alpha^{2} & 38\end{array}$

D Topological free energies for genus $g \leq 3$ that satisfy the holomorphic $\begin{array}{lr}\text { anomaly equation } & 39\end{array}$

E An application: the FHSV model 41

\section{Introduction}

As is well known, Lagrangians for $N=2$ supersymmetric vector multiplets are encoded in a holomorphic function $F(X)$ whose arguments correspond to the complex scalar fields $X^{I}$ of the vector multiplets. These Lagrangians often play a role as Wilsonian effective field theories that describe the physics below a certain mass scale. Homogeneity of the holomorphic function is required whenever the vector multiplets are coupled to supergravity [1]. The physical vector multiplet scalars are then projectively defined in terms of these variables as a result of the local scale and $U(1)$ invariance of the description used in [1]. It is possible that the function depends, in addition, on one or more holomorphic fields, possibly associated with some other chiral multiplets. An example of this is the so-called Weyl multiplet that describes the pure supergravity degrees of freedom [2]. When the function $F$ depends on the Weyl multiplet, then it will also encode a class of higher-derivative 
couplings. ${ }^{1}$ In case these higher-order derivative couplings are absent, we will denote the function by $F^{(0)}$, which is always holomorphic and homogeneous and encodes an action that is at most quadratic in space-time derivatives. This action will henceforth be referred to as the 'classical action', and its associated non-linear sigma model parametrizes a special-Kähler space.

The abelian vector fields in these actions are subject to electric/magnetic duality under which the electric field strengths and their duals transform under symplectic rotations. It is then possible to convert to a different duality frame, by regarding half of the rotated field strengths as the new electric field strengths and the remaining ones as their duals. The latter are then derivable from a new action. To ensure that the characterization of the new action in terms of a holomorphic function remains preserved, the scalars of the vector multiplets are transformed correspondingly. This amounts to rotating the complex fields $X^{I}$ and the holomorphic derivatives of the underlying function $F(X)$ by the same symplectic rotation as the field strengths and their dual partners $[1,5]$. For reasons that will be described shortly, we shall refer to the array $\left(X^{I}, F_{J}\right)$ as the period vector, where $F_{J}(X)=\partial_{J} F(X)$. The indices $I, J$ label the vector multiplets, and cover the range $I, J=$ $0,1, \ldots, n$, so that the period vector has (complex) dimension $2(n+1)$. Electric/magnetic duality thus constitutes a group of equivalence transformations that relate two different Lagrangians (based on two different functions) giving rise to an equivalent set of equations of motion and Bianchi identities. A subgroup of these equivalence transformations may constitute an invariance group, meaning that the Lagrangian and its underlying function $F(X)$ remain unchanged. We stress that the latter two quantities do not transform as a function under these equivalence transformations.

As it turns out one encounters a similar situation when studying Calabi-Yau threefolds. The moduli space of these three-folds is a local product of two submanifolds, describing the metric deformations of the complex structure and of the Kähler class, respectively. The complex structure moduli determine the shape, and the Kähler moduli the size of the Calabi-Yau three-folds. Usually, when referring to the Calabi-Yau moduli space, one refers to either one of these two submanifolds. As it turns out, the corresponding metric deformations are related to the odd and even harmonic forms, respectively, and the number of moduli is thus determined by the topology of the Calabi-Yau three-folds (see e.g. [6]). The latter is specified by the Hodge numbers $h^{p, \bar{p}}$ which specify the number of independent $(p, \bar{p})$ harmonic forms. The odd harmonic forms consist of a $(3,0)$ holomorphic form $\Omega$, and $h^{2,1}(2,1)$-forms, as well as their conjugate $(0,3)$ - and $(1,2)$-forms. Under an infinitesimal change of the complex structure the (3,0)-form $\Omega$ changes into $\Omega$ and the $(2,1)$-forms, which leads to its periods, in the following way,

$$
X^{I}=\oint_{A^{I}} \Omega, \quad F_{I}^{(0)}=\oint_{B_{I}} \Omega .
$$

Here $A^{I}$ and $B_{I}$ are an integral basis of homology 3-cycles (in a symplectic basis) dual to the three-forms. The index $I$ takes the values $I=0,1, \ldots, h^{2,1}$, corresponding to the $(3,0)$ -

\footnotetext{
${ }^{1}$ These actions are all based on a chiral superspace density, but other $N=2$ supersymmetric higherderivative couplings are known to exist (see e.g. [3, 4]). The latter will not be considered in this paper.
} 
and the $(2,1)$-forms and their conjugates. The $X^{I}$ (or alternatively the $F^{(0)} I$ ) projectively parametrize the complex-structure deformations, so that the complex dimension of the corresponding moduli space equals $h^{2,1} .^{2}$

For the periods one can also show (at least in a suitable homology basis) that there exists a holomorphic, homogeneous, function $F^{(0)}(X)$, such that $F^{(0)}{ }_{I}=\partial_{I} F^{(0)}(X) .{ }^{3}$ The duality transformations on the periods simply arise from symplectic redefinitions of the homology basis of the 3-cycles. It is worth pointing out that in this case one is dealing with discrete symplectic transformations, while in the supergravity case the transformations are continuous (unless one has to account for an integral lattice of electric and magnetic charges). This particular geometry with its associated period vectors and symplectic transformations is known as special geometry [7] (for a review, see [8]).

The Calabi-Yau moduli space and the supergravity action describing a Calabi-Yau string compactification are related, because the target space metric associated with the non-linear sigma model contained in the corresponding Wilsonian effective action of vector multiplets coupled to supergravity, must be equal to the metric of the Calabi-Yau moduli space [9]. This target space is a so-called special-Kähler space, whose Kähler potential is proportional to [1],

$$
K(t, \bar{t}) \propto \log \left[\frac{\mathrm{i}\left(X^{I} \bar{F}^{(0)} I-\bar{X}^{I} F^{(0)} I\right)}{\left|X^{0}\right|^{2}}\right],
$$

and $F^{(0)}(X)$ is the holomorphic function that determines the supergravity action quadratic in space-time derivatives. Because $F^{(0)}(X)$ is homogeneous of second degree, this Kähler potential depends only on the 'special' coordinates $t^{i}=X^{i} / X^{0}$ and their complex conjugates, where $i=1, \ldots, n$, so that we are dealing with a special-Kähler space of complex dimension $n$. In view of the homogeneity, the symplectic rotations acting on the period vector $\left(X^{I}, F^{(0)}{ }_{I}\right)$ induce corresponding (non-linear) transformations on the special coordinates $t^{i}$. Up to a Kähler transformations, the Kähler potential transforms as a function under duality.

Yet another quantity that reflects the geometrical features of the Calabi-Yau moduli space is the topological string. Perturbative string theory is defined in terms of maps from Riemann surfaces $\Sigma_{g}$ to a target space. When the worldsheet theory has $(2,2)$ supersymmetry and the target space is Ricci flat, one may construct a topological version of perturbative string theory by a procedure called twisting [10]. The resulting theory is a cohomological theory, and correlation functions of observables are independent of the wordsheet metric on $\Sigma_{g}$. When the target-space is a Calabi-Yau three-fold, the twisted theory is called topological string theory [11]. There exist two versions of topological string theory, called the A- and the B-models. In the A-model, the correlation functions depend only

\footnotetext{
${ }^{2}$ For completeness we mention that there exists an analogous construction for the Kähler moduli. While the complex structure moduli are associated with the odd cohomology class, $\mathbb{H}^{(3,0)} \oplus \mathbb{H}^{(2,1)} \oplus \mathbb{H}^{(1,2)} \oplus \mathbb{H}^{(0,3)}$, the Kähler moduli are associated with the even class, $\mathbb{H}^{(0,0)} \oplus \mathbb{H}^{(1,1)} \oplus \mathbb{H}^{(2,2)} \oplus \mathbb{H}^{(3,3)}$. The corresponding $2+2 h^{1,1}$ coordinates projectively describe the $h^{1,1}$ complex Kähler moduli [6].

${ }^{3}$ The functions $F^{(0)}$ encoding the moduli space geometry of Calabi-Yau three-folds correspond to a restricted class. This paper pertains to functions belonging to a more general class.
} 
on the Kähler moduli, while in the B-model they only depend on the complex structure moduli.

The topological string is defined in terms of topological free energies $F^{(g)}$, which are computed from suitable correlators on orientable Riemann surfaces $\Sigma_{g}$ of genus $g$. These free energies may be formally combined into one single object $F(X, \lambda)$, the free energy of topological string theory, which has the asymptotic expansion,

$$
F(X, \lambda)=\sum_{g=0}^{\infty} \lambda^{g-1} F^{(g)}(X),
$$

with $\lambda$ playing the role of a formal (complex) expansion parameter. This expression, being a perturbative series in $\lambda$, is expected to receive non-perturbative corrections in $\lambda[12-$ 14]. Note that we have (tentatively) included $F^{(0)}(X)$ in (1.3), which is the function that encodes the Calabi-Yau moduli space metric. The functions $F^{(g)}(X)$ are homogeneous functions of the $X^{I}$ of degree $-2(g-1)$, so that the $X^{0}$-dependence can be scaled out and subsequently be absorbed into the expansion parameter $\lambda$. In this way $F\left(t, \lambda^{\prime}\right)=$ $\left(X^{0}\right)^{-2} F(X, \lambda)$ with $\lambda^{\prime}=\lambda /\left(X^{0}\right)^{2}$, and $F^{(g)}(t)=\left(X^{0}\right)^{2 g-2} F^{(g)}(X)$. This suggests a role of $\lambda^{\prime}$ as a loop-counting parameter with $\left(\lambda^{\prime}\right)^{-1} F^{(0)}(t)$ equal to the classical free energy.

It may seem tempting to identify the expansion (1.3) in this way with the similar expansion of the effective action in terms of $W^{2}$, the square of the lowest component of the Weyl multiplet, thus generating higher-derivative couplings in the action. However, this interpretation is inconsistent with the behaviour one expects for the free energy of the topological string. The reason is that the genus- $g$ free energies should be consistent with dualities induced by symplectic rotations of the periods $\left(X^{I}, F^{(0)} I\right)$ of the underlying Calabi-Yau moduli space. In particular, the $F^{(g)}(X)$ (with $g>0$ ) should transform as functions under these dualities, and the $F^{(g)}(t)$ as sections. Note that this does not apply to $F^{(0)}$, which does not transform as a function under electric/magnetic duality. At this point one concludes that it was premature to include $F^{(0)}$ into the free energy of the topological string, as the genus- $g$ contributions with $g>0$ behave as functions under duality, while $F^{(0)}$ does not.

On the other hand, the Wilsonian action encoded by the similar expansion,

$$
F\left(X, W^{2}\right)=\sum_{g=0}^{\infty}\left(W^{2}\right)^{g} F^{(g)}(X),
$$

is subject to different duality transformations, namely those induced by rotations of the full period vector $\left(X^{I}, F_{I}\right)$ rather than of the 'classical' period vector $\left(X^{I}, F^{(0)}\right)$. Consequently the corresponding coordinates $X^{I}$ will transform differently under duality, so that one must conclude that the $X^{I}$ appearing in the topological string free energy and the $X^{I}$ appearing in the Wilsonian action cannot be identical variables. Hence the coefficient functions $F^{(g)}$ for the Wilsonian action appearing in (1.4) that multiply even powers of the Weyl multiplet are not transforming as functions under duality, unlike those of the topological string. This aspect is most striking when considering duality symmetries such as S- and T-duality. Under these dualities the functions $F^{(g)}$ of the topological string are invariant 
(possibly up to a scale factor), whereas the analogous coefficient functions of the Wilsonian action (1.4) transform non-linearly and are not invariant. Hence, in spite of the similarity of the expansions, there is no ground for assuming that the coefficient functions $F^{(g)}$ of the topological string will coincide with the corresponding coefficient functions appearing in the expression (1.4) that encodes the effective action. This observation was already made in e.g. $[15,16]$, where its consequences were investigated for dualities that define symmetries of the model. Nevertheless, we should stress that there must exist a relation between the effective action and the topological string in view of the fact that the topological string does capture certain contributions to string amplitudes, which must in turn be reflected in the effective action $[11,17]$.

We thus conclude that one seems to be dealing with two different series expansions of the form (1.3) and (1.4), one pertaining to the topological string free energy and another one to the effective Wilsonian action with a class of higher-derivative coupings. In spite of their qualitatively different behaviour with respect to duality they should somehow describe the same physics. To make matters more subtle, it is known that both the topological string and the effective supergravity action are subject to non-holomorphic modifications. Hence it is reasonable to expect that these modifications are therefore related as well. However, so far non-holomorphic deformations have not been incorporated in the standard treatment of special geometry. The non-holomorphic modification in the effective action is due to the integration over massless modes [18], whereas those in the topological string free energy originate from the pinching of cycles of the Riemann surfaces [19]. The need for non-holomorphic corrections can often be deduced from the lack of invariance under integer-valued duality symmetries, which requires modular functions that are not fully holomorphic. This was also observed when calculating the entropy for BPS black holes with S-duality invariance [20].

In this paper we will systematically study the connection between the effective action and the topological string. ${ }^{4}$ Here we should stress that we are just referring to functions that can potentially define the topological string free energy in relation to an underlying effective action. Whether these functions will actually have a topological string realization is a priori not known. But the connection that is proposed in this paper seems to be universal so that it will apply also to those cases where a topological string realization does exist. We will start from the holomorphic function that encodes the Wilsonian action, and construct another quantity that transforms in the same way under duality as the topological string free energy. Here we are inspired by previous work on BPS black holes [15, 16, 22], where the so-called Hesse potential emerged as the relevant quantity, defined in the context of real special geometry [23-25]. The Hesse potential transforms as a (real) function under duality. It is related to the function $F(X)$ that encodes the effective action via a Legendre transform and it is expressed in terms of duality covariant variables, so that its behaviour under duality is comparable to what one observes for the topological string. In hindsight it is not so difficult to understand this relation by reflecting on the more familiar case of four-dimensional abelian gauge fields, where the Lagrangian is a function of the abelian

\footnotetext{
${ }^{4}$ A preliminary account of our results was published in the proceedings of the Frascati School 2011 on Black Objects in Supergravity [21].
} 
field strengths $F_{\mu \nu}$ and possibly other fields (that we assume to be electrically neutral). The expressions for the dual field strengths, which are related to the derivative of the full Lagrangian with respect to the original field strength, do depend on the specific interaction terms contained in the Lagrangian. Therefore the electric/magnetic duality transformation rules for the original field strengths will depend on the details of the underlying Lagrangian. On the other hand the Hamilonian depends on different quantities, namely the spatial part of the gauge potential $\boldsymbol{A}$ and the electric displacement field $\boldsymbol{D}$, where the latter follows from taking the derivative of the Lagrangian with respect to $\boldsymbol{E}$. The precise definition of $\boldsymbol{D}$ will thus implicitly depend on the details of the Lagrangian. Under electric/magnetic duality $\boldsymbol{B} \propto \boldsymbol{\nabla} \times \boldsymbol{A}$ and $\boldsymbol{D}$ transform as a dual pair and the Hamiltonian is a function of these duality covariant variables: they transform into each other under symplectic rotations in a way that is independent of the details of the Hamiltonian. In fact, for a theory without higher derivatives, $\boldsymbol{A}$ and $\boldsymbol{D}$ are the canonical variables.

Electric/magnetic duality transformations thus act as canonical transformations and the Hamiltonian will usually decompose into a number of different functions that transform consistently under them. When the canonical transformations constitute an invariance of the system then these functions will be invariant. As it turns out, the Hesse potential of real special geometry is the direct analogue of the Hamiltonian. Rather than depending on the fields $X^{I}$, it depends on canonical variables $\phi^{I}$ and $\chi_{I}$. As we shall see, these can again be combined into complex variables in a way that involves the classical period vector associated with the function $F^{(0)}(X)$. The duality covariant variables $\left(\phi^{I}, \chi_{J}\right)$ transform under the same duality transformations as the classical period vector.

The Hesse potential is related to the function $F\left(X, W^{2}\right)$ via a Legendre transform, and thus contains the same information as the effective action. In principle, other relevant quantities that are related to the underlying Calabi-Yau moduli space, such as the topological string free energy, can be characterized by functions of $\left(\phi^{I}, \chi_{J}\right)$. Precisely as the Hamiltonian discussed above, the Hesse potential decomposes into different functions that all transform consistently under duality. The central conjecture of this paper is that the topological string should coincide with (part of) the Hesse potential, as this is the only way to explain why it can reproduce (part of) the effective action. To identify this particular function we will first consider what happens when the effective action is purely Wilsonian. As it turns out there is just one function belonging to the Hesse potential that is 'almost harmonic', where the meaning and implication of the term 'almost harmonic' will be explained in due course. The Hesse potential is nevertheless harmonic in terms of the (holomorphic) function that encodes the Wilsonian action. Subsequently it is demonstrated that, upon relaxing the harmonicity constraint on the function that encodes the effective action, the resulting 'almost harmonic' contribution to the Hesse potential satisfies the same holomorphic anomaly equation that is known from the topological string.

The paper is organized as follows. In section 2 we characterize possible non-holomorphic deformations of special geometry in the context of the effective action based on a theorem that is presented in appendix A. Subsequently we introduce the formulation of real special geometry in terms of the Hesse potential, which transforms as a function under symplectic rotations of its real variables, and derive a number of results that are important 
for what follows in subsequent sections. Section 3 is devoted to evaluating the Hesse potential in terms of complex duality covariant variables by carrying out the Legendre transform by iteration to fourth order, which is sufficient to appreciate its general structure. At this stage the non-holomorphic contributions can be understood in the context of a diagrammatic representation of the Hesse potential as a sum over connected tree graphs. The Hesse potential decomposes into an infinite number of terms, which arrange themselves into an infinite set of functions, all transforming consistently under electric/magnetic duality. Some of the expressions for these terms are collected in appendix B up to the corresponding order in the iteration. When the corresponding effective action is characterized in terms of a holomorphic function, precisely one of the functions contributing to the Hesse potential becomes 'almost harmonic' in the moduli. This function is thus the only possible candidate for a topological string free energy, and in section 4 we demonstrate that it indeed satisfies a holomorphic anomaly equation which partially coincides with the holomorphic anomaly equation known for the topological string [11].

Subsequently we relax the harmonicity restriction on the effective action by allowing a specific non-holomorphic term that transforms as a symplectic function up to a term that is harmonic. Introducing such a term induces quite a large variety of additional contributions to the Hesse potential that leave its characteristic properties intact, but the candidate function for the topological string free energy now satisfies the full holomorphic anomaly equation. Hence this function has now all the prerequisites for representing the generating function of the genus- $g$ free energies of the topological string and we explicitly demonstrate this up to $g \leq 3$. The calculation for $g=3$ is rather involved and it is described in section 5. We should stress here that the logic of our calculations is rather different from the one that is often followed for the topological string, where the non-holomorphic corrections are found by integrating the anomaly equation [11, 26, 27], with the holomorphic contributions playing the role of generalized integration 'constants'. In this paper we construct the Hesse potential starting from holomorphic functions, which, in order to ensure that they transform consistently under duality transformations, will necessarily contain non-holomorphic contributions. These non-holomorphic contributions then turn out to satisfy the holomorphic anomaly equation. In this way it is obvious that the holomorphic anomaly arises due to an incompatibility between duality covariance and holomorphicity. On the other hand we demonstrate that the function that encodes the effective action, which is holomorphic in the Wilsonian limit, must also contain corresponding non-holomorphic corrections of a specific form, which we evaluate order-by-order by iteration. All these results are established in the context of a generic special-Kähler space, but we do not wish to imply that in all these cases an actual topological string realization will exist.

A summary and a discussion of the results is presented in section 6. Here we also present a comparison of our present results with previous work [15, 16] on the FHSV model [28]. Furthermore we briefly discuss some of the consequences of the results of this paper for BPS black hole entropy, especially in connection with its conjectured relation to the topological string [29].

There are five appendices. The first appendix A establishes the consistency of special geometry under non-holomorphic deformations. The second appendix B lists a number of 
symplectic functions that emerge when evaluating the Hesse potential by iteration. Appendix $\mathrm{C}$ lists some intermediate results that are relevant for the third-order calculation described in section 5. The explicit expressions for the twisted string free energies $F^{(g)}$ of genus $g \leq 3$ are presented in appendix D based on the construction presented in this paper. Finally in appendix E we give further details about the comparison of the present results to earlier results obtained for the FHSV model.

\section{$2 \quad$ Real and deformed special geometry}

In the previous section we introduced holomorphic functions that encode either the Wilsonian action or the topological string free energy, as well as a real function known as the Hesse potential. While the first two are initially holomorphic, they eventually acquire nonholomorphic terms caused by the underlying physics. In the Hesse potential there seems no immediate obstacle to include such modifications as it is initially defined in terms of real variables. Let us now reiterate some of the distinctive features of these three structures and clarify the relevant issues.

The topological string free energy is a function of the Calabi-Yau moduli which are subject to dualities related to the homology group of the underlying holomorphic threeform. As explained in section 1, these moduli are associated with a holomorphic function $F^{(0)}(X)$, which also encodes a corresponding vector multiplet Lagrangian with at most two space-time derivatives coupled to supergravity. The dualities of this Lagrangian are generated by certain electric/magnetic dualities and they are related to the (discrete) homology group associated with the Calabi-Yau periods. When deforming the supergravity, for instance by introducing couplings to the square of the Weyl multiplet as specified in (1.4), the duality transformations of the moduli $X^{I}$ will change their form, whereas the variables $X^{I}$ in the topological string will still be associated with $F^{(0)}(X)$. Therefore, as explained in the previous section, the supergravity definition and the topological string definition of the variables $X^{I}$ will no longer be the same, and correspondingly the genus- $g$ free energies cannot be identical to the higher-derivative supergravity couplings.

The topological string free energy contains non-holomorphic corrections related to the pinchings of cycles in the underlying Riemann surfaces. These corrections should presumably be related to the non-holomorphic contributions to the function $F\left(X, W^{2}\right)$ which are induced by the integration over massless modes, in view of the fact that the two quantities are known to describe the same (on-shell) string amplitudes [11, 17]. Irrespective of this relationship the situation regarding the non-holomorphic corrections to the function $F\left(X, W^{2}\right)$ is subtle. Integrating out the massless modes leads to interactions that are non-local in generic space-times and it is not known what the precise dictionary is between non-holomorphic terms in the function $F$ and the non-local terms in the Lagrangian. In the supergravity context, non-holomorphic corrections are most likely related to chiral anomalies associated with the U(1) local symmetry that is an essential part of the superconformal multiplet calculus. These anomalies are cancelled by the non-holomorphic terms that emerge in the effective action. Such a phenomenon has been clarified in [30] for 
a number of situations. Another relevant observation is that non-holomorphic corrections are often required in order to have an exact duality invariance.

The variables $X^{I}$ and $W^{2}$ are only projectively defined, so that physically relevant results should not depend on uniform rescalings by a complex number. Hence we can replace the $X^{I}$ by uniformly rescaled variables $Y^{I}$ that differ by a uniform multiplicative complex factor or field according to a prescription that may depend on the application that is being considered. Likewise one must also rescale the expression for $W^{2}$ by the square of the same factor as for the $X^{I}$. The resulting expression is usually denoted by $\Upsilon$. However, in what follows we will regard $\Upsilon$ as one of the generalized coupling constants that may play a role. As it turns out it is not necessary to refer explicitly to such coupling constants, so that we will suppress them henceforth.

The information encoded in $F\left(X, W^{2}\right)$ can also be encoded in the context of real special geometry where the relevant quantity is the Hesse potential. As was already argued in the introductory section the Hesse potential represents the Hamiltonian form of the Wilsonian action and depends on real duality-covariant variables denoted by $\phi^{I}$ and $\chi_{I}$. They can be defined by

$$
\phi^{I}=Y^{I}+\bar{Y}^{\bar{I}}, \quad \chi_{I}=F_{I}+\bar{F}_{I} .
$$

Note that the replacement of the original variables $X^{I}$ by $Y^{I}$ is now relevant, as it would not make sense to consider linear combinations of the original variables $X^{I}$ and their complex conjugates in view of the fact that they are projectively defined. ${ }^{5}$ As it turns out, nonholomorphic corrections can be encoded in a real function $\Omega(Y, \bar{Y})$, which is incorporated into the function $F$ in the following way [32],

$$
F(Y, \bar{Y})=F^{(0)}(Y)+2 \mathrm{i} \Omega(Y, \bar{Y}),
$$

where $F^{(0)}(Y)$ is holomorphic and homogeneous of second degree. Note that the decomposition (2.2) is subject to the equivalence transformation,

$$
F^{(0)}(Y) \rightarrow F^{(0)}(Y)+g(Y), \quad \Omega(Y, \bar{Y}) \rightarrow \Omega(Y, \bar{Y})-\operatorname{Im} g(Y),
$$

which amounts to a shift of $F(Y, \bar{Y})$ by an anti-holomorphic function: $F(Y, \bar{Y}) \rightarrow F(Y, \bar{Y})+$ $\bar{g}(\bar{Y})$. This change does not affect the period vector $\left(Y^{I}, F_{I}\right)$, which only involves holomorphic derivatives, which is the underlying reason for this equivalence. When the function $\Omega$ is harmonic, i.e., when it can be written as the sum of a holomorphic and an antiholomorphic function, then one may simply absorb the holomorphic part into the first term according to (2.3). We usually refer to $F^{(0)}(Y)$ as the classical contribution, because it refers to the part of the Lagrangian that is quadratic in space-time derivatives. In that case only the function $\Omega$ will depend on possible deformation parameters such as $\Upsilon$ and $\bar{\Upsilon}$ and it may contain harmonic and non-harmonic contributions. The ansatz (2.2) may seem somewhat ad hoc, but in fact it can be derived in a much more general context as

\footnotetext{
${ }^{5}$ The same strategy was followed previously in the study of BPS black holes (see, e.g. [31]). The same comment applies to the holomorphic derivatives $F_{I}$. Note that $\bar{F}_{I}$ equals the derivative of $\bar{F}$ with respect to $\bar{Y}^{I}$. At this point we refrain from distinguishing holomorphic and anti-holomorphic derivatives, $\partial / \partial Y^{I}$ and $\partial / \partial \bar{Y}^{I}$, by the use of different types of indices.
} 
proven in the theorem presented in appendix A, which makes use of the analogue of the Hesse potential. The first indication for these results came from the study of BPS black hole entropy [15, 16, 22, 32, 33].

The new variables (2.1) have the virtue of transforming linearly under duality by real symplectic rotations. At this point it is convenient to define a quantity $\mathcal{H}$ of $\phi^{I}$ and $\chi_{I}$, which contains the same information as the $F(Y, \bar{Y})$ but transforms as a function under the duality transformations. This quantity is the Hesse potential. It is a generalization of the Hesse potential that was defined in the context of real special geometry [23-25] and follows from the Legendre transform of $4\left(\operatorname{Im} F^{(0)}+\Omega\right)$ with respect to the imaginary part of $Y^{I}$,

$$
\mathcal{H}(\phi, \chi)=4\left[\operatorname{Im} F^{(0)}(Y)+\Omega(Y, \bar{Y})\right]+\mathrm{i} \chi_{I}\left(Y^{I}-\bar{Y}^{I}\right) .
$$

Its generic variation satisfies

$$
\delta \mathcal{H}=-\mathrm{i}\left(F_{I}-\bar{F}_{I}\right) \delta \phi^{I}+\mathrm{i}\left(Y^{I}-\bar{Y}^{I}\right) \delta \chi_{I},
$$

where $F_{I}$ refers to the holomorphic derivative of $(2.2)$, which confirms that $\mathcal{H}$ is indeed a function of the duality-covariant variables $\left(\phi^{I}, \chi_{I}\right)$. The theorem of appendix A demonstrates that many of the special geometry properties remain valid under non-holomorphic deformations. This result had already been indicated by earlier work on this subject in [15].

The classical function $F^{(0)}$ is assumed to be holomorphic and homogeneous of second degree in $Y^{I}$. In the remainder of the section we summarize some results for such a function with respect to its behaviour under electric/magnetic duality that are needed in the next section. The electric/magnetic dualities are defined by $\operatorname{Sp}(2 n+2, \mathbb{R})$ rotations of the period vector $\left(Y^{I}, F_{I}\right)$, defined in the usual way,

$$
\begin{gathered}
Y^{I} \rightarrow \tilde{Y}^{I}=U_{J}^{I} Y^{J}+Z^{I J} F_{J}, \\
F_{I} \rightarrow \tilde{F}_{I}=V_{I}{ }^{J} F_{J}+W_{I J} Y^{J},
\end{gathered}
$$

where $U, V, Z$ and $W$ are the $(n+1) \times(n+1)$ real submatrices that constitute an element of $\operatorname{Sp}(2 n+2, \mathbb{R})$. Applying these transformations to the case where $F=F^{(0)}$, so that we are dealing with a homogeneous and holomorphic function, it follows that $\tilde{F}^{0)}{ }_{I}$ can be expressed as the holomorphic derivative of a new holomorphic function, $\tilde{F}^{(0)}(\tilde{Y})$, with the latter equal to

$$
\begin{aligned}
\tilde{F}^{(0)}(\tilde{Y})= & F^{(0)}(Y)-\frac{1}{2} Y^{I} F^{(0)}(Y)+\frac{1}{2}\left(U^{\mathrm{T}} W\right)_{I J} Y^{I} Y^{J} \\
& +\frac{1}{2}\left(U^{\mathrm{T}} V+W^{\mathrm{T}} Z\right)_{I}^{J} Y^{I} F^{(0)}{ }_{J}(Y)+\frac{1}{2}\left(Z^{\mathrm{T}} V\right)^{I J} F^{(0)}{ }_{I}(Y) F^{(0)}{ }_{J}(Y)
\end{aligned}
$$

which, in general, is difficult to solve explicitly. Note that, when the function is not homogeneous, there are integration constants corresponding to either a constant or terms proportional to the $\tilde{Y}^{I}$. In the presence of non-holomorphic terms the proof of existence of a new function is much more complicated, but the arguments, presented in a more generic context in appendix A, indicate that this is indeed the case, although no explicit expression has been given in the general case in analogy to (2.7). 
Finally we present the transformation rules of the first multiple derivatives of the function $F^{(0)}$ under the dualities (2.6),

$$
\begin{aligned}
& \tilde{F}^{(0)}{ }_{I J}=\left(V_{I}^{L} F^{(0)}{ }_{L K}+W_{I K}\right)\left[\mathcal{S}_{0}^{-1}\right]^{K}{ }_{J}, \\
& \tilde{F}^{(0)}{ }_{I J K}=\left[\mathcal{S}_{0}^{-1}\right]^{L}{ }_{I}\left[\mathcal{S}_{0}^{-1}\right]^{M}{ }_{J}\left[\mathcal{S}_{0}^{-1}\right]^{N}{ }_{K} F^{(0)}{ }_{L M N}, \\
& \tilde{F}^{(0)}{ }_{I J K L}=\left[\mathcal{S}_{0}^{-1}\right]^{\left(M_{I}\right.}\left[\mathcal{S}_{0}^{-1}\right]^{N}{ }_{J}\left[\mathcal{S}_{0}^{-1}\right]^{P}{ }_{K}\left[\mathcal{S}_{0}^{-1}\right]^{Q)}{ }_{L} \\
& \times\left[F^{(0)}{ }_{M N P Q}-3 F^{(0)} \bullet M N \mathcal{Z}_{0} \bullet F^{(0)}{ }_{P Q} \bullet\right], \\
& \tilde{F}^{(0)}{ }_{I J K L M}=\left[\mathcal{S}_{0}^{-1}\right]^{\left({ }^{N}\right.}{ }_{I}\left[\mathcal{S}_{0}^{-1}\right]^{P}{ }_{J}\left[\mathcal{S}_{0}^{-1}\right]^{Q}{ }_{K}\left[\mathcal{S}_{0}^{-1}\right]^{R}{ }_{L}\left[\mathcal{S}_{0}^{-1}\right]^{S)}{ }_{M} \\
& \times\left[F^{(0)}{ }_{N P Q R S}-10 F^{(0)} \bullet N P Q \mathcal{Z}_{0}{ }^{\bullet \bullet} F^{(0)} R S \bullet\right. \\
& \left.+15 F^{(0)} \bullet N P \mathcal{Z}_{0}^{\bullet \bullet} F^{(0)} \bullet Q \mathcal{Z}_{0}^{\bullet \bullet} F^{(0)} R S \bullet\right], \\
& \tilde{F}^{(0)}{ }_{I J K L M N}=\left[\mathcal{S}_{0}^{-1}\right]^{\left(P_{I}\right.}\left[\mathcal{S}_{0}^{-1}\right]^{Q}{ }_{J}\left[\mathcal{S}_{0}^{-1}\right]^{R}{ }_{K}\left[\mathcal{S}_{0}^{-1}\right]^{S}{ }_{L}\left[\mathcal{S}_{0}^{-1}\right]^{T}{ }_{M}\left[\mathcal{S}_{0}^{-1}\right]^{U)}{ }_{N} \\
& \times\left[F^{(0)} P Q R S T U-15 F^{(0)} \bullet P Q R S \mathcal{Z}_{0}^{\bullet \bullet} F^{(0)} T U \bullet\right. \\
& -10 F^{(0)} \bullet P Q R \mathcal{Z}_{0}^{\bullet \bullet} F^{(0)} S T U \bullet \\
& +60 F^{(0)} \bullet P Q R \mathcal{Z}_{0} \bullet F^{(0)} \bullet \mathcal{Z}_{0} \mathcal{Z}^{\bullet \bullet} F^{(0)} T U \bullet \\
& +45 F^{(0)} \bullet P Q \mathcal{Z}_{0} \bullet F^{(0)} \bullet R S \mathcal{Z}_{0} \bullet F^{(0)} T U \bullet \\
& -90 F^{(0)} \bullet P Q \mathcal{Z}_{0} \bullet F^{(0)} \bullet R \mathcal{Z}_{0}^{\bullet \bullet} F^{(0)} \bullet \mathcal{Z}_{0} \bullet F^{(0)} T U \bullet \\
& -15 F^{(0)} X Y Z \\
& \times\left[\mathcal{Z}_{0}{ }^{X \bullet} F_{P Q}^{(0)}{ }_{P Q} \bullet\left[\mathcal{Z}_{0}^{Y \bullet} F^{(0)} R S \bullet\right]\left[\mathcal{Z}_{0}^{Z \bullet} F^{(0)} T U \bullet\right]\right],
\end{aligned}
$$

which can be obtained by repeated differentiation of the basic equation (2.6) or (2.7). Note that for clarity we have occasionally replaced indices by bullets in cases where the index contractions are unambiguous. In the above formulae the bullets are indices that are simply contracted with the nearest neighbour bullet as a string. Furthermore we have made use of the following definitions,

$$
\begin{aligned}
\mathcal{S}_{0}{ }^{I}{ }_{J}(Y) & =\frac{\partial \tilde{Y}^{I}}{\partial Y^{J}}=U^{I}{ }_{J}+Z^{I K} F_{K J}^{(0)}(Y), \\
\mathcal{Z}_{0}^{I J}(Y) & =\left[\mathcal{S}_{0}^{-1}\right]^{I}{ }_{K} Z^{K J} .
\end{aligned}
$$

Because the matrices $U$ and $Z$ are submatrices of a $(2 n+2)$-dimensional symplectic matrix, it follows that $\mathcal{Z}_{0}^{I J}$ is symmetric in $(I, J)$. It also follows that

$$
\begin{aligned}
\delta\left[\mathcal{S}_{0}^{-1}\right]^{I}{ }_{J} & =-\mathcal{Z}_{0}{ }^{I K} \delta F^{(0)}{ }_{K L}\left[\mathcal{S}_{0}^{-1}\right]^{L}{ }_{J} \\
\delta \mathcal{Z}_{0}{ }^{I J} & =-\mathcal{Z}_{0}{ }^{I K} \delta F^{(0)}{ }_{K L} \mathcal{Z}_{0}{ }^{L J}
\end{aligned}
$$

Defining

$$
N_{I J}^{(0)}(Y, \bar{Y})=2 \operatorname{Im}\left[F^{(0)}{ }_{I J}(Y)\right]
$$

we derive the following expression for the behaviour of its inverse $N^{(0) I J}$ under duality transformations,

$$
\tilde{N}^{(0) I J}=\mathcal{S}_{0}{ }_{K} \overline{\mathcal{S}}_{0}{ }_{L}{ } N^{(0) K L} .
$$


Using the identity $\left[\mathcal{S}_{0}^{-1}\right]^{I}{ }_{K}\left[\overline{\mathcal{S}}_{0}\right]^{K}{ }_{J}=\delta^{I}{ }_{J}-\mathrm{i} \mathcal{Z}_{0}^{I K} N^{(0)}{ }_{K J}$, it follows that $(2.12)$ can also be written as,

$$
\tilde{N}^{(0) I J}=\mathcal{S}_{0}{ }^{I}{ }_{K} \mathcal{S}_{0}{ }^{J}{ }_{L}\left[N^{(0) K L}-\mathrm{i} \mathcal{Z}_{0}^{K L}\right]=\overline{\mathcal{S}}_{0}{ }^{I}{ }_{K} \overline{\mathcal{S}}_{0}{ }^{J}{ }_{L}\left[N^{(0) K L}+\mathrm{i} \overline{\mathcal{Z}}_{0}^{K L}\right] .
$$

These identities will be relevant later on. Incidentally, from the results presented above one can straightforwardly construct tensors that transform covariantly under the symplectic transformations, such as

$$
\left.C_{I J K L}=F^{(0)}{ }_{I J K L}+3 \mathrm{i} N^{(0) M N} F_{M(I J}^{(0)} F^{(0)} K L\right) N .
$$

However, these tensors are not purely holomorphic in view of the appearance of the matrix $N^{(0) I J}$. We will encounter such 'almost holomorphic' covariant functions throughout this paper.

Note that in the next section we will introduce different complex variables denoted by $\mathcal{Y}^{I}$. Since we will treat $\Omega$ as a perturbation of the classical function $F^{(0)}$, the full function $F$ will no longer play a role in the various formulae. Therefore, in due course, we will simply suppress all sub- and superscripts ' 0 ' referring to the lowest-order quantities $F^{(0)}, \mathcal{S}_{0}{ }^{I}{ }_{J}, \mathcal{Z}_{0}^{I J}$, and $N^{(0) I J}$. Note also that we are only distinguishing holomorphic and anti-holomorphic derivatives, $I, J, \ldots$ and $\bar{I}, \bar{J}, \ldots$ with a bar when we are dealing with a real quantity. For instance, $\overline{\mathcal{S}}_{0}{ }_{K}$ is anti-holomorphic, so we will just keep generic indices $J, K$ (rather than $\bar{J}, \bar{K}$ ), while $\Omega_{I}$ and $\Omega_{\bar{I}}$ need a holomorphic or anti-holomorphic index to distinguish between the derivative with respect to $Y^{I}$ and $\bar{Y}^{I}$. The reason for this convention is that we will often not have the situation where holomorphic and anti-holomorphic indices are contracted consistently.

\section{The generic structure of the Hesse potential}

In this section we study the generic structure of the Hesse potential, which requires to carry out a Legendre transform. In practice this can only be done by iteration. The results will then take the form of an infinite power series in terms of $\Omega$ and its derivatives. We will explicitly evaluate the first terms in this expansion up to order $\Omega^{5}$, which we expect to suffice for uncovering the general structure of the full expression. The homogeneous and holomorphic function $F^{(0)}(Y)$ will not be subject to further restrictions and will thus encode a generic special-Kähler space, while $\Omega$ will be an arbitrary function of $Y$ and $\bar{Y}$. The actual calculations are rather laborious although in principle straightforward; we have relegated some relevant material to several appendices. Similar calculations have been performed for more specific cases some time ago $[15,16]$. For instance, the first two terms to quadratic order in $\Upsilon, \bar{\Upsilon}$ have been determined for the FHSV model [28]. As it turned out some of these terms were consistent with known results obtained from the topological string by integration of the holomorphic anomaly equation [27], but the Hesse potential contained additional terms at this order that were separately S- and T-duality invariant but did not have an interpretation in the topological string context. In this paper we will investigate the generic structure of the Hesse potential and clarify these partial results. We will return to a discussion of the results for the FHSV model in section 6 . 
To carry out the Legendre transform by iteration we first choose convenient variables. Originally the Hesse potential was defined in terms of the variables $\left(\phi^{I}, \chi_{I}\right)$ of real special geometry, whose definition involves the full effective action. It is, however, more convenient to convert them again to complex variables, subsequently denoted by $\mathcal{Y}^{I}$, which coincide precisely with the fields $Y^{I}$ that one would obtain from $\left(\phi^{I}, \chi_{I}\right)$ upon using just the lowestorder holomorphic function $F^{(0)}$. The identification proceeds as follows [16],

$$
\begin{aligned}
2 \operatorname{Re} Y^{I} & =\phi^{I}=2 \operatorname{Re} \mathcal{Y}^{I}, \\
2 \operatorname{Re} F_{I}(Y, \bar{Y}) & =\chi_{I}=2 \operatorname{Re} F^{(0)}{ }_{I}(\mathcal{Y}) .
\end{aligned}
$$

Since the relation between the variables $\mathcal{Y}^{I}$ and the real variables $\left(\phi^{I}, \chi_{I}\right)$ involves only $F^{(0)}$, their duality transformations will be directly related. Consequently we will refer to the variables $\mathcal{Y}^{I}$ as duality covariant variables. Under duality the variables $\mathcal{Y}^{I}$ transform according to,

$$
\tilde{\mathcal{Y}}^{I}=U^{I}{ }_{J} \mathcal{Y}^{J}+Z^{I J} F^{(0)}(\mathcal{Y})=\mathcal{S}^{I}{ }_{J}(\mathcal{Y}) \mathcal{Y}^{J},
$$

where we used the homogeneity of $F^{(0)}(\mathcal{Y})$ as well as the definition of $\mathcal{S}^{I} J$ given in (2.9), except that the expression is now written in terms of the new variables $\mathcal{Y}^{I}$. Furthermore we have dropped the subscript by the replacement $\mathcal{S}_{0} \rightarrow \mathcal{S}$, as we had already indicated at the end of section 2.

At the classical level, where $\Omega=0$, we obviously have $\mathcal{Y}^{I}=Y^{I}$, but in higher orders the relation between these moduli is complicated and will involve $\Omega$. Let us therefore write $\mathcal{Y}^{I}=Y^{I}+\Delta Y^{I}$, where $\Delta Y^{I}$ is purely imaginary, and $F=F^{(0)}+2 \mathrm{i} \Omega$, so that we can express (3.1) in terms of $F^{(0)}, \Omega, \mathcal{Y}^{I}$ and $\Delta Y^{I}$. Because the equations will no longer involve $F$, we will henceforth drop the index ' 0 ' on $F^{(0)}$, as mentioned earlier. Consequently all the derivatives of $F$ will be holomorphic. The equations (3.1) can then be written as,

$$
\begin{aligned}
& F_{I}(\mathcal{Y}-\Delta Y)+\bar{F}_{I}(\overline{\mathcal{Y}}+\Delta Y)-F_{I}(\mathcal{Y})-\bar{F}_{I}(\overline{\mathcal{Y}}) \\
= & -2 \mathrm{i}\left[\Omega_{I}(\mathcal{Y}-\Delta Y, \overline{\mathcal{Y}}+\Delta Y)-\Omega_{\bar{I}}(\mathcal{Y}-\Delta Y, \overline{\mathcal{Y}}+\Delta Y)\right],
\end{aligned}
$$

where we made use of (2.2). Upon Taylor expanding, this equation will lead to an infinite power series in $\Delta Y^{I}$, which we can solve by iteration. Retaining only the term of first order in $\Delta Y^{I}$ shows that it is proportional to first derivatives of $\Omega$. Proceeding to higher orders will then lead to an expression for $\Delta Y^{I}$ involving increasing powers of $\Omega$ and $F$ and their derivatives taken at $Y^{I}=\mathcal{Y}^{I}$. Up to fourth order in $\Omega$ this iteration gives the following expression for $\Delta Y^{I}$,

$$
\begin{aligned}
\Delta Y^{I} \approx & 2\left(\Omega^{I}-\Omega^{\bar{I}}\right) \\
& -2 \mathrm{i}(F+\bar{F})^{I J K}\left(\Omega_{J}-\Omega_{\bar{J}}\right)\left(\Omega_{K}-\Omega_{\bar{K}}\right)-8 \operatorname{Re}\left(\Omega^{I J}-\Omega^{I \bar{J}}\right)\left(\Omega_{J}-\Omega_{\bar{J}}\right) \\
& +\frac{4}{3} \mathrm{i}\left[(F-\bar{F})^{I J K L}+3 \mathrm{i}(F+\bar{F})^{I J M}(F+\bar{F})_{M}{ }^{K L}\right] \\
& \quad \times\left(\Omega_{J}-\Omega_{\bar{J}}\right)\left(\Omega_{K}-\Omega_{\bar{K}}\right)\left(\Omega_{L}-\Omega_{\bar{L}}\right) \\
& +8 \mathrm{i}\left[2(F+\bar{F})^{I J}{ }_{K} \operatorname{Re}\left(\Omega^{K L}-\Omega^{K \bar{L}}\right)+\operatorname{Re}\left(\Omega^{I K}-\Omega^{I \bar{K}}\right)(F+\bar{F})_{K}{ }^{J L}\right] \\
& \times\left(\Omega_{J}-\Omega_{\bar{J}}\right)\left(\Omega_{L}-\Omega_{\bar{L}}\right)
\end{aligned}
$$




$$
\begin{aligned}
& +32 \operatorname{Re}\left(\Omega^{I J}-\Omega^{I \bar{J}}\right) \operatorname{Re}\left(\Omega_{J K}-\Omega_{J \bar{K}}\right)\left(\Omega^{K}-\Omega^{\bar{K}}\right) \\
& +8 \mathrm{i} \operatorname{Im}\left(\Omega^{I J K}-2 \Omega^{I J \bar{K}}+\Omega^{I \bar{J} \bar{K}}\right)\left(\Omega_{J}-\Omega_{\bar{J}}\right)\left(\Omega_{K}-\Omega_{\bar{K}}\right)+\mathcal{O}\left(\Omega^{4}\right) .
\end{aligned}
$$

Here indices have been raised by making use of $N^{(0) I J}$, which was already defined in (2.11), where, for consistency, we will henceforth change notation and refer to $N^{(0) I J}$ by $N^{I J}$. Here we stress once more that all the derivatives of $F$ and $\Omega$ are taken at $Y^{I}=\mathcal{Y}^{I}$ and $\bar{Y}^{I}=\overline{\mathcal{Y}}^{I}$.

Using the same notation we obtain the following expression for the Hesse potential (2.4),

$$
\begin{aligned}
\mathcal{H}(\mathcal{Y}, \overline{\mathcal{Y}})= & -\mathrm{i}\left[\overline{\mathcal{Y}}^{I} F_{I}(\mathcal{Y})-\mathcal{Y}^{I} \bar{F}_{I}(\overline{\mathcal{Y}})\right]+4 \Omega(\mathcal{Y}, \overline{\mathcal{Y}}) \\
& -\mathrm{i}\left[\mathcal{Y}^{I}\left(F_{I}(Y)-F_{I}(\mathcal{Y})\right)+\Delta Y^{I} F_{I}(Y)-\text { h.c. }\right] \\
& +4\left[\Omega(Y, \bar{Y})-\Omega(\mathcal{Y}, \overline{\mathcal{Y}})+\Delta Y^{I}\left(\Omega_{I}(Y, \bar{Y})-\Omega_{\bar{I}}(Y, \bar{Y})\right)\right] .
\end{aligned}
$$

Here we made use of the homogeneity of $F(Y)$ and of (3.3). Again this result must be Taylor expanded upon writing $Y^{I}=\mathcal{Y}^{I}-\Delta Y^{I}$ and $\bar{Y}^{I}=\overline{\mathcal{Y}}^{I}+\Delta Y^{I}$. The last two lines of (3.5) then lead to a power series in $\Delta Y$, starting at second order in the $\Delta Y$,

$$
\begin{aligned}
\mathcal{H}(\mathcal{Y}, \overline{\mathcal{Y}}) \approx & -\mathrm{i}\left[\overline{\mathcal{Y}}^{I} F_{I}(\mathcal{Y})-\mathcal{Y}^{I} \bar{F}_{I}(\overline{\mathcal{Y}})\right]+4 \Omega(\mathcal{Y}, \overline{\mathcal{Y}}) \\
& -N_{I J} \Delta Y^{I} \Delta Y^{J}-\frac{2}{3} \mathrm{i}(F+\bar{F})_{I J K} \Delta Y^{I} \Delta Y^{J} \Delta Y^{K} \\
& -4 \operatorname{Re}\left(\Omega_{I J}-\Omega_{I \bar{J}}\right) \Delta Y^{I} \Delta Y^{J}+\frac{1}{4} \mathrm{i}(F-\bar{F})_{I J K L} \Delta Y^{I} \Delta Y^{J} \Delta Y^{K} \Delta Y^{L} \\
& +\frac{8}{3} \mathrm{i} \operatorname{Im}\left(\Omega_{I J K}-3 \Omega_{I J \bar{K}}\right) \Delta Y^{I} \Delta Y^{J} \Delta Y^{K}+\cdots
\end{aligned}
$$

Inserting the result of the iteration (3.4) into the expression above leads to the following expression for the Hesse potential, up to terms of order $\Omega^{5}$,

$$
\begin{aligned}
\mathcal{H}(\mathcal{Y}, \overline{\mathcal{Y}}) \approx & -\mathrm{i}\left[\overline{\mathcal{Y}}^{I} F_{I}(\mathcal{Y})-\mathcal{Y}^{I} \bar{F}_{I}(\overline{\mathcal{Y}})\right]+4 \Omega(\mathcal{Y}, \overline{\mathcal{Y}}) \\
- & 4 \hat{N}^{I J} z_{I} z_{J}+\frac{8}{3} \mathrm{i}(F+\bar{F})_{I J K} \hat{N}^{I L} \hat{N}^{J M} \hat{N}^{K N} z_{L} z_{M} z_{N} \\
- & \frac{4}{3} \mathrm{i}\left[(F-\bar{F})_{I J K L}+3 \mathrm{i}(F+\bar{F})_{I J R} \hat{N}^{R S}(F+\bar{F})_{S K L}\right] \\
& \quad \times \hat{N}^{I M} \hat{N}^{J N} \hat{N}^{K P} \hat{N}^{L Q} z_{M} z_{N} z_{P} z_{Q} \\
- & \frac{32}{3} \mathrm{i} \operatorname{Im}\left(\Omega_{I J K}-3 \Omega_{I J \bar{K}}\right) \hat{N}^{I L} \hat{N}^{J M} \hat{N}^{K N} z_{L} z_{M} z_{N}+\mathcal{O}\left(\Omega^{5}\right)
\end{aligned}
$$

where $z_{I}=\Omega_{I}-\Omega_{\bar{I}}$, and where $\hat{N}^{I J}$ is the inverse of the real, symmetric matrix $\hat{N}_{I J}$, defined by

$$
\hat{N}_{I J}=N_{I J}+4 \operatorname{Re}\left(\Omega_{I J}-\Omega_{I \bar{J}}\right) .
$$

Upon expanding $\hat{N}^{I J}$ we straightforwardly determine the contributions to the Hesse po- 
tential up to fifth order in $\Omega$,

$$
\begin{aligned}
\mathcal{H}= & \left.\mathcal{H}\right|_{\Omega=0}+4 \Omega-4 N^{I J}\left(\Omega_{I} \Omega_{J}+\Omega_{\bar{I}} \Omega_{\bar{J}}\right)+8 N^{I J} \Omega_{I} \Omega_{\bar{J}} \\
& +16 \operatorname{Re}\left(\Omega_{I J}-\Omega_{I \bar{J}}\right) N^{I K} N^{J L}\left(\Omega_{K} \Omega_{L}+\Omega_{\bar{K}} \Omega_{\bar{L}}-2 \Omega_{K} \Omega_{\bar{L}}\right) \\
& -\frac{16}{3}(F+\bar{F})_{I J K} N^{I L} N^{J M} N^{K N} \operatorname{Im}\left(\Omega_{L} \Omega_{M} \Omega_{N}-3 \Omega_{L} \Omega_{M} \Omega_{\bar{N}}\right) \\
& -64 N^{I P} \operatorname{Re}\left(\Omega_{P Q}-\Omega_{P \bar{Q}}\right) N^{Q R} \operatorname{Re}\left(\Omega_{R K}-\Omega_{R \bar{K}}\right) N^{K J}\left(\Omega_{I} \Omega_{J}+\Omega_{\bar{I}} \Omega_{\bar{J}}-2 \Omega_{I} \Omega_{\bar{J}}\right) \\
& +64(F+\bar{F})_{I J K} N^{I L} N^{J M} N^{K P} \operatorname{Re}\left(\Omega_{P Q}-\Omega_{P \bar{Q}}\right) N^{Q N} \operatorname{Im}\left(\Omega_{L} \Omega_{M} \Omega_{N}-3 \Omega_{L} \Omega_{M} \Omega_{\bar{N}}\right) \\
& -\frac{8}{3} \mathrm{i}\left[(F-\bar{F})_{I J K L}+3 \mathrm{i}(F+\bar{F})_{R(I J} N^{R S}(F+\bar{F})_{K L) S}\right] N^{I M} N^{J N} N^{K P} N^{L Q} \\
& \times \operatorname{Re}\left(\Omega_{M} \Omega_{N} \Omega_{P} \Omega_{Q}-4 \Omega_{M} \Omega_{N} \Omega_{P} \Omega_{\bar{Q}}+3 \Omega_{M} \Omega_{N} \Omega_{\bar{P}} \Omega_{\bar{Q}}\right) \\
& +\frac{64}{3} \operatorname{Im}\left(\Omega_{I J K}-3 \Omega_{I J \bar{K}}\right) N^{I L} N^{J M} N^{K N} \operatorname{Im}\left(\Omega_{L} \Omega_{M} \Omega_{N}-3 \Omega_{L} \Omega_{M} \Omega_{\bar{N}}\right)+\mathcal{O}\left(\Omega^{5}\right) .
\end{aligned}
$$

We stress once more that all the quantities in (3.9) are taken at $Y^{I}=\mathcal{Y}^{I}$.

It is clear that the Hesse potential takes a complicated form, but we note two systematic features. First of all it turns out that the expression (3.9) can be understood diagrammatically. To appreciate this, let us return to the definition (2.4) of the Hesse potential and rewrite it in a different form,

$$
\begin{aligned}
\mathcal{H}(\phi, \chi)= & 4[\operatorname{Im} F(Y)+\Omega(Y, \bar{Y})]+\mathrm{i} \chi_{I}\left(Y^{I}-\bar{Y}^{I}\right) \\
= & -2 \mathrm{i} F(\mathcal{Y}-\Delta Y)+2 \mathrm{i} \bar{F}(\overline{\mathcal{Y}}+\Delta Y)+4 \Omega(\mathcal{Y}-\Delta Y, \overline{\mathcal{Y}}+\Delta Y) \\
& +\mathrm{i} \chi_{I}\left(\mathcal{Y}^{I}-\overline{\mathcal{Y}}^{I}\right)-2 \mathrm{i} \chi_{I} \Delta Y^{I},
\end{aligned}
$$

where the purely imaginary quantities $\Delta Y^{I}$ were introduced in the beginning of section 3 when defining the iteration procedure. We remind the reader that $\phi^{I}=2 \operatorname{Re} Y^{I}=2 \operatorname{Re} \mathcal{Y}^{I}$ and $\chi_{I}=2 \operatorname{Re} F_{I}(\mathcal{Y})$. Substituting these results we derive

$$
\begin{aligned}
\mathcal{H}(\mathcal{Y}, \overline{\mathcal{Y}})= & \left.\mathcal{H}(\mathcal{Y}, \overline{\mathcal{Y}})\right|_{\Omega=0}+N_{I J}(\mathcal{Y}, \overline{\mathcal{Y}}) \Delta Y^{I} \Delta Y^{J} \\
& +2 \mathrm{i} \sum_{n=3}^{\infty} \frac{1}{n !}\left[(-)^{n+1} F_{I_{1} \cdots I_{n}}(\mathcal{Y})+\bar{F}_{I_{1} \cdots I_{n}}(\overline{\mathcal{Y}})\right] \Delta Y_{1} \cdots \Delta Y_{n} \\
& +4 \Omega(\mathcal{Y}-\Delta Y, \overline{\mathcal{Y}}+\Delta Y) .
\end{aligned}
$$

It thus follows that $\mathcal{H}(\mathcal{Y}, \overline{\mathcal{Y}})-\left.\mathcal{H}(\mathcal{Y}, \overline{\mathcal{Y}})\right|_{\Omega=0}-4 \Omega(\mathcal{Y}, \overline{\mathcal{Y}})$ can be written as a series expansion in positive powers of $\Delta Y^{I}$. Integrating the exponential of this expression over the (purely imaginary) fluctuations $\Delta Y^{I}$, the result can be expressed as an infinite sum over Feynman diagrams in the standard way with propagators given by $N^{I J}$ and vertices by the derivatives of $F$ and $\bar{F}$ as well as of the holomorphic and anti-holomorphic derivatives of $\Omega$. Because the Legendre transformation performed above should correspond to the tree diagrams, it follows that $\mathcal{H}(\mathcal{Y}, \overline{\mathcal{Y}})-\left.\mathcal{H}(\mathcal{Y}, \overline{\mathcal{Y}})\right|_{\Omega=0}-4 \Omega(\mathcal{Y}, \overline{\mathcal{Y}})$ will comprise all the connected tree diagrams. In this way one can account for all the terms in (3.9), including their combinatorial factors. Note that the diagrams are not 1PI: by removing the propagator $N^{I J}$ 
each term in the expression will factorize into two terms! Here we should mention that diagrammatic techniques have repeatedly played a role in the analysis of the holomorphic anomaly equation and of the topological string (see e.g. [11, 26, 27]).

Another feature is based on the fact that (3.9) transforms as a function under duality transformations of the fields $\mathcal{Y}^{I}$, and so does the first term $\left.\mathcal{H}\right|_{\Omega=0}$. This observation enables one to determine how $\Omega$ will transform under dualities. Obviously the transformation behaviour of $\Omega$ must be non-trivial in view of the non-linear dependence on $\Omega$ of the Hesse potential. The evaluation of this transformation proceeds again by iteration.

To demonstrate the procedure, let us review the first few steps. In lowest order, $\Omega$ must transform as a function, which implies that

$$
\begin{aligned}
\tilde{\Omega}(\tilde{\mathcal{Y}}, \overline{\mathcal{Y}}) & =\Omega(\mathcal{Y}, \overline{\mathcal{Y}})+\mathcal{O}\left(\Omega^{2}\right), \\
\tilde{\Omega}_{I}(\tilde{\mathcal{Y}}, \overline{\mathcal{Y}}) & =\left[\mathcal{S}^{-1}\right]^{J}{ }_{I}(\mathcal{Y}) \Omega_{J}(\mathcal{Y}, \overline{\mathcal{Y}})+\mathcal{O}\left(\Omega^{2}\right),
\end{aligned}
$$

where the matrix $\mathcal{S}$ was already defined previously (note that we have suppressed the subscript ' 0 '). Applying this result to the first few terms of (3.9) and making use of the fact that $\mathcal{H}-\left.\mathcal{H}\right|_{\Omega=0}$ transforms as a function, one deduces the next-order result,

$$
\begin{aligned}
& \tilde{\Omega}-\tilde{N}^{I J}\left(\tilde{\Omega}_{I} \tilde{\Omega}_{J}+\tilde{\Omega}_{\bar{I}} \tilde{\Omega}_{\bar{J}}\right)+2 \tilde{N}^{I J} \tilde{\Omega}_{I} \tilde{\Omega}_{\bar{J}}+\mathcal{O}\left(\tilde{\Omega}^{3}\right) \\
= & \Omega-N^{I J}\left(\Omega_{I} \Omega_{J}+\Omega_{\bar{I}} \Omega_{\bar{J}}\right)+2 N^{I J} \Omega_{I} \Omega_{\bar{J}}+\mathcal{O}\left(\Omega^{3}\right),
\end{aligned}
$$

where on the left-hand side the functions depend on the transformed fields $\tilde{\mathcal{Y}}^{I}$, while on the right-hand side they depend on the original fields $\mathcal{Y}^{I}$.

Using the exact relations (2.9)-(2.13), suppressing also the subscript ' 0 ' in the symmetric matrix $\mathcal{Z}_{0}^{I J}$, one discovers that the first equation in (3.12) receives the following correction in second order in $\Omega$,

$$
\tilde{\Omega}(\tilde{\mathcal{Y}}, \overline{\tilde{\mathcal{Y}}})=\Omega-\mathrm{i}\left(\mathcal{Z}^{I J} \Omega_{I} \Omega_{J}-\overline{\mathcal{Z}}^{I J} \Omega_{\bar{I}} \Omega_{\bar{J}}\right)+\mathcal{O}\left(\Omega^{3}\right)
$$

which in turn gives rise to the following result for derivatives of $\Omega$,

$$
\begin{aligned}
& \tilde{\Omega}_{I}(\tilde{\mathcal{Y}}, \overline{\mathcal{Y}})= {\left[\mathcal{S}^{-1}\right]_{I}^{J}\left[\Omega_{J}+\mathrm{i} F_{J K L} \mathcal{Z}^{K M} \Omega_{M} \mathcal{Z}^{L N} \Omega_{N}-2 \mathrm{i} \Omega_{J K} \mathcal{Z}^{K L} \Omega_{L}+2 \mathrm{i} \Omega_{J \bar{K}} \overline{\mathcal{Z}}^{K L} \Omega_{\bar{L}}\right] } \\
&+\mathcal{O}\left(\Omega^{3}\right), \\
& \tilde{\Omega}_{I J}(\tilde{\mathcal{Y}}, \overline{\mathcal{Y}})= {\left[\mathcal{S}^{-1}\right]^{K}\left[\mathcal{S}^{-1}\right]^{L}{ }_{J}\left[\Omega_{K L}-F_{K L M} \mathcal{Z}^{M N} \Omega_{N}\right]+\mathcal{O}\left(\Omega^{2}\right), } \\
& \tilde{\Omega}_{I \bar{J}}(\tilde{\mathcal{Y}}, \overline{\mathcal{Y}})=\left[\mathcal{S}^{-1}\right]^{K}{ }_{I}\left[\overline{\mathcal{S}}^{-1}\right]^{\bar{L}}{ }_{\bar{J}} \Omega_{K \bar{L}}+\mathcal{O}\left(\Omega^{2}\right) .
\end{aligned}
$$

Here and henceforth we make frequent use of (2.10).

The iteration can be continued by including the terms of order $\Omega^{3}$, making use of (3.15) for derivatives of $\Omega$, to obtain the expression for $\tilde{\Omega}$ up to terms of order $\Omega^{4}$. In the next iterative step one then derives the effect of a duality transformation on $\Omega$ up to terms of order $\Omega^{5}$. Before presenting this result, we wish to observe that terms transforming as a proper function under duality, will not contribute to this result. This is precisely what happens to the term proportional to $N^{I J} \Omega_{I} \Omega_{\bar{J}}$ that appears in (3.9), which transforms as 
a function under symplectic transformations in this order of the iteration. Consequently this term does not contribute to (3.14). As it turns out an infinite set of contributions to the Hesse potential will be generated that transform separately as functions under duality. By separating those from (3.9), we do not change the transformation behaviour of $\Omega$ but we can extract certain functions from the Hesse potential in order to simplify its structure. However, these functions must be constructed also by iteration, order by order in $\Omega$.

We have evaluated this decomposition in terms of separate functions in detail, which leads to

$$
\begin{aligned}
\mathcal{H}= & \mathcal{H}^{(0)}+\mathcal{H}^{(1)}+\mathcal{H}^{(2)}+\left(\mathcal{H}_{1}^{(3)}+\mathcal{H}_{2}^{(3)}+\text { h.c. }\right)+\mathcal{H}_{3}^{(3)}+\mathcal{H}_{1}^{(4)}+\mathcal{H}_{2}^{(4)}+\mathcal{H}_{3}^{(4)} \\
& +\left(\mathcal{H}_{4}^{(4)}+\mathcal{H}_{5}^{(4)}+\mathcal{H}_{6}^{(4)}+\mathcal{H}_{7}^{(4)}+\mathcal{H}_{8}^{(4)}+\mathcal{H}_{9}^{(4)}+\text { h.c. }\right) \ldots
\end{aligned}
$$

where the $\mathcal{H}_{i}^{(a)}$ are certain expressions to be defined below, whose leading term is of order $\Omega^{a}$. For higher values of $a$ it turns out that there exists more than one function with the same value of $a$, and those will be labeled by $i=1,2, \ldots$. Of all the combinations $\mathcal{H}_{i}^{(a)}$ appearing in (3.16), $\mathcal{H}^{(1)}$ is the only one that contains $\Omega$, while all the other combinations contain derivatives of $\Omega$. Obviously, $\mathcal{H}^{(0)}$ equals,

$$
\mathcal{H}^{(0)}=-\mathrm{i}\left[\overline{\mathcal{Y}}^{I} F_{I}(\mathcal{Y})-\mathcal{Y}^{I} \bar{F}_{I}(\overline{\mathcal{Y}})\right],
$$

whereas $\mathcal{H}^{(1)}$ at this level of iteration is given by,

$$
\begin{aligned}
\mathcal{H}^{(1)}= & 4 \Omega-4 N^{I J}\left(\Omega_{I} \Omega_{J}+\Omega_{\bar{I}} \Omega_{\bar{J}}\right) \\
& +16 \operatorname{Re}\left[\Omega_{I J}(N \Omega)^{I}(N \Omega)^{J}\right]+16 \Omega_{I \bar{J}}(N \Omega)^{I}(N \bar{\Omega})^{J} \\
& -\frac{16}{3} \operatorname{Im}\left[F_{I J K}(N \Omega)^{I}(N \Omega)^{J}(N \Omega)^{K}\right] \\
& -\frac{4}{3} \mathrm{i}\left[\left(F_{I J K L}+3 \mathrm{i} F_{R(I J} N^{R S} F_{K L) S}\right)(N \Omega)^{I}(N \Omega)^{J}(N \Omega)^{K}(N \Omega)^{L}-\text { h.c. }\right] \\
& -\frac{16}{3}\left[\Omega_{I J K}(N \Omega)^{I}(N \Omega)^{J}(N \Omega)^{K}+\text { h.c. }\right] \\
& -16\left[\Omega_{I J \bar{K}}(N \Omega)^{I}(N \Omega)^{J}(N \bar{\Omega})^{K}+\text { h.c. }\right] \\
& -16 \mathrm{i}\left[F_{I J K} N^{K P} \Omega_{P Q}(N \Omega)^{I}(N \Omega)^{J}(N \Omega)^{Q}-\text { h.c. }\right] \\
& -16\left[(N \Omega)^{P} \Omega_{P Q} N^{Q R} \Omega_{R K}(N \Omega)^{K}+\text { h.c. }\right] \\
& -16\left[(N \Omega)^{P} \Omega_{P Q} N^{Q R} \Omega_{R \bar{K}}(N \bar{\Omega})^{K}\right. \\
& \left.\quad+N^{I P} \Omega_{P \bar{Q}} N^{Q R}\left(\Omega_{\bar{R} K}(N \Omega)^{K}+\Omega_{\bar{R} \bar{K}}(N \bar{\Omega})^{K}\right) \Omega_{I}+\text { h.c. }\right] \\
& -16 \mathrm{i}\left[F_{I J K} N^{K P} \Omega_{P \bar{Q}}(N \Omega)^{I}(N \Omega)^{J}(N \bar{\Omega})^{Q}-\text { h.c. }\right]+\mathcal{O}\left(\Omega^{5}\right) .
\end{aligned}
$$

Here we have used the notation $(N \Omega)^{I}=N^{I J} \Omega_{J},(N \bar{\Omega})^{I}=N^{I J} \Omega_{\bar{J}}$. Index symmetrizations, such as in $F_{R(I J} N^{R S} F_{K L) S}$, are always of strength one. For instance, in this example, where there are three independent combinations, one includes a factor $1 / 3$. The expressions for the higher-order functions $\mathcal{H}_{i}^{(a)}$ with $a=2,3,4$ are given in appendix B. These 
expressions have been obtained by requiring that they constitute functions under symplectic transformations, order by order in $\Omega$. There exist other expressions that do transform as functions under duality in this approximation, but which do not appear in $\mathcal{H}$. We have included two examples of such functions in appendix B, one of which will be relevant later on.

Because $\mathcal{H}^{(1)}$ transforms as a function under symplectic transformations, we can deduce the transformation behavior of $\Omega$ up to order $\Omega^{5}$ by generalizing (3.12) to higher orders. In this way one derives,

$$
\begin{aligned}
\tilde{\Omega}(\tilde{\mathcal{Y}}, \overline{\mathcal{Y}})= & =\mathrm{i}\left(\mathcal{Z}^{I J} \Omega_{I} \Omega_{J}-\overline{\mathcal{Z}}^{I J} \Omega_{\bar{I}} \Omega_{\bar{J}}\right) \\
+ & \frac{2}{3}\left(F_{I J K} \mathcal{Z}^{I L} \Omega_{L} \mathcal{Z}^{J M} \Omega_{M} \mathcal{Z}^{K N} \Omega_{N}+\text { h.c. }\right) \\
- & 2\left(\Omega_{I J} \mathcal{Z}^{I K} \Omega_{K} \mathcal{Z}^{J L} \Omega_{L}+\text { h.c. }\right)+4 \Omega_{I \bar{J}} \mathcal{Z}^{I K} \Omega_{K} \overline{\mathcal{Z}}^{J L} \Omega_{\bar{L}} \\
+ & -\frac{1}{3} \mathrm{i} F_{I J K L}(\mathcal{Z} \Omega)^{I}(\mathcal{Z} \Omega)^{J}(\mathcal{Z} \Omega)^{K}(\mathcal{Z} \Omega)^{L} \\
& +\frac{4}{3} \mathrm{i} \Omega_{I J K}(\mathcal{Z} \Omega)^{I}(\mathcal{Z} \Omega)^{J}(\mathcal{Z} \Omega)^{K} \\
& +\mathrm{i} F_{I J R} \mathcal{Z}^{R S} F_{S K L}(\mathcal{Z} \Omega)^{I}(\mathcal{Z} \Omega)^{J}(\mathcal{Z} \Omega)^{K}(\mathcal{Z} \Omega)^{L} \\
& -4 \mathrm{i} \Omega_{I J \bar{K}}(\mathcal{Z} \Omega)^{I}(\mathcal{Z} \Omega)^{J}(\overline{\mathcal{Z}} \bar{\Omega})^{\bar{K}} \\
& -4 \mathrm{i} F_{I J K} \mathcal{Z}^{K P} \Omega_{P Q}(\mathcal{Z} \Omega)^{I}(\mathcal{Z} \Omega)^{J}(\mathcal{Z} \Omega)^{Q} \\
& +4 \mathrm{i} F_{I J K} \mathcal{Z}^{K P} \Omega_{P \bar{Q}}(\mathcal{Z} \Omega)^{I}(\mathcal{Z} \Omega)^{J}(\overline{\mathcal{Z}} \bar{\Omega})^{\bar{Q}} \\
& +4 \mathrm{i}(\mathcal{Z} \Omega)^{P} \Omega_{P Q} \mathcal{Z}^{Q R}\left(\Omega_{R K}(\mathcal{Z} \Omega)^{K}-2 \Omega_{R \bar{K}}(\overline{\mathcal{Z}} \bar{\Omega})^{\bar{K}}\right) \\
& \left.-4 \mathrm{i}(\mathcal{Z} \Omega)^{P} \Omega_{P \bar{Q}} \overline{\mathcal{Z}}^{\bar{Q} \bar{R}} \Omega_{\bar{R} K}(\mathcal{Z} \Omega)^{K}+\text { h.c. }\right]+\mathcal{O}\left(\Omega^{5}\right)
\end{aligned}
$$

where the functions on the right-hand side depend on the fields $\mathcal{Y}^{I}$ and $\overline{\mathcal{Y}}^{I}$. We used the obvious notation $(\mathcal{Z} \Omega)^{I}=\mathcal{Z}^{I J} \Omega_{J}$ and $(\overline{\mathcal{Z}} \bar{\Omega})^{I}=\overline{\mathcal{Z}}^{\bar{I} \bar{J}} \bar{\Omega}_{\bar{J}}$. It is remarkable that the matrix $N^{I J}$ no longer appears in this relation. This is due to a subtle interplay of the various contributions to this result, which involves the ones coming from (3.15). On closer inspection, the result (3.19) turns out to be identical (modulo an overall factor 4) to $\mathcal{H}^{(1)}$ given in (3.18) upon making the replacement $N^{I J} \rightarrow \mathrm{i} \mathcal{Z}^{I J}$ and/or $N^{I J} \rightarrow-\mathrm{i} \overline{\mathcal{Z}}^{\bar{I} \bar{J}}$, where the precise form depends on the type of index contractions (i.e holomorphic or antiholomorphic) to $N^{I J}$. The fact that none of the other functions $\mathcal{H}^{(a)}$ contribute is perhaps not surprising because those are manifestly symplectic functions where no $\mathcal{Z}$ dependent variations are generated, whereas in $\mathcal{H}^{(1)}$ such terms are generated and have to be absorbed into the transformation rule of $\Omega$. Clearly this is an intriguing result for which we have not found a general proof within our approach, although we are aware of the fact that similar properties have been encountered in [26].

With the above result (3.19) one can continue with the iterations by extending (3.15) to the next order, finding

$$
\tilde{\Omega}_{I}(\tilde{\mathcal{Y}}, \overline{\mathcal{Y}})=\left[\mathcal{S}^{-1}\right]_{I}^{J}\left[\Omega_{J}+\mathrm{i} F_{J K L}(\mathcal{Z} \Omega)^{K}(\mathcal{Z} \Omega)^{L}-2 \mathrm{i} \Omega_{J K}(\mathcal{Z} \Omega)^{K}+2 \mathrm{i} \Omega_{J \bar{K}}(\overline{\mathcal{Z}} \bar{\Omega})^{\bar{K}}\right.
$$




$$
\begin{aligned}
& +\frac{2}{3} F_{J K L P}(\mathcal{Z} \Omega)^{K}(\mathcal{Z} \Omega)^{L}(\mathcal{Z} \Omega)^{P}+2 F_{K L P}(\mathcal{Z} \Omega)^{K}{ }_{J}(\mathcal{Z} \Omega)^{L}(\mathcal{Z} \Omega)^{P} \\
& +4 F_{J K L}(\mathcal{Z} \Omega)^{K}(\mathcal{Z} \Omega)^{L}{ }_{P}(\mathcal{Z} \Omega)^{P}-4 F_{J K L}(\mathcal{Z} \Omega)^{K}(\mathcal{Z} \Omega)^{L}{ }_{\bar{P}}(\overline{\mathcal{Z}} \bar{\Omega})^{\bar{P}} \\
& -2 F_{J K L} \mathcal{Z}^{L P} F_{P Q S}(\mathcal{Z} \Omega)^{K}(\mathcal{Z} \Omega)^{Q}(\mathcal{Z} \Omega)^{S}+2 \bar{F}_{\bar{K} \bar{L} \bar{P}}(\overline{\mathcal{Z}} \bar{\Omega})^{\bar{K}}{ }_{J}(\overline{\mathcal{Z}} \bar{\Omega})^{\bar{L}}(\overline{\mathcal{Z}} \bar{\Omega})^{\bar{P}} \\
& -2 \Omega_{J K L}(\mathcal{Z} \Omega)^{K}(\mathcal{Z} \Omega)^{L}-4 \Omega_{K L}(\mathcal{Z} \Omega)^{K}{ }_{J}(\mathcal{Z} \Omega)^{L}-2 \Omega_{J \bar{K} \bar{L}}(\overline{\mathcal{Z}} \bar{\Omega})^{\bar{K}}(\overline{\mathcal{Z}} \bar{\Omega})^{\bar{L}} \\
& -4 \Omega_{\bar{K} \bar{L}}(\overline{\mathcal{Z}} \bar{\Omega})^{\bar{K}}{ }_{J}(\overline{\mathcal{Z}} \bar{\Omega})^{\bar{L}}+4 \Omega_{J K \bar{L}}(\mathcal{Z} \Omega)^{K}(\overline{\mathcal{Z}} \bar{\Omega})^{\bar{L}}+4 \Omega_{K \bar{L}}(\mathcal{Z} \Omega)^{K}{ }_{J}(\overline{\mathcal{Z}} \bar{\Omega})^{\bar{L}} \\
& \left.+4 \Omega_{K \bar{L}}(\mathcal{Z} \Omega)^{K}(\overline{\mathcal{Z}} \bar{\Omega})^{\bar{L}}{ }_{J}\right]+\mathcal{O}\left(\Omega^{4}\right), \\
& \tilde{\Omega}_{I J}=\left[\mathcal{S}^{-1}\right]^{K}{ }_{I}\left[\mathcal{S}^{-1}\right]^{L}{ }_{J}\left[\Omega_{K L}-F_{K L M} \mathcal{Z}^{M N} \Omega_{N}+\mathrm{i} F_{K L M N}(\mathcal{Z} \Omega)^{M}(\mathcal{Z} \Omega)^{N}\right. \\
& +2 \mathrm{i} F_{K M N}(\mathcal{Z} \Omega)^{M}{ }_{L}(\mathcal{Z} \Omega)^{N}-2 \mathrm{i} F_{K M N} \mathcal{Z}^{M P} F_{P Q L}(\mathcal{Z} \Omega)^{Q}(\mathcal{Z} \Omega)^{N} \\
& -2 \mathrm{i} \Omega_{K L P}(\mathcal{Z} \Omega)^{P}-2 \mathrm{i} \Omega_{K P}(\mathcal{Z} \Omega)^{P}{ }_{L}+2 \mathrm{i} \Omega_{K P} \mathcal{Z}^{P Q} F_{Q L S}(\mathcal{Z} \Omega)^{S} \\
& \left.+2 \mathrm{i} \Omega_{K L \bar{P}}(\overline{\mathcal{Z}} \bar{\Omega})^{\bar{P}}+2 \mathrm{i} \Omega_{K \bar{P}}(\overline{\mathcal{Z}} \bar{\Omega})^{\bar{P}}{ }_{L}\right]+\mathcal{O}\left(\Omega^{3}\right), \\
& \tilde{\Omega}_{I \bar{J}}=\left[\mathcal{S}^{-1}\right]^{K}{ }_{I}\left[\overline{\mathcal{S}}^{-1}\right]^{\bar{L}}{ }_{\bar{J}}\left[\Omega_{K \bar{L}}+2 \mathrm{i} F_{K M N}(\mathcal{Z} \Omega)^{M}{ }_{\bar{L}}(\mathcal{Z} \Omega)^{N}-2 \mathrm{i} \bar{F}_{\bar{L} \bar{P} \bar{N}}(\overline{\mathcal{Z}} \bar{\Omega})^{\bar{N}}{ }_{K}(\overline{\mathcal{Z}} \bar{\Omega})^{\bar{P}}\right. \\
& \left.-2 \mathrm{i} \Omega_{K M \bar{L}}(\mathcal{Z} \Omega)^{M}-2 \mathrm{i} \Omega_{K M}(\mathcal{Z} \Omega)^{M}{ }_{\bar{L}}+2 \mathrm{i} \Omega_{K \bar{L} \bar{M}}(\overline{\mathcal{Z}} \bar{\Omega})^{\bar{M}}+2 \mathrm{i} \Omega_{K \bar{M}}(\overline{\mathcal{Z}} \bar{\Omega})^{\bar{M}}{ }_{\bar{L}}\right] \\
& +\mathcal{O}\left(\Omega^{3}\right) \text {, }
\end{aligned}
$$

where $(\mathcal{Z} \Omega)^{M}{ }_{L}=\mathcal{Z}^{M N} \Omega_{N L},(\overline{\mathcal{Z}} \bar{\Omega})^{\bar{P}}{ }_{L}=\overline{\mathcal{Z}}^{\bar{P} \bar{N}} \Omega_{\bar{N} L},(\mathcal{Z} \Omega)^{L}{ }_{\bar{P}}=\mathcal{Z}^{L K} \Omega_{K \bar{P}}$, etc. These results will then contribute to the determination of the next order contribution to (3.19). The fact that $\Omega$ transforms non-linearly under dualities, while we are at the same time considering an expansion in terms of $\Omega$ and its derivatives, suggests to introduce a formal expansion parameter $\alpha$ and expand $\Omega=\sum_{n=1}^{\infty} \alpha^{n-1} \Omega^{(n)}$, so that we are obtaining relations between products of different coefficient functions $\Omega^{(n)}$ order-in-order in $\alpha$. At this stage there is no direct need for this, but we will follow this strategy in the next section where matters become somewhat more involved.

Rather than proceeding with this iteration procedure, we will simply assume that all characteristic features noted in the results above, will continue to hold in higher orders as well. An obvious conclusion is then that the quantity $\Omega$ does not transform as a function under symplectic transformations in view of the result (3.19). This is in agreement with our earlier claims, for instance in $[15,16]$. On the other hand we expect that $\Omega$ must belong to a restricted class and, in particular, it should have a well-defined harmonic limit that will define the Wilsonian action. To understand how this may come about, let us first start from an $\Omega$ that is harmonic in the variables $\mathcal{Y}^{I}$ and their complex conjugates $\overline{\mathcal{Y}}^{I}$. It is then reasonable to expect that also $\tilde{\Omega}$ will be harmonic in the new variables, so that both $\Omega$ and $\tilde{\Omega}$ can be written as a sum of a holomorphic and an anti-holomorphic function in their respective variables. Indeed this property is confirmed by (3.19), or alternatively by the last equation in (3.20). Hence we conclude that the condition

$$
\Omega_{I \bar{J}}=0,
$$

is preserved under symplectic transformations. In the next section we first discuss the consequences of this harmonicity constraint, before considering modifications thereof. 


\section{Non-holomorphic deformations and the anomaly equation}

At the end of the previous section we mentioned the possibility that $\Omega$ is an harmonic function of the variables $\mathcal{Y}^{I}$ and $\overline{\mathcal{Y}}^{I}$, a property that is consistent with respect to symplectic transformations. However, the function of interest is $\mathcal{H}^{(1)}(\mathcal{Y}, \overline{\mathcal{Y}})$, which is not harmonic in this case but which can still be decomposed in a way that is rather similar to the decomposition of a harmonic function. Namely one can write,

$$
\begin{aligned}
\Omega(\mathcal{Y}, \overline{\mathcal{Y}}) & =\omega(\mathcal{Y})+\bar{\omega}(\overline{\mathcal{Y}}), \\
\mathcal{H}^{(1)}(\mathcal{Y}, \overline{\mathcal{Y}}) & =h(\mathcal{Y}, \overline{\mathcal{Y}})+\bar{h}(\overline{\mathcal{Y}}, \mathcal{Y}),
\end{aligned}
$$

where the function $h(\mathcal{Y}, \overline{\mathcal{Y}})$ depends holomorphically on $\omega$ and equals,

$$
\begin{aligned}
h(\mathcal{Y}, \overline{\mathcal{Y}})= & 4 \omega-4 N^{I J} \omega_{I} \omega_{J} \\
& +8 \omega_{I J}(N \omega)^{I}(N \omega)^{J}+\frac{8}{3} \mathrm{i} F_{I J K}(N \omega)^{I}(N \omega)^{J}(N \omega)^{K} \\
& -\frac{4}{3} \mathrm{i}\left(F_{I J K L}+3 \mathrm{i} F_{R(I J} N^{R S} F_{K L) S}\right)(N \omega)^{I}(N \omega)^{J}(N \omega)^{K}(N \omega)^{L} \\
& -\frac{16}{3} \omega_{I J K}(N \omega)^{I}(N \omega)^{J}(N \omega)^{K}-16 \mathrm{i} F_{I J K} N^{K P} \omega_{P Q}(N \omega)^{I}(N \omega)^{J}(N \omega)^{Q} \\
& -16(N \omega)^{P} \omega_{P Q} N^{Q R} \omega_{R K}(N \omega)^{K}+\mathcal{O}\left(\omega^{5}\right) .
\end{aligned}
$$

Because both $\mathcal{H}^{(1)}$ and $\tilde{\Omega}$, given in (3.18) and (3.19), are now harmonic in the $\omega$, it follows that $\omega$ must transform under symplectic transformations in direct correspondence with (3.19),

$$
\begin{aligned}
\tilde{\omega}(\tilde{\mathcal{Y}})= & \omega-\mathrm{i} \mathcal{Z}^{I J} \omega_{I} \omega_{J}+\frac{2}{3} F_{I J K}(\mathcal{Z} \omega)^{I}(\mathcal{Z} \omega)^{J}(\mathcal{Z} \omega)^{K} \\
& -2 \omega_{I J}(\mathcal{Z} \omega)^{I}(\mathcal{Z} \omega)^{J} \\
& -\frac{1}{3} \mathrm{i} F_{I J K L}(\mathcal{Z} \omega)^{I}(\mathcal{Z} \omega)^{J}(\mathcal{Z} \omega)^{K}(\mathcal{Z} \omega)^{L} \\
& +\frac{4}{3} \mathrm{i} \omega_{I J K}(\mathcal{Z} \omega)^{I}(\mathcal{Z} \omega)^{J}(\mathcal{Z} \omega)^{K} \\
& +\mathrm{i} F_{I J K} \mathcal{Z}^{K L} F_{L M N}(\mathcal{Z} \omega)^{I}(\mathcal{Z} \omega)^{J}(\mathcal{Z} \omega)^{M}(\mathcal{Z} \omega)^{N} \\
& -4 \mathrm{i} F_{I J K} \mathcal{Z}^{K P} \omega_{P Q}(\mathcal{Z} \omega)^{I}(\mathcal{Z} \omega)^{J}(\mathcal{Z} \omega)^{Q} \\
& +4 \mathrm{i}(\mathcal{Z} \omega)^{I} \omega_{I J} \mathcal{Z}^{J K} \omega_{K L}(\mathcal{Z} \omega)^{L}+\mathcal{O}\left(\omega^{5}\right),
\end{aligned}
$$

so that $\omega$ transforms holomorphically. Obviously $h(\mathcal{Y}, \overline{\mathcal{Y}})$ must be a symplectic function. However, its dependence on $\overline{\mathcal{Y}}$ resides exclusively in the complex matrix $N^{I J}$. Therefore we first study the dependence of $h(\mathcal{Y}, \overline{\mathcal{Y}})$ on $N^{I J}$, and derive the following equation

$$
\frac{\partial h}{\partial N^{I J}}=-\frac{1}{4} \partial_{I} h \partial_{J} h
$$

which we have verified up to terms of order $\omega^{5}$. This result can easily be understood on the basis of the diagrammatic interpretation that we have presented in the previous section. From it one straightforwardly determines the non-holomorphic derivative of $h(\mathcal{Y}, \overline{\mathcal{Y}})$,

$$
\partial_{\bar{I}} h(\mathcal{Y}, \overline{\mathcal{Y}})=\frac{1}{4} \mathrm{i} \bar{F}_{I K L} N^{K M} N^{L N} \partial_{M} h(\mathcal{Y}, \overline{\mathcal{Y}}) \partial_{N} h(\mathcal{Y}, \overline{\mathcal{Y}})
$$


The equation (4.5) partially coincides with what is known as the holomorphic anomaly equation for the topological string and represents the terms that are induced by the pinching of a cycle of the underlying Riemann surface resulting in two disconnected Riemann surfaces [11]. This restricted anomaly equation is also obtained when considering the $N=2$ chiral superspace action for abelian vector multiplets in the presence of a chiral background field [34]. When expanding this action in terms of the background, the holomorphic expansion coefficient functions do not transform as functions under duality. This can be resolved by covariantizing the Taylor expansion with a suitable connection, but in that case the expansion coefficient functions are no longer holomorphic. As it turns out these modified coefficient functions then satisfy the same anomaly equation (4.5). Note that this equation is integrable, so that no additional constraints are implied. Clearly the holomorphic anomaly is due to the fact that there is a conflict between symplectic covariance and holomorphicity.

The above result would justify the identification of $\mathcal{H}^{(1)}$ with the topological string, except that the holomorphic anomaly equation is still incomplete. This implies that we have to somehow relax the assumption that $\Omega$ is harmonic in $\mathcal{Y}$, while still expressing it in terms of a holomorphic function $\omega(\mathcal{Y})$ in such a way that $\mathcal{H}^{(1)}$ will remain harmonic (possibly up to a separate non-harmonic function, as we shall see in section 5) in terms of $\omega$. However, modifying the ansatz (4.1) must be consistent with duality, in the sense that the modification will hold for the whole class of functions $\Omega$ that are related by duality. Our task is therefore to demonstrate that the present framework can be extended so as to induce the remaining term in the anomaly equation that is related to pinchings of cycles of the Riemann surface that reduce the genus by one unit.

As it turns out, a consistent extension can be constructed by introducing non-harmonic terms whose variation under symplectic transformations is still harmonic. Such a modification does preserve the present framework in a way that is consistent with duality. For instance we could choose the following ansatz for $\Omega$,

$$
\Omega(\mathcal{Y}, \overline{\mathcal{Y}})=\omega(\mathcal{Y})+\bar{\omega}(\overline{\mathcal{Y}})+\alpha \ln \operatorname{det}\left[N_{I J}\right]+\beta \Psi(\mathcal{Y}, \overline{\mathcal{Y}})
$$

where $\alpha$ and $\beta$ are arbitrary real parameters and $\Psi$ a non-holomorphic function of $\mathcal{Y}$ and $\overline{\mathcal{Y}}$. Note that we assume that the two deformations do not depend on the holomorphic function $\omega$ or its complex conjugate, because we insist on harmonicity with respect to $\omega$.

The deformation proportional to $\beta$ is the easiest to deal with, so let us consider this one first. Since $\Psi$ is a given function one cannot simply substitute the ansatz (4.6) with $\alpha=0$ into the expression for the function $\mathcal{H}^{(1)}$, because this would require $\Psi$ to change non-trivially in order to satisfy (3.19). Hence we must introduce additional terms into $\Omega$ to ensure that the modified $\Omega$ will still transform according to (3.19) without modifying the holomorphicity of $\omega$ and leaving $\Psi$ as a function. As its turns out, this can be done in the next order by including the following additional terms,

$$
\Omega(\mathcal{Y}, \overline{\mathcal{Y}})=\omega+\bar{\omega}+\beta \Psi(\mathcal{Y}, \overline{\mathcal{Y}})+N^{I J}\left[2 \beta \partial_{I} \omega \partial_{J} \Psi+\beta^{2} \partial_{I} \Psi \partial_{J} \Psi+\text { h.c. }\right] .
$$

So far we have been performing an interation in powers of $\Omega$. It is formally consistent to treat $\beta$ and $\omega$ as being of the same order, but then we must assume that $\omega$ can obtain 
terms of even higher order in $\beta$ in view of the non-linear transformation rules, Hence we assume $\omega$ can be expanded in terms of the parameter $\beta$,

$$
\omega(\mathcal{Y}) \longrightarrow \omega(\mathcal{Y}, \beta)=\sum_{n=1}^{\infty} \omega_{n}(\mathcal{Y}) \beta^{n-1}
$$

although we will keep this expansion implicit in what follows. It is now straightforward to see that $\omega$ transforms as follows under symplectic transformations

$$
\tilde{\omega}=\omega-\mathrm{i} \mathcal{Z}^{I J} \omega_{I} \omega_{J}+\mathcal{O}\left(\omega^{3}\right),
$$

which agrees with (4.3). Subsequently we consider the function $\mathcal{H}^{(1)}$ in this order of iteration,

$$
\mathcal{H}^{(1)}=h+\bar{h}+4 \beta \Psi+\mathcal{O}\left(\omega^{3}\right),
$$

where $h$ coincides with (4.2) in this order of iteration. Consequently the addition of such a deformation leaves the holomorphic anomaly equation (4.5) unaltered.

In view of this result we continue with the first modification in (4.6) proportional to $\alpha$. In principle the analysis proceeds in a similar way as in the previous case, but here one has to also investigate the consistency in first order. However, it is easy to see how consistency can be achieved because we have

$$
\ln \operatorname{det}\left[\tilde{N}_{I J}\right]=\ln \operatorname{det}\left[N_{I J}\right]-\ln \operatorname{det}[\mathcal{S}]-\ln \operatorname{det}[\overline{\mathcal{S}}],
$$

where $\mathcal{S}$ was defined in (2.9). Because $\mathcal{S}$ is holomorphic, the effect of the non-harmonic modification $\alpha \ln \operatorname{det}\left[N_{I J}\right]$ under duality can simply be absorbed by assigning the following transformation to $\omega$,

$$
\tilde{\omega}(\tilde{\mathcal{Y}})=\omega(\mathcal{Y})+\alpha \ln \operatorname{det}[\mathcal{S}]
$$

up to terms of higher order in $\Omega$ (or $\omega$ ) and $\alpha$. Hence in lowest order $\Omega(\mathcal{Y}, \overline{\mathcal{Y}}$ ) transforms as a function, so that our previous analysis remains unaffected as $\tilde{\Omega}=\Omega+\mathcal{O}\left(\Omega^{2}\right)$. This is confirmed by the following. First of all, derivatives of the holomorphic function $\omega(\mathcal{Y})$ remain holomorphic but they do acquire extra terms in their transformation rules, as is shown in

$$
\begin{aligned}
& \tilde{\omega}_{I}=\left[\mathcal{S}^{-1}\right]_{I}^{J}\left(\omega_{J}+\alpha F_{J K L} \mathcal{Z}^{K L}\right)+\mathcal{O}\left(\alpha^{2}\right) \\
& \begin{aligned}
\tilde{\omega}_{I J}=\left[\mathcal{S}^{-1}\right]_{I}^{K}\left[\mathcal{S}^{-1}\right]_{J}^{L}\left[\omega_{K L}-F_{K L M} \mathcal{Z}^{M N}\left(\omega_{N}+\alpha F_{N P Q} \mathcal{Z}^{P Q}\right)\right. \\
\\
\left.\quad+\alpha\left(F_{K L M N}-F_{K M P} F_{L N Q} \mathcal{Z}^{P Q}\right) \mathcal{Z}^{M N}\right]+\mathcal{O}\left(\alpha^{2}\right) .
\end{aligned}
\end{aligned}
$$

Furthermore the first few derivatives of $\Omega$ are now equal to

$$
\begin{aligned}
\Omega_{I} & =\omega_{I}-\mathrm{i} \alpha F_{I J K} N^{J K}+\mathcal{O}\left(\alpha^{2}\right), \\
\Omega_{I J} & =\omega_{I J}+\alpha F_{I K L} F_{J M N} N^{K M} N^{L N}-\mathrm{i} \alpha F_{I J K L} N^{K L}+\mathcal{O}\left(\alpha^{2}\right), \\
\Omega_{I \bar{J}} & =-\alpha F_{I K L} \bar{F}_{J M N} N^{K M} N^{L N}+\mathcal{O}\left(\alpha^{2}\right),
\end{aligned}
$$


which, in leading order, transform consistently under duality transformations (i.e. according to (3.20)). To show this one makes use of (4.13) and (2.8). Hence we conclude that to lowest order in $\Omega$, the non-holomorphic deformation (4.6) is consistent. Observe that the last equation (4.14) constitutes a deviation from the harmonicity condition (3.21).

In due course we will also need the result for the transformation of the third- and fourth-derivatives of $\omega$, implied by (4.12),

$$
\begin{aligned}
& \left.\tilde{\omega}_{I J K}=\left[\mathcal{S}^{-1}\right]^{M}{ }_{(I}\left[\mathcal{S}^{-1}\right]^{N}{ }_{J}\left[\mathcal{S}^{-1}\right]^{P}{ }_{K}\right) \\
& \times\left[\omega_{M N P}-3 F_{M N \bullet} \mathcal{Z}^{\bullet \bullet}\left(\omega_{P \bullet}+\alpha F_{P Q R \bullet} \mathcal{Z}^{Q R}\right)\right. \\
& -\left(F_{M N P \bullet}-3 F_{M N \bullet} \mathcal{Z}^{\bullet \bullet} F_{P \bullet \bullet}\right) \mathcal{Z}^{\bullet \bullet}\left(\omega_{\bullet}+\alpha F_{Q R \bullet} \mathcal{Z}^{Q R}\right) \\
& +\alpha\left(F_{M N P Q R}-3 F_{M N Q \bullet} \mathcal{Z}^{\bullet \bullet} F_{P R \bullet}+3 F_{M N} \bullet \mathcal{Z}^{\bullet \bullet} F_{Q \bullet \bullet} \mathcal{Z}^{\bullet \bullet} F_{\bullet P R}\right) \mathcal{Z}^{Q R} \\
& \left.+2 \alpha F_{M Q \bullet} \mathcal{Z}^{\bullet \bullet} F_{\bullet \bullet N} \mathcal{Z}^{\bullet \bullet} F_{P R \bullet} \mathcal{Z}^{Q R}\right]+\mathcal{O}\left(\alpha^{2}\right), \\
& \tilde{\omega}_{I J K L}=\left[\mathcal{S}^{-1}\right]_{(I}^{M}\left[\mathcal{S}^{-1}\right]^{N}{ }_{J}\left[\mathcal{S}^{-1}\right]^{P}{ }_{K}\left[\mathcal{S}^{-1}\right]^{Q}{ }_{L)} \\
& \times\left[\omega_{M N P Q}-6 F_{M N \bullet} \mathcal{Z}^{\bullet \bullet}\left(\omega_{P Q}+\alpha F_{P Q U V} \bullet \mathcal{Z}^{U V}\right)\right. \\
& -4\left(F_{M N P \bullet}-3 F_{M N \bullet} \mathcal{Z}^{\bullet \bullet} F_{P \bullet \bullet}\right) \mathcal{Z}^{\bullet \bullet}\left(\omega_{Q \bullet}+\alpha F_{Q \bullet U V} \mathcal{Z}^{U V}\right) \\
& +3 F_{M N \bullet} \mathcal{Z}^{\bullet \bullet}\left(\omega_{\bullet \bullet}+\alpha F_{\bullet \bullet U V} \mathcal{Z}^{U V}\right) \mathcal{Z}^{\bullet \bullet} F_{\bullet P Q} \\
& -\left(F_{M N P Q \bullet}-4 F_{M N P \bullet} \mathcal{Z}^{\bullet \bullet} F_{Q \bullet \bullet}-6 F_{M N \bullet} \mathcal{Z}^{\bullet \bullet} F_{P Q \bullet \bullet}\right) \mathcal{Z}^{\bullet \bullet}\left(\omega_{\bullet}+\alpha F_{U V \bullet} \mathcal{Z}^{U V}\right) \\
& -12 F_{M N \bullet} \mathcal{Z}^{\bullet \bullet} F_{P \bullet \bullet} \mathcal{Z}^{\bullet \bullet} F_{Q \bullet \bullet} \mathcal{Z}^{\bullet \bullet}\left(\omega_{\bullet}+\alpha F_{U V \bullet} \mathcal{Z}^{U V}\right) \\
& -3\left(\mathcal{Z}^{R \bullet} F_{M N \bullet}\right)\left(\mathcal{Z}^{S \bullet} F_{P Q \bullet}\right) F_{R S T} \mathcal{Z}^{T \bullet}\left(\omega_{\bullet}+\alpha F_{U V \bullet} \mathcal{Z}^{U V}\right) \\
& +\alpha\left[F_{M N P Q U V} \mathcal{Z}^{U V}-4 F_{M N P U V} \mathcal{Z}^{U \bullet} F_{Q \bullet \bullet} \mathcal{Z}^{\bullet V}-3 F_{M N X U} F_{P Q Y V} \mathcal{Z}^{X Y} \mathcal{Z}^{U V}\right] \\
& +4 \alpha\left[3\left(F_{M N \bullet \bullet} \mathcal{Z}^{\bullet \bullet} F_{P \bullet \bullet} \mathcal{Z}^{\bullet \bullet} F_{Q \bullet \bullet} \mathcal{Z}^{\bullet \bullet}\right)+F_{M N P \bullet} \mathcal{Z}^{\bullet X}\left(F_{X \bullet \bullet} \mathcal{Z}^{\bullet \bullet} F_{Q \bullet \bullet} \mathcal{Z}^{\bullet \bullet}\right)\right] \\
& +6 \alpha\left[2 F_{M N} \mathcal{Z}^{\bullet X}\left(F_{X P \bullet \bullet} \mathcal{Z}^{\bullet \bullet} F_{Q \bullet \bullet} \mathcal{Z}^{\bullet \bullet}\right)+F_{M N} \bullet \mathcal{Z}^{\bullet X}\left(F_{X \bullet \bullet} \mathcal{Z}^{\bullet \bullet} F_{P Q \bullet \bullet} \mathcal{Z}^{\bullet \bullet}\right)\right] \\
& -6 \alpha\left(F_{M \bullet \bullet} \mathcal{Z}^{\bullet \bullet} F_{N \bullet \bullet} \mathcal{Z}^{\bullet \bullet} F_{P \bullet \bullet} \mathcal{Z}^{\bullet \bullet} F_{Q \bullet \bullet} \mathcal{Z}^{\bullet \bullet}\right) \\
& -12 \alpha F_{M N \bullet} \mathcal{Z}^{\bullet \bullet} F_{P \bullet \bullet} \mathcal{Z}^{\bullet X}\left(F_{X \bullet \bullet} \mathcal{Z}^{\bullet \bullet} F_{Q \bullet \bullet} \mathcal{Z}^{\bullet \bullet}\right) \\
& -12 \alpha F_{M N \bullet} \mathcal{Z}^{\bullet X}\left(F_{X \bullet \bullet} \mathcal{Z}^{\bullet \bullet} F_{P \bullet \bullet} \mathcal{Z}^{\bullet \bullet} F_{Q \bullet \bullet} Z^{\bullet \bullet}\right) \\
& -3 \alpha F_{M N \bullet} \mathcal{Z}^{\bullet X}\left(F_{X \bullet \bullet} \mathcal{Z}^{\bullet \bullet} F_{Y \bullet \bullet} \mathcal{Z}^{\bullet \bullet}\right) \mathcal{Z}^{Y \bullet} F_{\bullet P Q}+\mathcal{O}\left(\alpha^{2}\right) \text {. }
\end{aligned}
$$

To continue this scheme to higher orders in $\Omega$ is not an easy task. So far we have been working order-by-order in powers of $\Omega$, but now we are dealing also with additional terms that are proportional to the parameter $\alpha$. Within the iterative procedure that we have been following it is consistent to formally treat $\omega$ and $\alpha$ as being of the same order as $\Omega$. Counting in this way shows that the corrections in (4.15) are of first order in $\alpha$. Because the equations that we are dealing with are non-linear it is therefore imperative that the $\omega$ itself can in principle contain contributions of arbitrary order in $\alpha$, a possibility that we have already been alluding to below equation (3.20). Therefore we will in addition assume that $\omega$ can be expanded in terms of $\alpha$,

$$
\omega(\mathcal{Y}) \longrightarrow \omega(\mathcal{Y}, \alpha)=\sum_{n=1}^{\infty} \omega_{n}(\mathcal{Y}) \alpha^{n-1},
$$

although we will keep this expansion implicit in what follows. Assuming that $\omega$ incorporates the higher-order terms in $\alpha$ we can proceed to higher orders by iteration to obtain the 
extension of the original almost-harmonic ansatz (4.6), possibly up to terms that separately constitute proper functions under symplectic transformations.

We thus continue to derive the terms of order $\alpha^{2}$, which can be found from (3.19),

$$
\begin{aligned}
\tilde{\Omega}-\Omega= & -\mathrm{i}\left(\mathcal{Z}^{I J} \Omega_{I} \Omega_{J}-\overline{\mathcal{Z}}^{I J} \Omega_{\bar{I}} \Omega_{\bar{J}}\right)+\mathcal{O}\left(\Omega^{3}\right) \\
= & -\mathrm{i} \mathcal{Z}^{I J}\left[\omega_{I} \omega_{J}-2 \mathrm{i} \alpha \omega_{I} F_{J K L} N^{K L}-\alpha^{2} F_{I K L} F_{J M N} N^{K L} N^{M N}\right]+\text { h.c. } \\
& +\mathcal{O}\left(\alpha^{3}\right),
\end{aligned}
$$

where we note that the right-hand side is of order $\alpha^{2}$, as both $\alpha$ and $\omega$ are counted as being of the same order as $\Omega$. Our task is now to include further modifications into $\Omega$, just as we did earlier in (4.6), so that the change of $\Omega$ under a symplectic transformation becomes consistent with the right-hand side of (4.17), up to a term that is harmonic, which can then be absorbed into the variation of $\omega$. The problem here is, however, that the expression (4.17) is linear in $\mathcal{Z}^{I J}$ but involves also terms that are linear or quadratic in $N^{I J}$. It is clear that one cannot construct a suitable addition to $\Omega$ depending exclusively on $\omega_{I}, N^{I J}$ and $F_{I J K}$. There exists, however, an alternative, namely to include higher derivatives of $\omega$. According to (4.13) the second derivative $\omega_{I J}$ leads to similar variations, suggesting another possible modification. Indeed, it follows that $N^{I J} \omega_{I J}, F_{I J K L} N^{I J} N^{K L}$ and $F_{I J K} F_{L M N} N^{I L} N^{J M} N^{K N}$ (and complex conjugates where appropriate) are the terms that one may add to $\Omega$ so that only a holomorphic variation will remain in (4.17). And indeed, one can verify by explicit calculation that $\Omega$ should be written as (up to a symplectic function of $\mathcal{Y}$ and $\overline{\mathcal{Y}}$ ),

$$
\begin{aligned}
\Omega(\mathcal{Y}, \overline{\mathcal{Y}})= & \alpha \ln N \\
& +\left[\omega+2 \alpha N^{I J} \omega_{I J}-\alpha^{2}\left[\mathrm{i} F_{I J K L}-\frac{2}{3} F_{I K M} F_{J L N} N^{M N}\right] N^{I J} N^{K L}+\text { h.c. }\right] \\
& +\mathcal{O}\left(\alpha^{3}\right),
\end{aligned}
$$

where here and henceforth we use the definition $N \equiv \operatorname{det}\left[N_{I J}\right]$. Hence the holomorphic function $\omega$ is now accompanied by a variety of specific non-holomorphic modifications which will contribute to the effective action. Indeed, with this result for $\Omega(\mathcal{Y}, \overline{\mathcal{Y}})$, explicit evaluation shows that (4.17) is satisfied up to order $\alpha^{3}$ provided that $\omega$ transforms (holomorphically) according to

$$
\begin{aligned}
\tilde{\omega}= & \omega+\alpha \ln \operatorname{det}[\mathcal{S}]+\mathrm{i} \mathcal{Z}^{I J}\left[2 \alpha \omega_{I J}-\left(\omega_{I}+\alpha F_{I K L} \mathcal{Z}^{K L}\right)\left(\omega_{J}+\alpha F_{J M N} \mathcal{Z}^{M N}\right)\right] \\
& +\mathrm{i} \alpha^{2}\left[F_{I J K L}-\frac{2}{3} F_{I K M} F_{J L N} \mathcal{Z}^{M N}\right] \mathcal{Z}^{I J} \mathcal{Z}^{K L}+\mathcal{O}\left(\alpha^{3}\right) .
\end{aligned}
$$

To see this one makes use of the transformations of multiple derivatives of the holomorphic function $F(\mathcal{Y})$, listed in (2.8), the transformation of $N^{I J}$ as given in (2.13), and the transformation rule for $\omega_{I J}$ specified in the second equation of (4.13). Incidentally, the result (4.18) is in line with the ansatz (4.16) according to which the function $\omega$ is expanded in powers of $\alpha$. Furthermore it turns out that the result (4.18) takes the form of a sum over connected 1PI diagrams, unlike the corresponding result for $\mathcal{H}^{(1)}$. 
Let us now return to the almost harmonic function $\mathcal{H}^{(1)}(\mathcal{Y}, \overline{\mathcal{Y}} ; N)$, which now decomposes according to

$$
\mathcal{H}^{(1)}(\mathcal{Y}, \overline{\mathcal{Y}} ; N)=4 \alpha \ln N+h(\mathcal{Y}, \overline{\mathcal{Y}})+\bar{h}(\overline{\mathcal{Y}}, \mathcal{Y})
$$

which turns out to be a harmonic function of $\omega$. Indeed, making use of (4.18) one finds that $h(\mathcal{Y}, \overline{\mathcal{Y}})$ now takes the form,

$$
\begin{aligned}
h(\mathcal{Y}, \overline{\mathcal{Y}})= & 4 \omega-4\left(\omega_{I}-\mathrm{i} \alpha F_{I K L} N^{K L}\right) N^{I J}\left(\omega_{J}-\mathrm{i} \alpha F_{J M N} N^{M N}\right) \\
& +8 \alpha \omega_{I J} N^{I J}-4 \alpha^{2}\left[\mathrm{i} F_{I J K L}-\frac{2}{3} F_{I K M} F_{J L N} N^{M N}\right] N^{I J} N^{K L}+\mathcal{O}\left(\alpha^{3}\right) .
\end{aligned}
$$

We stress that, by construction, $\mathcal{H}^{(1)}$ remains a symplectic function in the presence of the term $4 \alpha \ln N$ in (4.20). Furthermore $h$ will separately transform as a function beyond linear order in $\alpha$ and derivatives of $h+4 \alpha \ln N$ will still transform as proper tensors. This must be the case because the transformations of $h$ beyond the linear order depend only on $\mathcal{S}$ through the tensor $\mathcal{Z}^{I J}$, and likewise $\bar{h}$ depends only on $\overline{\mathcal{S}}$ through the tensor $\overline{\mathcal{Z}}^{I J}$. As we shall see, the non-holomorphic derivative of $h$ will transform as a vector as it is not of first order in $\alpha$. We also observe that the transformation of $\omega$ as specified in (4.19) follows precisely from the expression for $\frac{1}{4} h(\mathcal{Y}, \overline{\mathcal{Y}})$ upon replacing $N^{I J}$ by $\mathrm{i} \mathcal{Z}^{I J}$, with the exception of the term $\alpha \ln \operatorname{det}[\mathcal{S}]$ that is related to the explicitly non-harmonic term in (4.18). This is in line with the phenomenon noted below equation (3.19).

Now we return to the holomorphic anomaly equation. Following the discussion at the beginning of this section we first determine,

$$
\begin{aligned}
\frac{\partial h}{\partial N^{I J}}= & -\frac{1}{4} \partial_{I}(h+4 \alpha \ln N) \partial_{J}(h+4 \alpha \ln N) \\
& +2 \alpha D_{I} \partial_{J}(h+4 \alpha \ln N)+\mathcal{O}\left(\alpha^{3}\right) .
\end{aligned}
$$

Here we have introduced a covariant derivative which ensures covariance under the symplectic transformations. On a holomorphic vector, $V_{I}$, this covariant derivative takes the form,

$$
D_{I} V_{J}=\partial_{I} V_{J}-\Gamma_{I J}{ }^{K} V_{K}
$$

where $\Gamma_{I J}{ }^{K}$ is Christoffel connection associated with the Kähler metric $g_{I \bar{J}}=\partial_{I} \partial_{\bar{J}} K(\mathcal{Y}, \overline{\mathcal{Y}})$ $=N_{I J}$, with $K$ the Kähler potential ${ }^{6}$

$$
K(\mathcal{Y}, \overline{\mathcal{Y}})=-\mathrm{i}\left[\overline{\mathcal{Y}}^{\bar{I}} F_{I}(\mathcal{Y})-\mathcal{Y}^{I} \bar{F}_{\bar{I}}(\overline{\mathcal{Y}})\right]
$$

Observe that, for a Kähler space the non-vanishing connection components are $\Gamma_{I J}{ }^{K}$ and its complex conjugate $\Gamma_{\bar{I} \bar{J}} \bar{K}$. The non-vanishing (up to complex conjugation) connection and curvature components are then equal to

$$
\begin{aligned}
\Gamma_{I J}{ }^{K} & =g^{K \bar{L}} \partial_{I} g_{J \bar{L}}=-\mathrm{i} F_{I J L} N^{L K}, \\
R_{\bar{I} J K}{ }^{L} & =\partial_{\bar{I}} \Gamma_{J K}{ }^{L}=-N^{L M} \bar{F}_{\bar{I} \bar{M} \bar{N}} N^{N P} F_{P J K} .
\end{aligned}
$$

\footnotetext{
${ }^{6}$ Note that there is no uniformity in the literature regarding the overall sign of $K$. See, e.g. [15].
} 
We remind the reader that combinations of higher derivatives of the holomorphic function $F(\mathcal{Y})$ that involve also the matrix $N^{I J}$ can transform covariantly under symplectic transformations, as was pointed out at the end of section 2 (see e.g. (2.14)).

We should also stress that the above discussion pertains to the underlying Kähler geometry. Obviously the special diffeomorphism related to the symplectic transformations form a subgroup of the group of holomorphic diffeomorphisms. This is confirmed by evaluating the transformation of the connection under a symplectic transformation, using the results presented in section 2 ,

$$
\Gamma_{I J}{ }^{K} \longrightarrow\left[\mathcal{S}^{-1}\right]_{I}^{L}\left[\mathcal{S}^{-1}\right]^{M}{ }_{J}\left[\Gamma_{L M}{ }^{N} \mathcal{S}^{K}{ }_{N}-\partial_{L} \mathcal{S}^{K}{ }_{M}\right]
$$

After these comments and clarifications we return to equation (4.22). Noting the lack of holomorphicity in $h$ resides in $N^{I J}$, we can now determine the anti-holomorphic derivative of $h$,

$$
\begin{aligned}
\partial_{\bar{I}} h= & \mathrm{i} \bar{F}_{I K L} N^{K M} N^{L N}\left[\frac{1}{4} \partial_{M}(h+4 \alpha \ln N) \partial_{N}(h+4 \alpha \ln N)-2 \alpha D_{M} \partial_{N}(h+4 \alpha \ln N)\right] \\
& +\mathcal{O}\left(\alpha^{3}\right)
\end{aligned}
$$

where the right-hand side contains no terms linear in $\alpha$. However, one could consider the mixed derivative of $h+4 \alpha \ln N$, which does contain terms linear in $\alpha$ given by

$$
\partial_{\bar{I}} \partial_{J}(h+4 \alpha \ln N)=-4 \alpha N^{K L} N^{M N} F_{J K M} \bar{F}_{I L N} .
$$

The expression on the right-hand side is precisely equal to $4 \alpha R_{\bar{I} J}$, where $R_{\bar{I} J}=R_{\bar{I} K J}{ }^{K}$ equals the Ricci tensor of the special Kähler manifold whose value follows from the second equation in (4.25). The equations (4.27) and (4.28) are the familiar holomorphic anomaly equations of the topological string.

In this section we introduced a deformation of $\Omega$ proportional to the parameter $\alpha$ which induced further corrections to $\Omega$ of higher orders in $\alpha$. This deformation was not itself a proper function, but its variation under a symplectic transformation was harmonic. Obviously this deformation was not unique because the effect of the symplectic transformation would remain the same upon adding a proper symplectic function to the deformation. Therefore we also considered adding a separate non-harmonic function to $\Omega$ (cf. (4.6)). We concluded that this modification must lead to new terms in $\Omega$, as shown in (4.7), but they contribute only to the Hesse potential by an additive contribution of the original non-harmonic function. However, when defining $\mathcal{H}^{(1)}$ we agreed that such additions should be included as a separate symplectic functions in the expansion of the Hesse potential in terms of independent functions. Hence this additive term will not affect $\mathcal{H}^{(1)}$. This aspect is essential for deriving the holomorphic anomaly equation. However, when the non-harmonic function is of first order in the deformation parameter, it will not contribute to the holomorphic anomaly equation, because it does not generate higher-order terms under the iteration, while (4.27) only receives contributions beyond the first order. The first-order contributions are instead governed by the separate equation (4.28). 
We will not analyze this issue in further detail here, but there is one type of deformation of the anomaly equation that is worth recalling. Suppose that on the right-hand side of (4.27) we change $h+4 \alpha \ln N$ by adding a function of the Kähler potential defined in (4.24) (which equals the Hesse potential with $\Omega=0$ ). This Kähler potential satisfies the following identities,

$$
\partial_{I} K=N_{I J} \overline{\mathcal{Y}}^{J}, \quad \partial_{I} \partial_{\bar{J}} K=N_{I J}, \quad D_{I} \partial_{J} K=0
$$

which all have a geometrical meaning. Adding a function of $K$ to $h+4 \alpha \ln N$ will introduce terms proportional to $N_{M P} \overline{\mathcal{Y}}^{P}$ or $N_{N P} \overline{\mathcal{Y}}^{P}$ on the right-hand side of (4.27) that cancel when contracted with the overall factor $\bar{F}_{I K L} N^{K M} N^{L N}$.

Finally, as is shown in (4.19), the transformation rule of $\omega$ has now acquired new terms of order $\alpha^{2}$. The derivatives of $\omega$ will thus receive corresponding contributions. In particular (4.13) and (4.15) will change. For the calculations in section 5 it is relevant to present the full expressions for the variations of $\omega_{I}$ and $\omega_{I J}$ up to order $\alpha^{3}$. In view of their length we have listed these equations in appendix C.

\section{$5 \quad$ Evaluating the third-order contributions}

There are good reasons for evaluating also the contributions of order $\alpha^{3}$. One of them is that $\Omega$ and $\mathcal{H}^{(1)}$ are no longer obviously 'partially harmonic' in higher orders. Another one is that the contributions of third order have never been fully worked out explicitly for the topological string.

Let us again start with (3.19), but now approximated to terms of order $\Omega^{3}$,

$$
\begin{aligned}
\tilde{\Omega}(\tilde{\mathcal{Y}}, \overline{\mathcal{Y}})= & \Omega-\mathrm{i}\left(\mathcal{Z}^{I J} \Omega_{I} \Omega_{J}-\overline{\mathcal{Z}}^{I J} \Omega_{\bar{I}} \Omega_{\bar{J}}\right) \\
& +\frac{2}{3}\left(F_{I J K} \mathcal{Z}^{I L} \Omega_{L} \mathcal{Z}^{J M} \Omega_{M} \mathcal{Z}^{K N} \Omega_{N}+\text { h.c. }\right) \\
& -2\left(\Omega_{I J} \mathcal{Z}^{I K} \Omega_{K} \mathcal{Z}^{J L} \Omega_{L}+\text { h.c. }\right)+4 \Omega_{I \bar{J}} \mathcal{Z}^{I K} \Omega_{K} \overline{\mathcal{Z}}^{J L} \Omega_{\bar{L}}+\mathcal{O}\left(\Omega^{4}\right),
\end{aligned}
$$

which must hold irrespective of the precise form of $\Omega$. To evaluate the right-hand side we must first determine $\Omega_{I}$ to order $\alpha^{2}$, which follows from (4.18),

$$
\begin{aligned}
\Omega_{I}= & \omega_{I}-\mathrm{i} \alpha F_{I J K} N^{J K} \\
& +2 \alpha\left[\omega_{I J K} N^{J K}+\mathrm{i}\left(\omega_{J K}+\bar{\omega}_{J K}\right) N^{J L} F_{I L M} N^{M K}\right] \\
& -\alpha^{2}\left[\mathrm{i} F_{I J K L M}-\frac{4}{3} F_{I J L N} N^{N P} F_{K M P}\right] N^{J K} N^{L M} \\
& +2 \alpha^{2}\left(F_{J K L M}-\bar{F}_{J K L M}\right) N^{J K} N^{L N} N^{M P} F_{I N P} \\
& +2 \mathrm{i} \alpha^{2}\left(F_{J K L} F_{M N P}+\bar{F}_{J K L} \bar{F}_{M N P}\right) N^{J M} N^{K N} N^{L Q} N^{P R} F_{I Q R}+\mathcal{O}\left(\alpha^{3}\right) \\
= & \omega_{I}-\mathrm{i} \alpha F_{I J K} N^{J K}+2 \alpha \omega_{I J K} N^{J K} \\
& +2 \mathrm{i} \alpha F_{I J K} N^{J M} N^{K N}\left[\omega_{M N}+\alpha F_{M P R} F_{N Q S} N^{P Q} N^{R S}-\mathrm{i} \alpha F_{M N P Q} N^{P Q}\right] \\
& -\alpha^{2}\left[\mathrm{i} F_{I J K L M}-\frac{4}{3} F_{I J L N} N^{N P} F_{K M P}\right] N^{J K} N^{L M}
\end{aligned}
$$




$$
\begin{aligned}
& +2 \mathrm{i} \alpha F_{I J K} N^{J M} N^{K N}\left[\bar{\omega}_{M N}+\alpha \bar{F}_{M P R} \bar{F}_{N Q S} N^{P Q} N^{R S}+\mathrm{i} \alpha \bar{F}_{M N P Q} N^{P Q}\right] \\
& +\mathcal{O}\left(\alpha^{3}\right) .
\end{aligned}
$$

We now observe that the above expression is no longer 'almost holomorphic' and thus deviates from the results obtained before. The troublesome terms are contained in the last line of (5.2), which turns out to be equal to

$$
2 \mathrm{i} \alpha F_{I J K} N^{J M} N^{K N} \Omega_{\bar{M} \bar{N}}+\mathcal{O}\left(\alpha^{3}\right),
$$

where we made use of the second equation in (4.14). It will be convenient to keep writing these non-holomorphic contributions in terms of non-holomorphic derivatives of $\Omega$. The crucial point to note is, however, that we have extracted an explicit factor of $\alpha$, whereas so far $\alpha$ appeared only implicitly in $\Omega$. As we will demonstrate shortly, one consequence of our analysis is that the Hesse potential will involve additional symplectic functions, but now multiplied by explicit powers of $\alpha$.

Substituting the above result (5.2) and the last two equations of (4.14) into (5.1), we obtain,

$$
\begin{aligned}
\tilde{\Omega}(\tilde{\mathcal{Y}}, \overline{\mathcal{Y}})- & \Omega(\mathcal{Y}, \overline{\mathcal{Y}}) \\
= & \left\{-\mathrm{i}\left(\omega_{I}-\mathrm{i} \alpha F_{I \bullet \bullet} N^{\bullet \bullet}\right) \mathcal{Z}^{I J}\left(\omega_{J}-\mathrm{i} \alpha F_{J \bullet \bullet} N^{\bullet \bullet}\right)\right. \\
& +\frac{2}{3} F_{I J K} \mathcal{Z}^{I L}\left(\omega_{L}-\mathrm{i} \alpha F_{L \bullet \bullet} N^{\bullet \bullet}\right) \mathcal{Z}^{J M}\left(\omega_{M}-\mathrm{i} \alpha F_{M \bullet \bullet} N^{\bullet \bullet}\right) \\
& \quad \times \mathcal{Z}^{K N}\left(\omega_{N}-\mathrm{i} \alpha F_{N \bullet \bullet} N^{\bullet \bullet}\right) \\
& -4 \mathrm{i} \alpha\left(\omega_{I}-\mathrm{i} \alpha F_{I \bullet \bullet} N^{\bullet \bullet}\right) \mathcal{Z}^{I J}\left[\omega_{J \bullet \bullet} N^{\bullet \bullet}+\mathrm{i} \omega_{K} \bullet N^{\bullet \bullet} F_{J \bullet \bullet} N^{\bullet K}\right] \\
& +2 \mathrm{i} \alpha^{2}\left(\omega_{I}-\mathrm{i} \alpha F_{I \bullet \bullet} N^{\bullet \bullet}\right) \mathcal{Z}^{I J}\left[\mathrm{i} F_{J K L M N}-\frac{4}{3} F_{J K M P} N^{P Q} F_{L N Q}\right] N^{K L} N^{M N} \\
& -4 \mathrm{i} \alpha^{2}\left(\omega_{I}-\mathrm{i} \alpha F_{I \bullet \bullet} N^{\bullet \bullet}\right) \mathcal{Z}^{I J} F_{J P Q} N^{M P} N^{N Q} N^{K L} F_{K L M N} \\
& +4 \alpha^{2}\left(\omega_{I}-\mathrm{i} \alpha F_{I \bullet \bullet} N^{\bullet \bullet}\right) \mathcal{Z}^{I J} F_{J R S} N^{M R} N^{Q S} N^{K N} N^{L P} F_{K L M} F_{N P Q} \\
& -2\left(\omega_{I}-\mathrm{i} \alpha F_{I \bullet \bullet} N^{\bullet \bullet}\right) \mathcal{Z}^{I J}\left(\omega_{J K}+\alpha F_{J M N} F_{K P Q} N^{M P} N^{N Q}-\mathrm{i} \alpha F_{J K \bullet \bullet} N^{\bullet \bullet}\right) \\
& \left.\quad \times \mathcal{Z}^{K L}\left(\omega_{L}-\mathrm{i} \alpha F_{L \bullet \bullet} N^{\bullet \bullet}\right)+\text { h.c. }\right\} \\
& +4 \alpha\left\{\Omega_{I} \mathcal{Z}^{I J} F_{J K L} N^{K M} N^{L N} \Omega_{\bar{M} \bar{N}}+\text { h.c. }\right\} \\
& -4 \alpha \Omega_{I} \mathcal{Z}^{I J} F_{J K L} N^{K M} N^{L N} \bar{F}_{P M N} \overline{\mathcal{Z}}^{P Q} \Omega_{\bar{Q}}+\mathcal{O}\left(\alpha^{4}\right) .
\end{aligned}
$$

The last two lines are not 'almost harmonic'. The first of these two lines arises as a result of the non-holomorphic terms noted in (5.3), and the last line originates from the manifestly non-harmonic term present at the end of the expression (5.1) (which has been included above upon replacing $\Omega_{I \bar{J}}$ by the corresponding expression given in (4.14)).

It is now straightforward to verify with the help of (3.20) that these two lines are precisely generated upon assuming that $\Omega$ will contain a term $-4 \alpha \Omega_{I J} N^{I K} N^{J L} \Omega_{\bar{K} \bar{L}}$ at this order of iteration. This is quite a non-trivial result, because we are not just rewriting the expression (5.1) that was originally expressed in terms of $\Omega$ and its derivatives, into a 
similar expression! Rather, as already mentioned, we have now extracted an explicit power of $\alpha$, whereas so far the parameter $\alpha$ only appeared implicitly in $\Omega$. This signals a new pattern that will become more manifest shortly.

Using the previous results of the transformation rules of the function $\omega$ and its derivatives, exhibited in (4.15) and (C.1), as well as the transformation rules (2.8) for multiple derivatives of the holomorphic function $F$, one can, after a fair amount of non-trivial manipulations, determine the expression for $\Omega(\mathcal{Y}, \overline{\mathcal{Y}})$, up to a symplectic function of $\mathcal{Y}$ and $\overline{\mathcal{Y}}$,

$$
\begin{aligned}
\Omega(\mathcal{Y}, \overline{\mathcal{Y}})=\alpha \ln & N \\
+\left\{\begin{array}{l}
\omega \\
+
\end{array}\right. & 2 \alpha N^{I J} \omega_{I J}-2 \alpha N^{I J} N^{K L}\left[\omega_{I K} \omega_{J L}-\alpha \omega_{I J K L}\right] \\
& +\frac{8}{3} \mathrm{i} \alpha^{2} F_{I J K} N^{I L} N^{J M} N^{K N} \omega_{L M N} \\
& -\alpha^{2}\left[\mathrm{i} F_{I J K L}-\frac{2}{3} F_{I K M} F_{J L N} N^{M N}\right] N^{I J} N^{K L} \\
& +4 \mathrm{i} \alpha^{2} N^{P Q}\left(F_{P Q I J}+\mathrm{i} F_{P I M} N^{M N} F_{Q J N}\right) N^{I K} N^{J L} \omega_{K L} \\
& -\frac{2}{3} \alpha^{3} N^{I J}\left[\mathrm{i} F_{I J K L M N} N^{K L} N^{M N}-4 F_{I J K L M} N^{K N} N^{L P} N^{M Q} F_{N P O}\right] \\
& +2 \alpha^{3}\left[N^{I J} F_{I J K L} N^{K P} N^{L Q} F_{P Q R S} N^{R S}\right. \\
& \left.+\frac{1}{3} F_{I J K L} N^{I M} N^{J N} N^{K P} N^{L Q} F_{M N P Q}\right] \\
& +4 \mathrm{i} \alpha^{3} F_{I J K L}\left[N^{I J} N^{K M} N^{L N} F_{M P Q} N^{P R} N^{Q S} F_{R S N}\right. \\
& \left.+N^{I M} N^{J N} N^{K P} N^{L Q} F_{M N R} N^{R S} F_{P Q S}\right] \\
& -2 \alpha^{3} F_{I J K} F_{L P Q} F_{R S T} F_{U V W} N^{I L} N^{J P} N^{K R} N^{Q U} N^{S V} N^{T W} \\
& \left.-\frac{4}{3} \alpha^{3} F_{I J K} F_{L P Q} F_{R S T} F_{U V W} N^{I L} N^{J R} N^{K U} N^{P S} N^{Q V} N^{T W}+\text { h.c. }\right\} \\
-4 & \alpha \Omega_{I J} N^{I K} N^{J L} \Omega_{\bar{K} \bar{L}}+\mathcal{O}\left(\alpha^{4}\right) .
\end{aligned}
$$

It is clear that the terms that are independent of the holomorphic function $\omega(\mathcal{Y})$ are becoming more and more numerous in higher orders. Note that the above result is 'almost harmonic', with the exception of the last term. Furthermore the 'almost harmonic' terms take again the form of a sum over 1PI diagrams.

In the limit $\alpha \rightarrow 0$ the expression (5.5) for $\Omega$ reduces to the original harmonic expression that we started from initially in (4.1). With the exception of the last term in (5.5), which will recombine with other terms in due course, the almost harmonic terms have to be included into the expression for the function that encodes the effective action. Hence they imply that the original non-harmonic modification $\ln N$ in (4.6) is incomplete and must be modified order-by-order by additional non-harmonic term. These terms will thus contribute to the effective action, where they are expected to encode non-local interactions associated with the massless modes.

We remind the reader that $\Omega$ is not a symplectic function and the next step is to determine the symplectic function $\mathcal{H}^{(1)}$ in third order of $\alpha$, which follows upon substitution of 
the above result for $\Omega$ into (3.18). Let us first concentrate on the terms that are not 'almost harmonic'. They originate from three different sources. First there is the last term in (5.5) (which appears with an additional factor 4 in $\mathcal{H}^{(1)}$ ), then there are explicit non-harmonic terms in the expression (3.18) for $\mathcal{H}^{(1)}$, and finally there are the non-holomorphic contributions in (5.2) that were summarized in (5.3), which induce corresponding modifications in $\mathcal{H}^{(1)}$. These three contibutions are

$$
\begin{aligned}
& -16 \alpha \Omega_{I J} N^{I K} N^{J L} \Omega_{\bar{K} \bar{L}} \\
& -16 \alpha \Omega_{I} N^{I J} F_{J M N} N^{M P} N^{N Q} \bar{F}_{P Q K} N^{K L} \Omega_{\bar{L}} \\
& -16 \mathrm{i} \alpha \Omega_{I} N^{I J} F_{J K L} N^{K M} N^{L N} \Omega_{\bar{M} \bar{N}}+\text { h.c. }
\end{aligned}
$$

and they combine into

$$
-16 \alpha\left(\Omega_{I J}+\mathrm{i} F_{I J M} N^{M P} \Omega_{P}\right) N^{I K} N^{J L}\left(\Omega_{\bar{K} \bar{L}}-\mathrm{i} \bar{F}_{K L N} N^{N Q} \Omega_{\bar{Q}}\right),
$$

which equals precisely $-16 \alpha$ times the non-harmonic symplectic function $\mathcal{G}_{1}(\mathcal{Y}, \overline{\mathcal{Y}})$ that has been listed in appendix $\mathrm{B}$, up to terms of order $\Omega^{3}$. The function $\mathcal{H}^{(1)}$ thus acquires the form

$$
\mathcal{H}^{(1)}(\mathcal{Y}, \overline{\mathcal{Y}} ; N)=4 \alpha \ln N+h(\mathcal{Y}, \overline{\mathcal{Y}})+\bar{h}(\overline{\mathcal{Y}}, \mathcal{Y})-16 \alpha \mathcal{G}_{1}(\mathcal{Y}, \overline{\mathcal{Y}})+\mathcal{O}\left(\alpha^{4}\right)
$$

where

$$
\begin{aligned}
& h(\mathcal{Y}, \overline{\mathcal{Y}})=4 \omega-4\left(\omega_{I}-\mathrm{i} \alpha F_{I K L} N^{K L}\right) N^{I J}\left(\omega_{J}-\mathrm{i} \alpha F_{J M N} N^{M N}\right) \\
& +8 \alpha \omega_{I J} N^{I J}-8 \alpha N^{I J} N^{K L}\left[\omega_{I K} \omega_{J L}-\alpha \omega_{I J K L}\right] \\
& +\frac{32}{3} \mathrm{i} \alpha^{2} F_{I J K} N^{I L} N^{J M} N^{K N} \omega_{L M N} \\
& +16 \mathrm{i} \alpha^{2} N^{\bullet \bullet}\left(F_{\bullet \bullet I J}+\mathrm{i} F_{\bullet I M} N^{M N} F_{\bullet J N}\right) N^{I K} N^{J L} \omega_{K L} \\
& -16 \alpha \omega_{I \bullet \bullet} N^{\bullet \bullet} N^{I J}\left(\omega_{J}-\mathrm{i} \alpha F_{J} \bullet N^{\bullet \bullet}\right) \\
& +8\left(\omega_{I}-\mathrm{i} \alpha F_{I \bullet \bullet} N^{\bullet \bullet}\right) N^{I J} \omega_{J K} N^{K L}\left(\omega_{L}-\mathrm{i} \alpha F_{L \bullet \bullet} N^{\bullet \bullet}\right) \\
& +\frac{8}{3} \mathrm{i} F_{I J K} N^{I L} N^{J M} N^{K N}\left(\omega_{L}-\mathrm{i} \alpha F_{L \bullet \bullet} N^{\bullet \bullet}\right) \\
& \times\left(\omega_{M}-\mathrm{i} \alpha F_{M \bullet \bullet} N^{\bullet \bullet}\right)\left(\omega_{N}-\mathrm{i} \alpha F_{N \bullet \bullet} N^{\bullet \bullet}\right) \\
& -16 \mathrm{i} \alpha\left(\omega_{I}-\mathrm{i} \alpha F_{I \bullet \bullet} N^{\bullet \bullet}\right) N^{I J} F_{J K L} N^{K M} N^{L N} \omega_{M N} \\
& +8 \mathrm{i} \alpha^{2}\left(\omega_{I}-\mathrm{i} \alpha F_{I \bullet \bullet} N^{\bullet \bullet}\right) N^{I J} \\
& \times\left(F_{J K L \bullet \bullet}+\frac{4}{3} \mathrm{i} F_{J K M \bullet} N^{M N} F_{L N \bullet}\right) N^{K L} N^{\bullet \bullet} \\
& -8 \mathrm{i} \alpha\left(\omega_{I}-\mathrm{i} \alpha F_{I \bullet \bullet} N^{\bullet \bullet}\right) N^{I J}\left(F_{J K \bullet \bullet}+\mathrm{i} F_{J P \bullet} N^{P Q} F_{K Q} \bullet\right) N^{\bullet \bullet} \\
& \times N^{K L}\left(\omega_{L}-\mathrm{i} \alpha F_{L \bullet \bullet} N^{\bullet \bullet}\right) \\
& -16 \alpha^{2}\left(\omega_{I}-\mathrm{i} \alpha F_{I \bullet \bullet} N^{\bullet \bullet}\right) N^{I J} F_{J K L} N^{K M} N^{L N} \\
& \times\left(F_{M N \bullet \bullet}+\mathrm{i} F_{M P \bullet} N^{P Q} F_{N Q \bullet}\right) N^{\bullet \bullet} \\
& -4 \alpha^{2}\left[\mathrm{i} F_{I J K L}-\frac{2}{3} F_{I K M} F_{J L N} N^{M N}\right] N^{I J} N^{K L}
\end{aligned}
$$




$$
\begin{aligned}
& -\frac{8}{3} \alpha^{3} N^{I J}\left[\mathrm{i} F_{I J K L M N} N^{K L} N^{M N}-4 F_{I J K L M} N^{K N} N^{L P} N^{M Q} F_{N P Q}\right] \\
& +8 \alpha^{3}\left[N^{I J} F_{I J K L} N^{K P} N^{L Q} F_{P Q R S} N^{R S}\right. \\
& \left.\quad+\frac{1}{3} F_{I J K L} N^{I M} N^{J N} N^{K P} N^{L Q} F_{M N P Q}\right] \\
& +16 \mathrm{i} \alpha^{3} F_{I J K L}\left[N^{I J} N^{K M} N^{L N} F_{M P Q} N^{P R} N^{Q S} F_{R S N}\right. \\
& \left.\quad+N^{I M} N^{J N} N^{K P} N^{L Q} F_{M N R} N^{R S} F_{P Q S}\right] \\
& -8 \alpha^{3} F_{I J K} F_{L P Q} F_{R S T} F_{U V W} N^{I L} N^{J P} N^{K R} N^{Q U} N^{S V} N^{T W} \\
& -\frac{16}{3} \alpha^{3} F_{I J K} F_{L P Q} F_{R S T} F_{U V W} N^{I L} N^{J R} N^{K U} N^{P S} N^{Q V} N^{T W} \\
& +\mathcal{O}\left(\alpha^{4}\right) .
\end{aligned}
$$

As before the $\omega(\mathcal{Y})$ will transform holomorphically such that its explicit transformation rule follows from (5.9) upon making the substitution $N_{I J} \rightarrow \mathrm{i} \mathcal{Z}^{I J}$. We have verified by explicit calculation that this is indeed the case, which provides an explicit check on the calculation.

At this point one can again determine the holomorphic anomaly equation following the same steps as before. As it turns out the result coincides with (4.27), but now valid up to order $\alpha^{4}$. This can be seen as an indication that the holomorphic anomaly equation will not acquire further corrections in higher orders.

\section{$6 \quad$ Summary and conclusions}

Based on the observation that the duality transformations act differently on the function that encodes the effective action than on the topological free energy, we have proposed a conceptual framework based on the Hesse potential of real special geometry to understand the relation between the two. Subsequently we have studied the Hesse potential by iteration for a generic effective action, first starting from a Wilsonian effective action and subsequently by considering the effect of non-harmonic deformations. The Hesse potential decomposes into an infinite series of symplectic functions and we established that the topological string free energy could reside in precisely one of them. This function is then subject to the holomorphic anomaly equation, irrespective of its dynamical content.

The results of an explicit iteration of the genus $g \leq 3$ topological string free energy fully confirms the correctness of the proposal. We should again stress that we concentrate on the generic features of this relationship, rather than on specific models. The relations that we find are thus universal, but it is not assumed that the resulting expression for the topological string free energy will have an actual realization as a topological string model. This is the reason that we do not make contact with specific aspects of the topological string, such as the wave function approach and the issue of background dependence [35].

One implication of our result is that we are also able to relate the non-holomorphic terms associated to the effective action to the ones that appear in the topological string free energy. This is perhaps not so surprising in view of the fact that there is a qualitative 
relation between the pinching of a cycle that decreases the genus of the Riemann surface in the topological string and the integration over massless modes in the effective action! But it is important to realize that, while our construction demonstrates how to construct the topological string free energy from a given effective action, the inverse is clearly not possible because the effective action is equivalent to the full Hesse potential, while the topological string free energy constitutes only part of the Hesse potential.

At several occasions we already mentioned that the results of this paper are consistent with our previous work $[15,16]$, where we analyzed the same issues by using a variety of different strategies. It is therefore of interest to compare the present results with the results of the past. To highlight some interesting issues we therefore reconsider the earlier results on the FHSV model, which were based on imposing the exact S- and T-dualities of this model on the effective action. There we used a slightly different perturbative procedure and we worked in a parametrization based on special coordinates. Subsequently we determined the Hesse potential by iteration, in a way that is similar to what was done in the present paper. We then discovered that the Hesse potential did indeed contain terms that cannot belong to the topological string free energy at genus-2, because they do not depend (anti-)holomorphically on the topological string coupling. As we now know, those are the contributions that do not belong to the function $\mathcal{H}^{(1)}$, but at that stage such a systematic classification was not available. Nevertheless, the terms that did depend (anti-)holomorphically on the topological string coupling were consistent with the results obtained from the FHSV topological string [27], except that the proportionality constant remained ambiguous in view of the fact that the corresponding expression was duality invariant, so that its leading contribution could be changed by a corresponding change in the effective action where we had only imposed the requirement of invariance. Interestingly, the present approach which emphasizes covariance rather than invariance, clarifies this result. To appreciate all this we have summarized some of details of the derivation of the genus- 2 FHSV topological string free energy in appendix E.

Finally we wish to return to the issue of BPS black hole entropy in supersymmetric theories with eight supercharges, which formed a major motivation for the present work. In $[31,33]$ a general formula for BPS black hole entropy was given based on Wald's definition of black hole entropy [36], which was covariant under dualities and incorporated the higher-derivative corrections to the Weyl multiplet that we already referred to in section 1. (Incidentally, there is now increasing evidence that other higher-derivative couplings will not contribute to BPS black hole entropy by virtue of certain non-renormalization theorems [3, 4].) The formula of [33] was reinterpreted in [29] in terms of a mixed partition function which was subsequently related to the topological string. However, this relationship depended crucially on the assumption that the topological free energy and the function that encodes the supergravity action are directly related, or perhaps even identical! As we have been trying to emphasize in this paper, the topological string does capture certain string amplitudes that should also follow from the effective action. But this does not imply that the topological string and the action are given by the same function.

We should perhaps add here that it is possible to present the supergravity input in the form of the Hesse potential (analogous to converting a Lagrangian into a Hamiltonian 
description), for which one can define a modified black hole partition function associated with the canonical ensemble [22]. This would offer an effective way to make contact with the topological string, were it not for the fact that the black hole solutions from which one starts in supergravity are, by definition, solutions of the full effective action. Therefore they should involve the full Hesse potential, which, as we have shown in this paper, consists of an infinite series of symplectic functions of which just one will correspond to the topological string free energy. Finally we note that the work of this paper pertains specifically to theories with eight supercharges, while a substantial part of the literature on BPS black holes is based on theories with sixteen supercharges (although often treated in a reduction to eight supercharges). An extension of the work of this paper for theories to sixteen supersymmetries should therefore be of interest.

\section{Acknowledgments}

We acknowledge helpful discussions with Murad Alim, Michele Cirafici, Edi Gava, Thomas Grimm, Babak Haghighat, Albrecht Klemm, Thomas Mohaupt, Kumar Narain, Hirosi Ooguri, Álvaro Osorio, Ashoke Sen, Samson Shatashvili, Marcel Vonk, Edward Witten and Maxim Zabzine. The work of G.L.C. is partially funded by Fundação para a Ciência e a Tecnologia (FCT/Portugal) through project PEst-OE/EEI/LA0009/2013 and through the grants PTDC/MAT/119689/2010 and EXCL/MAT-GEO/0222/2012. The work of B.d.W. is supported by the ERC Advanced Grant no. 246974, "Supersymmetry: a window to non-perturbative physics". This work is also supported by the COST action MP1210 "The String Theory Universe".

We thank our respective institutes for hospitality during the course of this work. S.M. thanks the Alexander von Humboldt Stiftung for a reinvitation grant which enabled her to visit the Max Planck Institut, München, and Dieter Lüst for offering hospitality. We also thank the Max Planck Institut für Gravitationsphysik (Albert-Einstein-Institute) for hospitality extended to us during the completion of this work.

\section{A Non-holomorphic deformation of special geometry}

In this appendix we prove the following theorem.

\section{A.1 Theorem}

Given a Lagrangian $\mathcal{L}(\phi, \dot{\phi})$ depending on $n$ coordinates $\phi^{i}$ and $n$ velocities $\dot{\phi}^{i}$, with corresponding Hamiltonian $\mathcal{H}(\phi, \pi)=\dot{\phi}^{i} \pi_{i}-\mathcal{L}(\phi, \dot{\phi})$, there exists a description in terms of complex coordinates $x^{i}=\frac{1}{2}\left(\phi^{i}+\mathrm{i} \dot{\phi}^{i}\right)$ and a complex function $F(x, \bar{x})$, such that,

$$
\begin{aligned}
2 \operatorname{Re} x^{i} & =\phi^{i}, \\
2 \operatorname{Re} F_{i}(x, \bar{x}) & =\pi_{i}, \quad \text { where } F_{i}=\frac{\partial F(x, \bar{x})}{\partial x^{i}} .
\end{aligned}
$$

The function $F(x, \bar{x})$ is defined up to an anti-holomorphic function and can be decomposed into a holomorphic and a purely imaginary non-harmonic function,

$$
F(x, \bar{x})=F^{(0)}(x)+2 \mathrm{i} \Omega(x, \bar{x}) .
$$


The equivalence transformations take the form,

$$
F^{(0)} \rightarrow F^{(0)}+g(x), \quad \Omega \rightarrow \Omega-\operatorname{Im} g(x),
$$

which results in $F(x, \bar{x}) \rightarrow F(x, \bar{x})+\bar{g}(\bar{x})$.

The Lagrangian can then be expressed in terms of $F$ and $\Omega$,

$$
\mathcal{L}=4[\operatorname{Im} F-\Omega]
$$

so that the Hamiltonian takes the form

$$
\mathcal{H}=-4[\operatorname{Im} F-\Omega]+2 \pi_{i} \operatorname{Im} x^{i} .
$$

This expression is identical to the expression for the Hesse potential given in (2.4), up to an overall minus sign. Alternatively the Hamiltonian can be written as

$$
\mathcal{H}=-\mathrm{i}\left(x^{i} \bar{F}_{\bar{\imath}}-\bar{x}^{\bar{\imath}} F_{i}\right)-4 \operatorname{Im}\left[F^{(0)}-\frac{1}{2} x^{i} F_{i}^{(0)}\right]-2\left(2 \Omega-x^{i} \Omega_{i}-\bar{x}^{\bar{\imath}} \Omega_{\bar{\imath}}\right),
$$

where $F_{i}=\partial F / \partial x^{i}, \bar{F}_{\bar{\imath}}=\partial \bar{F} / \partial \bar{x}_{\bar{\imath}}$, and similarly for the functions $F^{(0)}$ and $\Omega$. When the function $F^{(0)}(x)$ is homogeneous of second degree, the second term will vanish. The third term is a measure of the deviation from homogeneity of $\Omega$. This decomposition is known from the entropy function for BPS black holes [22].

Furthermore, the $2 n$-vector $\left(x^{i}, F_{i}\right)$ turns out to define a complexification of the phasespace coordinates $\left(\phi^{i}, \pi_{i}\right)$ that transforms precisely as $\left(\phi^{i}, \pi_{i}\right)$ under canonical (symplectic) reparametrizations,

$$
\left(\begin{array}{c}
x^{i} \\
F_{i}(x, \bar{x})
\end{array}\right) \longrightarrow\left(\begin{array}{c}
\tilde{x}^{i} \\
\tilde{F}_{i}(\tilde{x}, \overline{\tilde{x}})
\end{array}\right)=\left(\begin{array}{cc}
U^{i}{ }_{j} & Z^{i j} \\
W_{i j} & V_{i}^{j}
\end{array}\right)\left(\begin{array}{c}
x^{j} \\
F_{j}(x, \bar{x})
\end{array}\right),
$$

where the real matrix is an element of $\operatorname{Sp}(2 n, \mathbb{R})$. Observe that for the real part of the vector $\left(x^{i}, F_{i}\right)$, the above transformation is the standard canonical transformation on coordinates and momenta. The equation (A.7) is integrable so that the symplectic transformation leads to new functions $\tilde{F}^{(0)}$ and $\tilde{\Omega}$.

\section{A.2 Proof}

The proof of this theorem proceeds as follows. First note the following complex vectors,

$$
x^{i}=\frac{1}{2}\left(\phi^{i}+\mathrm{i} \frac{\partial \mathcal{H}}{\partial \pi_{i}}\right), \quad y_{i}=\frac{1}{2}\left(\pi_{i}-\mathrm{i} \frac{\partial \mathcal{H}}{\partial \phi^{i}}\right)
$$

constructed out of two canonical pairs, one comprising the variables $\phi^{i}, \pi_{i}$ and the other one the derivatives of the Hamiltonian, which transform in the same way under canonical transformations (here we use that the Hamiltonian transforms as a function under canonical transformations). 
In view of the inverse Legendre relation, $\dot{\phi}^{i}=\partial \mathcal{H} / \partial \pi_{i}$, the complex $x^{i}$ in (A.8) coincide with the $x^{i}$ defined previously. Furthermore, when writing the Lagrangian as a function of the $x^{i}$ and $\bar{x}^{\bar{\imath}}$, it follows that

$$
\frac{\partial \mathcal{L}(x, \bar{x})}{\partial x^{i}}=-2 \mathrm{i} y_{i}
$$

Here we used that the Legendre transformation leading to the Hamiltonian yields $\partial \mathcal{L} / \partial \phi^{i}=$ $-\partial \mathcal{H} / \partial \phi^{i}$ (where on the right-hand side $\pi_{i}$ is kept constant and on the left-hand side $\dot{\phi}^{i}$ is kept constant). Observe that we did not make use of the equations of motion.

Subsequently we write $\mathcal{L}$ as the sum of a harmonic and a non-harmonic function,

$$
\mathcal{L}=-2 \mathrm{i}\left[F^{(0)}(x)-\bar{F}^{(0)}(\bar{x})\right]+4 \Omega(x, \bar{x})=4[\operatorname{Im} F-\Omega],
$$

so that (A.9) reads

$$
y_{i}=\frac{\partial}{\partial x^{i}}\left[F^{(0)}(x)+2 \mathrm{i} \Omega(x, \bar{x})\right] .
$$

Thus $y_{i}=\partial_{i} F(x, \bar{x})$ with $F(x, \bar{x})=F^{(0)}(x)+2 \mathrm{i} \Omega(x, \bar{x})$, up to an arbitrary anti-holomorphic function, and $\operatorname{Re} y_{i}=\pi_{i}$. The Hamiltonian then follows from (A.5), which leads to the expression (A.6). Hence we now have shown that $\left(x^{i}, F_{i}\right)$ equals the vector $\left(x^{i}, y_{i}\right)$ which transforms under canonical transformations according to (A.7).

What remains to be proven is that the result of the transformation (A.7) is integrable. The vector $\left(x^{i}, y_{i}\right)$ transforms according to (A.7) into a vector $\left(\tilde{x}^{i}, \tilde{y}_{i}\right)$ while the Hamiltonian, which depends on $\left(x^{i}+\bar{x}^{i}, y_{i}+\bar{y}_{i}\right)$, transforms as a function under canonical transformations, so that $\tilde{\mathcal{H}}\left(\tilde{x}^{i}+\overline{\tilde{x}}^{i}, \tilde{y}_{i}+\overline{\tilde{y}}_{i}\right)=\mathcal{H}\left(x^{i}+\bar{x}^{i}, y_{i}+\bar{y}_{i}\right)$. The dual quantities $\left(\tilde{x}^{i}, \tilde{y}_{i}\right)$ and the new Hamiltonian $\tilde{\mathcal{H}}$ will satisfy the same relation as the original quantities. The new Lagrangian $\tilde{\mathcal{L}}$ which follows from an inverse Legendre transformation of the new Hamiltonian, will depend on $\tilde{x}^{i}$ and $\overline{\tilde{x}}^{i}$. Applying the same steps as before we then find the new function $\tilde{F}(\tilde{x}, \overline{\tilde{x}})$.

There is one subtlety here and that is that the decomposition of the function $F$ into $F^{(0)}$ and $\Omega$ is ambiguous. The ambiguity is resolved by noting that the symplectic transformation (A.7) can also be applied to the the vector $\left(x^{i}, F_{i}^{(0)}(x)\right)$. In that case the new function $F^{(0)}$ can be determined separately, as the holomorphic case is known to be integrable, and it is given in (2.7), up to a constant and terms linear in $\tilde{x}^{i}$. The latter terms can be determined explicitly, for instance, by using that $F^{(0)}-\frac{1}{2} x^{i} F_{i}^{(0)}$ transforms as a function under duality. Having determined the functions $\tilde{F}^{(0)}$ and $\tilde{F}$, the non-harmonic function $\tilde{\Omega}$ follows. This completes the proof of the theorem.

\section{A.3 Corollary}

Let us derive the well-known result (see, for instance, [37]) that the first-order derivative of the Lagrangian with respect to some parameter (such as a coupling constant) transforms as a function under symplectic transformations (A.7). We denote this parameter by $g$ and note that $\partial_{g} \mathcal{H}(\phi, \pi ; g)$ transforms as a function under canonical transformations (which do not depend on $g$, but they act on $g$-dependent quantities as shown in (A.7)) for any value 
of $g$. Subsequently, take the derivative of the Hamiltonian with respect to $g$ keeping $\phi^{i}$ and $\pi_{i}$ fixed. Consequently one derives

$$
\frac{\partial \mathcal{H}(\phi, \pi ; g)}{\partial g}=\left(\pi_{i}-\frac{\partial \mathcal{L}(\phi, \dot{\phi} ; g)}{\partial \dot{\phi}^{i}}\right) \frac{\partial \dot{\phi}^{i}}{\partial g}-\frac{\partial \mathcal{L}(\phi, \dot{\phi} ; g)}{\partial g}=-\frac{\partial \mathcal{L}(\phi, \dot{\phi} ; g)}{\partial g}
$$

which proves the assertion.

\section{B The symplectic functions $\mathcal{H}_{i}^{(a)}$ for $a \geq 2$ and some other functions that do not initially appear in $\mathcal{H}$}

Here we collect the explicit results for various functions $\mathcal{H}_{i}^{(a)}$ that appear in (3.16). These functions have been determined by iteration in orders of $\Omega$ and its derivatives. We present the terms of the iterative expansion up to $\mathcal{O}\left(\Omega^{5}\right)$. At the end of this appendix we will be presenting two more functions, $\mathcal{G}_{1}$ and $\mathcal{G}_{2}$, that did not initially show up in the iterative procedure for the Hesse potential carried out in this paper. These will only be given up to order $\Omega^{3}$. Note that here and elsewhere we only use indices $\bar{I}, \bar{J}, \ldots$ when they are necessary. For instance, we will write $F_{I J K}$ and $\bar{F}_{I J K}$ because $F$ is holomorphic and $\bar{F}$ is anti-holomorphic, so that there is no need for using holomorphic or anti-holomorphic indices, whereas for the derivatives of the real quantity $\Omega$ we write $\Omega_{I}$ and $\Omega_{\bar{I}}$ to distinguish holomorphic and anti-holomorphic derivatives. The reason is that $N^{I J}$ has no unique assignment of (anti)holomorphic indices, so that there will never be a consistent pattern of contractions based on holomorphic and anti-holomorphic indices. Denoting $(N \Omega)^{I} \equiv$ $N^{I J} \Omega_{J}$ and $(N \bar{\Omega})^{I} \equiv N^{I J} \Omega_{\bar{J}}$, we have obtained the following expressions,

$$
\begin{aligned}
\mathcal{H}^{(2)}= & 8 N^{I J} \Omega_{I} \Omega_{\bar{J}}-16\left[\Omega_{I J}(N \bar{\Omega})^{I}(N \Omega)^{J}+\Omega_{I \bar{J}}(N \bar{\Omega})^{I}(N \bar{\Omega})^{J}+\text { h.c. }\right] \\
& -8 \mathrm{i}\left[F_{I J K}(N \bar{\Omega})^{I}(N \Omega)^{J}(N \Omega)^{K}-\text { h.c. }\right] \\
& +\frac{16}{3} \mathrm{i}\left[\left(F_{I J K L}+3 \mathrm{i} F_{I J M} N^{M N} F_{N K L}\right)(N \Omega)^{I}(N \Omega)^{J}(N \Omega)^{K}(N \bar{\Omega})^{L}-\text { h.c. }\right] \\
& +16\left[\Omega_{I J K}(N \Omega)^{I}(N \Omega)^{J}(N \bar{\Omega})^{K}+\text { h.c. }\right] \\
& +16\left[\left(\Omega_{I J \bar{K}}+\mathrm{i} F_{I J M} N^{M N} \Omega_{N \bar{K}}\right)\left((N \Omega)^{I}(N \Omega)^{J}(N \Omega)^{K}+2(N \Omega)^{I}(N \bar{\Omega})^{J}(N \bar{\Omega})^{K}\right)\right] \\
& +16\left[\left(\Omega_{\bar{I} \bar{J} K}-\mathrm{i} \bar{F}_{I J M} N^{M N} \Omega_{\bar{N} K}\right)\left((N \bar{\Omega})^{I}(N \bar{\Omega})^{J}(N \bar{\Omega})^{K}+2(N \bar{\Omega})^{I}(N \Omega)^{J}(N \Omega)^{K}\right)\right] \\
& +32\left[\Omega_{I K} N^{K L} \Omega_{L J}(N \Omega)^{I}(N \bar{\Omega})^{J}+\text { h.c. }\right] \\
& +32 \Omega_{I K} N^{K L} \Omega_{\bar{L} \bar{J}}(N \Omega)^{I}(N \bar{\Omega})^{J} \\
& +16 \mathrm{i}\left[F_{I J K} N^{K L} \Omega_{L M}\left((N \Omega)^{I}(N \Omega)^{J}(N \bar{\Omega})^{M}+2(N \Omega)^{M}(N \Omega)^{I}(N \bar{\Omega})^{J}\right)-\text { h.c. }\right] \\
& +16 \mathrm{i}\left[F_{I J K} N^{K L} \Omega_{\bar{L} \bar{M}}(N \Omega)^{I}(N \Omega)^{J}(N \bar{\Omega})^{M}-\text { h.c. }\right] \\
& +8(N \Omega)^{I}(N \Omega)^{J} F_{I J M} N^{M N} \bar{F}_{N K L}(N \bar{\Omega})^{K}(N \bar{\Omega})^{L} \\
& +32\left[(N \Omega)^{I} \Omega_{I J} N^{J K} \Omega_{K \bar{L}}(N \Omega)^{L}+\text { h.c. }\right] \\
& +32\left[(N \bar{\Omega})^{I} \Omega_{I J} N^{J K} \Omega_{K \bar{L}}(N \bar{\Omega})^{L}+\text { h.c. }\right] \\
& +32\left[(N \Omega)^{I} \Omega_{I J} N^{J K} \Omega_{\bar{K} L}(N \Omega)^{L}+\text { h.c. }\right]
\end{aligned}
$$




$$
\begin{aligned}
& +16 \mathrm{i}\left[(N \Omega)^{I}(N \Omega)^{J} F_{I J K} N^{K L} \Omega_{\bar{L} M}(N \Omega)^{M}-\text { h.c. }\right] \\
& +32\left[(N \Omega)^{I} \Omega_{I \bar{J}} N^{J K} \Omega_{\bar{K} L}(N \bar{\Omega})^{L}+\text { h.c. }\right] \\
& +32\left[(N \Omega)^{I} \Omega_{I \bar{J}} N^{J K} \Omega_{K \bar{L}}(N \bar{\Omega})^{L}\right], \\
& \mathcal{H}_{1}^{(3)}=-\frac{8}{3} \mathrm{i} F_{I J K}(N \bar{\Omega})^{I}(N \bar{\Omega})^{J}(N \bar{\Omega})^{K} \\
& +8 \mathrm{i} F_{I J K}(N \bar{\Omega})^{I}(N \bar{\Omega})^{J} N^{K L} \\
& \times\left[2 \Omega_{\bar{L} \bar{M}}(N \bar{\Omega})^{M}+2 \Omega_{\bar{L} M}(N \Omega)^{M}-\mathrm{i} \bar{F}_{L M N}(N \bar{\Omega})^{M}(N \bar{\Omega})^{N}\right], \\
& \mathcal{H}_{2}^{(3)}=8\left(\Omega_{I J}+\mathrm{i} F_{I J K}(N \Omega)^{K}\right)(N \bar{\Omega})^{I}(N \bar{\Omega})^{J} \\
& -\frac{8}{3} \mathrm{i}\left(F_{I J K L}+3 \mathrm{i} F_{M(I J} N^{M N} F_{K L) N}\right) \\
& \times\left[3(N \Omega)^{I}(N \Omega)^{J}(N \bar{\Omega})^{K}(N \bar{\Omega})^{L}-2(N \bar{\Omega})^{I}(N \bar{\Omega})^{J}(N \bar{\Omega})^{K}(N \Omega)^{L}\right] \\
& -\frac{16}{3} \Omega_{I J K}\left(3(N \bar{\Omega})^{I}(N \bar{\Omega})^{J}(N \Omega)^{K}-(N \bar{\Omega})^{I}(N \bar{\Omega})^{J}(N \bar{\Omega})^{K}\right) \\
& -16 \Omega_{I J \bar{K}}(N \bar{\Omega})^{I}(N \bar{\Omega})^{J}(N \bar{\Omega})^{K} \\
& -16 \mathrm{i} F_{I J K} N^{K L} \Omega_{L M} \\
& \times\left[-(N \bar{\Omega})^{I}(N \bar{\Omega})^{J}(N \bar{\Omega})^{M}+(N \bar{\Omega})^{I}(N \bar{\Omega})^{J}(N \Omega)^{M}+2(N \bar{\Omega})^{I}(N \Omega)^{J}(N \bar{\Omega})^{M}\right] \\
& -16(N \bar{\Omega})^{I} \Omega_{I J} N^{J K} \Omega_{K L}(N \bar{\Omega})^{L} \\
& -32(N \Omega)^{I}\left(\Omega_{I J}+\mathrm{i} F_{I J K}(N \Omega)^{K}\right) N^{J L}\left(\Omega_{\bar{L} \bar{M}}-\mathrm{i} \bar{F}_{L M N}(N \bar{\Omega})^{N}\right)(N \Omega)^{M} \\
& +16 \mathrm{i}(N \Omega)^{I}(N \Omega)^{J} F_{I J K} N^{K L}\left(\Omega_{\bar{L} \bar{M}}-\mathrm{i} \bar{F}_{L M N}(N \bar{\Omega})^{\bar{N}}\right)(N \Omega)^{M} \\
& -16(N \Omega)^{I} \Omega_{\bar{I} J} N^{J K} \Omega_{K \bar{M}}(N \Omega)^{M} \\
& -32(N \bar{\Omega})^{I}\left(\Omega_{I J}+\mathrm{i} F_{I J K}(N \Omega)^{K}\right) N^{J L} \Omega_{\bar{L} M}(N \Omega)^{M} \\
& -16 \mathrm{i}(N \bar{\Omega})^{I}(N \bar{\Omega})^{J} F_{I J K} N^{K L} \Omega_{L \bar{M}}(N \bar{\Omega})^{M} \text {, } \\
& \mathcal{H}_{3}^{(3)}=-16 \Omega_{I \bar{J}}(N \bar{\Omega})^{I}(N \Omega)^{J} \\
& +16\left[2(N \bar{\Omega})^{I}(N \Omega)^{J}\left(\Omega_{I K} N^{K L} \Omega_{L \bar{J}}+\Omega_{I \bar{J} M}(N \Omega)^{M}\right)\right. \\
& +(N \bar{\Omega})^{I} \Omega_{I \bar{J}} N^{J K}\left(\mathrm{i} F_{K L M}(N \Omega)^{L}(N \Omega)^{M}+2 \Omega_{K L}(N \Omega)^{L}+2 \Omega_{K \bar{L}}(N \bar{\Omega})^{L}\right) \\
& \left.+2 \mathrm{i}(N \bar{\Omega})^{I}(N \Omega)^{J} F_{I J K} N^{K L} \Omega_{L \bar{M}}(N \Omega)^{M}+\text { h.c. }\right], \\
& \mathcal{H}_{1}^{(4)}=32(N \bar{\Omega})^{I}\left(\Omega_{I J}+\mathrm{i} F_{I J K}(N \Omega)^{K}\right) N^{J L}\left(\Omega_{\bar{L} \bar{M}}-\mathrm{i} \bar{F}_{\bar{L} \bar{M} \bar{N}}(N \bar{\Omega})^{N}\right)(N \Omega)^{M}, \\
& \mathcal{H}_{2}^{(4)}=32(N \Omega)^{I} \Omega_{\bar{I} J} N^{J K} \Omega_{\bar{K} L}(N \bar{\Omega})^{L} \\
& \mathcal{H}_{3}^{(4)}=8 F_{I J M} N^{M N} \bar{F}_{N K L}(N \bar{\Omega})^{I}(N \bar{\Omega})^{J}(N \Omega)^{K}(N \Omega)^{L}, \\
& \mathcal{H}_{4}^{(4)}=-\frac{4}{3} \mathrm{i}\left(F_{I J K L}+3 \mathrm{i} F_{M I J} N^{M N} F_{K L N}\right)(N \bar{\Omega})^{I}(N \bar{\Omega})^{J}(N \bar{\Omega})^{K}(N \bar{\Omega})^{L}, \\
& \mathcal{H}_{5}^{(4)}=-16 \mathrm{i} F_{I J K} N^{K Q} \Omega_{\bar{Q} L}(N \bar{\Omega})^{L}(N \bar{\Omega})^{I}(N \bar{\Omega})^{J}, \\
& \mathcal{H}_{6}^{(4)}=-16 \mathrm{i} F_{I J K} N^{K L}\left(\Omega_{\bar{L} \bar{M}}-\mathrm{i} \bar{F}_{L M N}(N \bar{\Omega})^{N}\right)(N \bar{\Omega})^{I}(N \bar{\Omega})^{J}(N \Omega)^{M} \text {, } \\
& \mathcal{H}_{7}^{(4)}=16\left(\Omega_{I J \bar{K}}+\mathrm{i} F_{I J L} N^{L M} \Omega_{M \bar{K}}\right)(N \bar{\Omega})^{I}(N \bar{\Omega})^{J}(N \Omega)^{K} \text {, }
\end{aligned}
$$




$$
\begin{aligned}
& \mathcal{H}_{8}^{(4)}=32(N \bar{\Omega})^{I}\left(\Omega_{I J}+\mathrm{i} F_{I J K}(N \Omega)^{K}\right) N^{J L} \Omega_{\bar{L} M}(N \bar{\Omega})^{M}, \\
& \mathcal{H}_{9}^{(4)}=-16 \mathrm{i}(N \bar{\Omega})^{I}(N \bar{\Omega})^{J} F_{I J K} N^{K L} \Omega_{\bar{L} M}(N \bar{\Omega})^{M} .
\end{aligned}
$$

As indicated above there are also other functions that do not initially appear in $\mathcal{H}$. We give two examples below up to terms of order $\Omega^{3}$.

$$
\begin{aligned}
& \mathcal{G}_{1}=\left(\Omega_{I J}+\mathrm{i} F_{I J M} N^{M P} \Omega_{P}\right) N^{I K} N^{J L}\left(\Omega_{\bar{K} \bar{L}}-\mathrm{i} \bar{F}_{K L N} N^{N Q} \Omega_{\overline{\mathrm{Q}}}\right), \\
& \mathcal{G}_{2}=\Omega_{I \bar{J}} N^{I L} N^{J K} \Omega_{K \bar{L}} .
\end{aligned}
$$

Note that the functions $\mathcal{G}_{1,2}$ take the form of 1PI connected diagrams, whereas the functions $\mathcal{H}_{i}^{(0)}$ do not.

\section{Transformation rules of $\omega_{I}$ and $\omega_{I J}$ to order $\alpha^{2}$}

In this appendix we list the transformation rules of some of the derivatives of the function $\omega$. For the first four multiple derivatives those were already given in (4.13) and (4.15) to order $\alpha$. However, the transformation rule of $\omega$ itself is known to order $\alpha^{2}$ (cf. (4.19)) so that also the derivatives can be determined in that order. In section 5 we in fact need the transformation rules for $\omega_{I}$ and $\omega_{I J}$ to order $\alpha^{3}$. In view of their length we display these transformations in this appendix. The results read as follows,

$$
\begin{aligned}
& \tilde{\omega}_{I}=\left[\mathcal{S}^{-1}\right]_{I}^{J}\left[\omega_{J}+\alpha F_{J K L} \mathcal{Z}^{K L}+2 \mathrm{i} \alpha \omega_{J K L} \mathcal{Z}^{K L}\right. \\
& -2 \mathrm{i}\left(\omega_{J \bullet}+\alpha F_{J \bullet K L} \mathcal{Z}^{K L}\right) \mathcal{Z}^{\bullet \bullet}\left(\omega_{\bullet}+\alpha F_{\bullet M N} \mathcal{Z}^{M N}\right) \\
& +\mathrm{i}\left(\omega_{\bullet}+\alpha F_{\bullet K L} \mathcal{Z}^{K L}\right) \mathcal{Z}^{\bullet \bullet} F_{J \bullet \bullet} \mathcal{Z}^{\bullet \bullet}\left(\omega_{\bullet}+\alpha F_{\bullet M N} \mathcal{Z}^{M N}\right) \\
& -2 \mathrm{i} \alpha \mathcal{Z}^{K \bullet} F_{J \bullet \bullet} \mathcal{Z}^{\bullet L}\left[\omega_{K L}-F_{K L \bullet} \mathcal{Z}^{\bullet \bullet}\left(\omega_{\bullet}+\alpha F_{\bullet M N} \mathcal{Z}^{M N}\right)\right] \\
& -2 \mathrm{i} \alpha^{2} \mathcal{Z}^{K \bullet} F_{J \bullet \bullet} \mathcal{Z}^{\bullet L}\left(F_{K L M N}-F_{K M \bullet} \mathcal{Z}^{\bullet \bullet} F_{L N \bullet}\right) \mathcal{Z}^{M N} \\
& \left.+\mathrm{i} \alpha^{2}\left[F_{J K L M N}-\frac{4}{3} F_{J K M \bullet} \mathcal{Z}^{\bullet \bullet} F_{L N \bullet}\right] \mathcal{Z}^{K L} \mathcal{Z}^{M N}\right]+\mathcal{O}\left(\alpha^{3}\right), \\
& \tilde{\omega}_{I J}=\left[\mathcal{S}^{-1}\right]^{K}{ }_{(I}\left[\mathcal{S}^{-1}\right]^{L}{ }_{J} \\
& \times\left[\omega_{K L}-F_{K L M} \mathcal{Z}^{M N}\left(\omega_{N}+\alpha F_{N P Q} \mathcal{Z}^{P Q}\right)\right. \\
& +\alpha\left(F_{K L M N}-F_{K M P} F_{L N Q} \mathcal{Z}^{P Q}\right) \mathcal{Z}^{M N} \\
& +2 \mathrm{i} \alpha\left[\left(\omega_{K L P Q}-F_{K L \bullet} \mathcal{Z}^{\bullet \bullet} \omega_{\bullet P Q}\right) \mathcal{Z}^{P Q}-2\left(\omega_{K} \bullet \mathcal{Z}^{\bullet \bullet} F_{L \bullet \bullet} \mathcal{Z}^{\bullet \bullet}\right)\right] \\
& -2 \mathrm{i}\left(\omega_{K}+\alpha F_{K \bullet M N} \mathcal{Z}^{M N}\right) \mathcal{Z}^{\bullet \bullet}\left(\omega_{L}+\alpha F_{L \bullet P Q} \mathcal{Z}^{P Q}\right) \\
& +2 \mathrm{i} F_{K L \bullet} \mathcal{Z}^{\bullet \bullet}\left(\omega_{\bullet \bullet}+\alpha F_{\bullet \bullet M N} \mathcal{Z}^{M N}\right) \mathcal{Z}^{\bullet \bullet}\left(\omega_{\bullet}+\alpha F_{\bullet P Q} \mathcal{Z}^{P Q}\right) \\
& -2 \mathrm{i}\left(\omega_{K L \bullet}+\alpha F_{K L \bullet M N} \mathcal{Z}^{M N}\right) \mathcal{Z}^{\bullet \bullet}\left(\omega_{\bullet}+\alpha F_{\bullet P Q} \mathcal{Z}^{P Q}\right) \\
& +4 \mathrm{i}\left(\omega_{K \bullet}+\alpha F_{K \bullet M N} \mathcal{Z}^{M N}\right) \mathcal{Z}^{\bullet \bullet} F_{L \bullet \bullet} \mathcal{Z}^{\bullet \bullet}\left(\omega_{\bullet}+\alpha F_{\bullet} P Q \mathcal{Z}^{P Q}\right) \\
& +\mathrm{i}\left(\omega_{\bullet}+\alpha F_{\bullet M N} \mathcal{Z}^{M N}\right) \mathcal{Z}^{\bullet \bullet}\left[F_{\bullet K L \bullet}-2 F_{\bullet K \bullet} \mathcal{Z}^{\bullet \bullet} F_{\bullet} L_{\bullet}\right] \mathcal{Z}^{\bullet \bullet}\left(\omega_{\bullet}+\alpha F_{\bullet} P Q \mathcal{Z}^{P Q}\right) \\
& -\mathrm{i} F_{R S T}\left(\mathcal{Z}^{R \bullet} F_{\bullet K L}\right)\left[\mathcal{Z}^{S \bullet}\left(\omega_{\bullet}+\alpha F_{\bullet M N} \mathcal{Z}^{M N}\right)\right]\left[\mathcal{Z}^{T \bullet}\left(\omega_{\bullet}+\alpha F_{\bullet} P Q \mathcal{Z}^{P Q}\right)\right] \\
& +4 \mathrm{i} \alpha\left(F_{X \bullet \bullet} \mathcal{Z}^{\bullet \bullet} F_{K \bullet \bullet} \mathcal{Z}^{\bullet \bullet}\right) \mathcal{Z}^{X \bullet}\left(\omega_{L \bullet}+\alpha F_{L \bullet M N} \mathcal{Z}^{M N}\right)
\end{aligned}
$$




$$
\begin{aligned}
& +4 \mathrm{i} \alpha\left(F_{X K \bullet \bullet} \mathcal{Z}^{\bullet \bullet} F_{L \bullet \bullet} \mathcal{Z}^{\bullet \bullet}\right) \mathcal{Z}^{X \bullet}\left(\omega_{\bullet}+\alpha F_{\bullet M N} \mathcal{Z}^{M N}\right) \\
& -4 \mathrm{i} \alpha\left(F_{X \bullet \bullet} \mathcal{Z}^{\bullet \bullet} F_{K \bullet \bullet} \mathcal{Z}^{\bullet \bullet}\right) \mathcal{Z}^{X \bullet} F_{\bullet L \bullet} \mathcal{Z}^{\bullet \bullet}\left(\omega_{\bullet}+\alpha F_{\bullet M N} \mathcal{Z}^{M N}\right) \\
& -2 \mathrm{i} \alpha\left(F_{K L R S}-F_{K L \bullet} \mathcal{Z}^{\bullet \bullet} F_{\bullet R S}-2 F_{K \bullet R} \mathcal{Z}^{\bullet \bullet} F_{L \bullet S}\right) \mathcal{Z}^{R T} \mathcal{Z}^{S U} \\
& \times\left[\omega_{T U}-F_{T U} \mathcal{Z}^{\bullet \bullet}\left(\omega_{\bullet}+\alpha F_{\bullet X Y} \mathcal{Z}^{X Y}\right)\right] \\
& +\mathrm{i} \alpha^{2}\left[F_{K L M N \bullet \bullet} \mathcal{Z}^{\bullet \bullet}-4\left(F_{K M N \bullet \bullet} \mathcal{Z}^{\bullet \bullet} F_{L \bullet \bullet} \mathcal{Z}^{\bullet \bullet}\right)\right] \mathcal{Z}^{M N} \\
& +2 \mathrm{i} \alpha^{2}\left(\mathcal{Z}^{M \bullet} F_{K \bullet \bullet} \mathcal{Z}^{\bullet N}\right)\left[F_{M N P Q}-F_{M P \bullet} \mathcal{Z}^{\bullet \bullet} F_{\bullet N Q}\right]\left(\mathcal{Z}^{P \bullet} F_{L \bullet \bullet} \mathcal{Z}^{\bullet Q}\right) \\
& -2 \mathrm{i} \alpha^{2}\left(\mathcal{Z}^{\bullet \bullet} F_{K \bullet \bullet} \mathcal{Z}^{\bullet \bullet} F_{X \bullet \bullet}\right) \mathcal{Z}^{X Y}\left(F_{Y \bullet \bullet} \mathcal{Z}^{\bullet \bullet} F_{L \bullet \bullet} \mathcal{Z}^{\bullet \bullet}\right) \\
& +8 \mathrm{i} \alpha^{2} F_{K M N P} F_{Q R S} \mathcal{Z}^{M Q} \mathcal{Z}^{N R}\left(\mathcal{Z}^{P \bullet} F_{L \bullet \bullet} \mathcal{Z}^{\bullet S}\right) \\
& -2 \mathrm{i} \alpha^{2}\left(\mathcal{Z}^{M \bullet} F_{K L \bullet \bullet} \mathcal{Z}^{\bullet N}-2 \mathcal{Z}^{M \bullet} F_{K \bullet \bullet} \mathcal{Z}^{\bullet \bullet} F_{L \bullet \bullet} \mathcal{Z}^{\bullet N}\right) \\
& \times\left[F_{M N P Q}-F_{M P \bullet} \mathcal{Z}^{\bullet \bullet} F_{\bullet N Q}\right] \mathcal{Z}^{P Q} \\
& -\frac{4}{3} \mathrm{i} \alpha^{2}\left(F_{K L M N P} F_{Q R S}+F_{K M N P} F_{L Q R S}\right) \mathcal{Z}^{M Q} \mathcal{Z}^{N R} \mathcal{Z}^{P S} \\
& +2 \mathrm{i} \alpha^{2} F_{K L \bullet} \mathcal{Z}^{\bullet X} \mathcal{Z}^{M \bullet} F_{X \bullet \bullet} \mathcal{Z}^{\bullet N}\left(F_{M N P Q}-F_{M P \bullet} \mathcal{Z}^{\bullet \bullet} F_{\bullet N Q}\right) \mathcal{Z}^{P Q} \\
& -\mathrm{i} \alpha^{2} F_{K L \bullet} \mathcal{Z}^{\bullet \bullet}\left[F_{\bullet M N P Q}-\frac{4}{3} F_{M P \bullet \bullet} \mathcal{Z}^{\bullet \bullet} F_{\bullet N Q}\right] \mathcal{Z}^{M N} \mathcal{Z}^{P Q} \\
& \left.-2 \mathrm{i} \alpha^{2}\left(\mathcal{Z}^{\bullet \bullet} F_{\bullet} \bullet \mathcal{Z}^{\bullet \bullet} F_{\bullet M \bullet} \mathcal{Z}^{\bullet \bullet} F_{\bullet L \bullet} \mathcal{Z}^{\bullet \bullet} F_{\bullet N \bullet}\right) \mathcal{Z}^{M N}\right]+\mathcal{O}\left(\alpha^{3}\right) .
\end{aligned}
$$

Again we have sometimes represented indices by bullets whenever they are contracted in an ope or closed stringlike fashion and there is no ambiguity.

\section{Topological free energies for genus $g \leq 3$ that satisfy the holomorphic anomaly equation}

In this appendix we list the topological free energies $F^{(g)}(\mathcal{Y}, \overline{\mathcal{Y}})$ that follow from expanding $\mathcal{H}^{(1)}$, given in (4.20), order-by-order in $\alpha$. To order $\alpha^{3}$ we obtain

$$
\begin{aligned}
\mathcal{H}^{(1)}(\mathcal{Y}, \overline{\mathcal{Y}} ; N)= & 4\left[F^{(1)}(\mathcal{Y}, \overline{\mathcal{Y}})+\left(F^{(2)}(\mathcal{Y}, \overline{\mathcal{Y}})+F^{(3)}(\mathcal{Y}, \overline{\mathcal{Y}})+\text { h.c. }\right)\right] \\
& -16 \alpha \mathcal{G}_{1}(\mathcal{Y}, \overline{\mathcal{Y}})+\mathcal{O}\left(\alpha^{4}\right),
\end{aligned}
$$

where the function $\mathcal{G}_{1}$ is given in (B.14),. The symplectic functions $F^{(g)}(\mathcal{Y}, \overline{\mathcal{Y}})$ that appear at order $\alpha^{g}$ are given by (for $g=1,2,3$ )

$$
\begin{aligned}
F^{(1)}(\mathcal{Y}, \overline{\mathcal{Y}})= & \omega^{(1)}+\bar{\omega}^{(1)}+\alpha \ln \operatorname{det} N_{I J} \\
F^{(2)}(\mathcal{Y}, \overline{\mathcal{Y}})= & \omega^{(2)}-N^{I J}\left(\omega_{I}^{(1)}-\mathrm{i} \alpha F_{I K L} N^{K L}\right)\left(\omega_{J}^{(1)}-\mathrm{i} \alpha F_{J P Q} N^{P Q}\right) \\
& +2 \alpha N^{I J} \omega_{I J}^{(1)}-\alpha^{2}\left[\mathrm{i} N^{I J} N^{K L} F_{I J K L}-\frac{2}{3} N^{I J} F_{I K L} N^{K P} N^{L Q} F_{J P Q}\right] \\
F^{(3)}(\mathcal{Y}, \overline{\mathcal{Y}})= & \omega^{(3)}-2 N^{I J} \omega_{I}^{(2)} \omega_{J}^{(1)}+2 \omega_{I J}^{(1)} N^{I K} \omega_{K}^{(1)} N^{J L} \omega_{L}^{(1)} \\
& +\frac{2}{3} \mathrm{i} F_{I J K} N^{I P} \omega_{P}^{(1)} N^{J Q} \omega_{Q}^{(1)} N^{K L} \omega_{L}^{(1)} \\
& +\alpha\left[2 \mathrm{i} N^{I J} \omega_{I}^{(2)} F_{J K L} N^{K L}-4 N^{I J} \omega_{I K L}^{(1)} N^{K L} \omega_{J}^{(1)}\right. \\
& \quad-4 \mathrm{i} N^{I J} N^{K L} F_{I L M} N^{M N} \omega_{K N}^{(1)} \omega_{J}^{(1)}-2 \mathrm{i} F_{I J K L} N^{K L} N^{I P} \omega_{P}^{(1)} N^{J Q} \omega_{Q}^{(1)}
\end{aligned}
$$




$$
\begin{aligned}
& +2 F_{I K P} N^{K L} F_{L Q J} N^{P Q} N^{I R} \omega_{R}^{(1)} N^{J S} \omega_{S}^{(1)} \\
& \text { - } 4 \mathrm{i} \omega_{I J}^{(1)} N^{I K} \omega_{K}^{(1)} N^{J L} F_{L P Q} N^{P Q} \\
& +2 F_{I J K} N^{I P} \omega_{P}^{(1)} N^{J Q} \omega_{Q}^{(1)} N^{K R} F_{R S T} N^{S T} \\
& \left.+2 N^{I J} \omega_{I J}^{(2)}-2 N^{I J} N^{K L} \omega_{I K}^{(1)} \omega_{J L}^{(1)}\right] \\
& +\alpha^{2}\left[2 \mathrm{i} N^{I J} F_{I K L M N} N^{K L} N^{M N} \omega_{J}^{(1)}-4 N^{I J} N^{K L} F_{I L M} N^{M N} F_{K N P Q} N^{P Q} \omega_{J}^{(1)}\right. \\
& -\frac{8}{3} N^{I J} F_{I M N P} N^{M K} N^{N L} N^{P Q} F_{K L Q} \omega_{J}^{(1)} \\
& \text { - } 4 \mathrm{i} N^{I J} N^{K L} F_{I L M} N^{M N} F_{K R T} N^{R P} N^{T Q} F_{N P Q} \omega_{J}^{(1)} \\
& +4 \mathrm{i} N^{I J} \omega_{I M N}^{(1)} N^{M N} F_{J K L} N^{K L}-4 N^{I J} N^{M N} F_{I N P} N^{P Q} \omega_{M Q}^{(1)} F_{J K L} N^{K L} \\
& -2 \omega_{I J}^{(1)} N^{I K} F_{K P Q} N^{P Q} N^{J L} F_{L R S} N^{R S} \\
& -4 F_{I J M N} N^{M N} N^{I K} \omega_{K}^{(1)} N^{J L} F_{L R S} N^{R S} \\
& \text { - 4i } F_{I M N} N^{M P} F_{J P Q} N^{Q N} N^{I K} \omega_{K}^{(1)} N^{J L} F_{L R S} N^{R S} \\
& \text { - } 2 \text { i } F_{I J K} N^{I P} \omega_{P}^{(1)} N^{J Q} F_{Q S T} N^{S T} N^{K R} F_{R U V} N^{U V} \\
& +2 N^{I J} N^{K L} \omega_{I J K L}^{(1)}+\frac{8}{3} \mathrm{i} F_{I J K} N^{I L} N^{J P} N^{K Q} \omega_{L P Q}^{(1)} \\
& \left.+4 \mathrm{i} F_{I J R S} N^{I J} N^{R K} N^{S L} \omega_{K L}^{(1)}-4 F_{I P Q} F_{J R S} N^{I K} N^{J L} N^{P R} N^{Q S} \omega_{K L}^{(1)}\right] \\
& +\alpha^{3}\left[2 N^{I J} F_{I M N P Q} N^{M N} N^{P Q} F_{J K L} N^{K L}\right. \\
& +4 \mathrm{i} N^{I J} N^{P M} F_{I M N} N^{N Q} F_{P Q R S} N^{R S} F_{J K L} N^{K L} \\
& +\frac{8}{3} \mathrm{i} N^{I J} F_{I M N P} N^{M K} N^{N L} N^{P Q} F_{K L Q} F_{J R S} N^{R S} \\
& -4 N^{I J} N^{P M} F_{I M N} N^{N Q} F_{P T U} N^{T R} N^{U S} F_{Q R S} F_{J K L} N^{K L} \\
& +2 \mathrm{i} F_{I J M N} N^{M N} N^{I K} F_{K P Q} N^{P Q} N^{J L} F_{L R S} N^{R S} \\
& -2 F_{I M N} N^{M T} F_{J T U} N^{U N} N^{I K} F_{K P Q} N^{P Q} N^{J L} F_{L R S} N^{R S} \\
& -\frac{2}{3} F_{I J K} N^{I P} F_{P M N} N^{M N} N^{J Q} F_{Q S T} N^{S T} N^{K R} F_{R U V} N^{U V} \\
& -\frac{2}{3} \mathrm{i} F_{I J K L P Q} N^{I J} N^{K L} N^{P Q}+\frac{8}{3} N^{I J} F_{I J K L P} N^{K R} N^{L S} N^{P T} F_{R S T} \\
& +2 N^{I J} F_{I J K L} N^{K P} N^{L Q} F_{P Q R S} N^{R S} \\
& +\frac{2}{3} F_{I K P S} N^{I J} N^{K L} N^{P Q} N^{S R} F_{J L Q R} \\
& +4 \mathrm{i} N^{I J} F_{I J K L} N^{K P} N^{L Q} F_{P S T} N^{S U} N^{T V} F_{Q U V} \\
& +4 \mathrm{i} F_{I J K L} N^{I P} N^{J Q} N^{K R} N^{L S} F_{P Q U} N^{U V} F_{V R S} \\
& -2 F_{I J K} F_{L P Q} F_{R S T} F_{U V W} N^{I L} N^{J P} N^{K R} N^{Q U} N^{S V} N^{T W} \\
& \left.-\frac{4}{3} F_{I J K} F_{L P Q} F_{R S T} F_{U V W} N^{I L} N^{J R} N^{K U} N^{P S} N^{Q V} N^{T W}\right] \text {. }
\end{aligned}
$$


Here we expanded $\omega(\mathcal{Y})$ as $\omega(\mathcal{Y})=\sum_{n=1}^{\infty} \omega^{(n)}(\mathcal{Y})$, where we count $\omega^{(n)}(\mathcal{Y})$ as being of order $\alpha^{n}$, following (4.16). The non-holomorphicity of $F^{(g)}(\mathcal{Y}, \overline{\mathcal{Y}})$ is entirely contained in the quantities $N^{I J}$. Observe that $F^{(1)}$ is real, while the higher $F^{(g)}(g \geq 2)$ are not.

The expressions for $F^{(g)}$ given above were obtained by explicit construction and they satisfy the holomorphic anomaly equations (4.27) of perturbative topological string theory $(g \geq 2)$,

$$
\partial_{\bar{I}} F^{(g)}=\mathrm{i} \bar{F}_{I J K} N^{J M} N^{K N}\left[-2 \alpha D_{M} \partial_{N} F^{(g-1)}+\sum_{r=1}^{g-1} \partial_{M} F^{(r)} \partial_{N} F^{(g-r)}\right],
$$

where $D_{M}$ denotes the covariant derivative introduced in (4.23). The expression for $F^{(2)}$ has been obtained before by other methods [11, 26, 27] based on a direct integration of (D.3). Partial results for $F^{(3)}$ have been given in [11].

\section{E An application: the FHSV model}

In this appendix we illustrate our results in the context of the FHSV model [28] and compare them to earlier results obtained in [16] by means of a related but slightly different approach. Here we restrict ourselves to second order. In the type-II description, the FHSV model corresponds to the compactification on the Enriques Calabi-Yau three-fold, which is described as an orbifold $\left(\mathrm{T}^{2} \times \mathrm{K} 3\right) / \mathbb{Z}_{2}$, where $\mathbb{Z}_{2}$ is a freely acting involution. The massless sector of the four-dimensional theory comprises 11 vector supermultiplets, 12 hypermultiplets and the $N=2$ graviton supermultiplet. The classical moduli space of the vector multiplet sector equals the special-Kähler space,

$$
\mathcal{M}_{\text {vector }}=\frac{\mathrm{SL}(2)}{\mathrm{SO}(2)} \times \frac{\mathrm{O}(10,2)}{\mathrm{O}(10) \times \mathrm{O}(2)},
$$

which is encoded in the classical holomorphic function

$$
F^{(0)}(\mathcal{Y})=-\frac{\mathcal{Y}^{1} \mathcal{Y}^{a} \eta_{a b} \mathcal{Y}^{b}}{\mathcal{Y}^{0}}
$$

where $a, b=2, \ldots, 11$, and the symmetric matrix $\eta_{a b}$ is an $\mathrm{SO}(9,1)$ invariant metric of indefinite signature. The two factors of the special-Kähler space are associated with $T^{2} / \mathbb{Z}_{2}$ and the K3 fiber, and 'special' coordinates for these two spaces are denoted by $S=-\mathrm{i} \mathcal{Y}^{1} / \mathcal{Y}^{0}$ and $T^{a}=-\mathrm{i} \mathcal{Y}^{a} / \mathcal{Y}^{0}$. This leads to the following expression for $N \equiv \operatorname{det}\left[2 \operatorname{Im}\left[F_{I J}^{(0)}\right]\right]$ and $\left.\mathcal{H}\right|_{\Omega=0}$,

$$
N=c(S+\bar{S})^{10}\left((T+\bar{T})^{2}\right)^{2},\left.\quad \mathcal{H}\right|_{\Omega=0}=-(S+\bar{S})(T+\bar{T})^{2}\left|\mathcal{Y}^{0}\right|^{2},
$$

where $c$ is an irrelevant constant. Here we use the notation that $T^{2} \equiv T^{a} \eta_{a b} T^{b}$ and likewise for $|T|^{2}$. Observe that $N$ is not covariant under symplectic reparametrizations, while $\left.\mathcal{H}\right|_{\Omega=0}$ is covariant. Note that in the present approach we are making use of a specifc parametrization defined by (E.2). Therefore the covariance under symplectic reparametrizations is not always clear, and instead we may have to rely on the S- and T-duality invariances that we will discuss below. This was also the strategy used in $[15,16]$. 
Subsequently, we expand both $\omega(\mathcal{Y})$ and $\Omega(\mathcal{Y}, \overline{\mathcal{Y}})$ into powers of $\alpha$ as $\omega=\omega^{(1)}+\omega^{(2)}+$ $\mathcal{O}\left(\alpha^{3}\right)$ and $\Omega=\Omega^{(1)}+\Omega^{(2)}+\mathcal{O}\left(\alpha^{3}\right)$, respectively. Following the discussion in [16], we start with the expression for $\Omega^{(1)}$, known from threshold corrections and from the topological string side $[38,39]$. In the conventions of [15], it is given by

$$
\begin{aligned}
\Omega^{(1)}(\mathcal{Y}, \overline{\mathcal{Y}})=-\frac{1}{4 \pi}[ & \frac{1}{2} \ln \left[\eta^{24}(2 S) \Phi(T)\right]+\frac{1}{2} \ln \left[\eta^{24}(2 \bar{S}) \Phi(\bar{T})\right] \\
& \left.+2 \ln \left[(S+\bar{S})^{3}(T+\bar{T})^{2}\right]\right]
\end{aligned}
$$

It is invariant under S-duality transformations belonging to the $\Gamma(2)$ subgroup of $\operatorname{SL}(2 ; \mathbb{Z})$, and also invariant under the T-duality group $\mathrm{O}(10,2 ; \mathbb{Z})$, since $\Phi(T)$ is a holomorphic automorphic form of weight 4 [40], transforming under the T-duality transformation $T^{a} \rightarrow$ $T^{a}\left[T^{2}\right]^{-1}$ as

$$
\Phi(T) \rightarrow\left[T^{2}\right]^{4} \Phi(T) .
$$

We can now recast (E.4) in the form of (4.6),

$$
\Omega^{(1)}(\mathcal{Y}, \overline{\mathcal{Y}})=\omega(\mathcal{Y})+\bar{\omega}(\overline{\mathcal{Y}})-\frac{1}{8 \pi} \ln N+\Psi(\mathcal{Y}, \overline{\mathcal{Y}})
$$

so that $\alpha=-1 /(8 \pi)$ and $\beta=1$, with

$$
\begin{aligned}
\omega^{(1)}(\mathcal{Y}) & =-\frac{3}{2 \pi} \ln \eta^{2}(2 S)-\frac{1}{8 \pi} \ln \Phi(T)+\frac{1}{4 \pi} \ln \mathcal{Y}^{0}, \\
\Psi(\mathcal{Y}, \overline{\mathcal{Y}}) & =-\frac{1}{4 \pi} \ln \left[(S+\bar{S})(T+\bar{T})^{2}\left|\mathcal{Y}^{0}\right|^{2}\right],
\end{aligned}
$$

where we note that $\Psi(\mathcal{Y}, \overline{\mathcal{Y}})$ transforms as a function because it is equal to the logarithm of $\left.\mathcal{H}\right|_{\Omega=0}$, the classical part of the Hesse potential.

Next, we insert these expressions into $\Omega^{(2)}$. Here we recall that in the presence of a function $\Psi(\mathcal{Y}, \overline{\mathcal{Y}})$, the expression for $\Omega^{(2)}$ is not simply obtained by the second line of (4.18), but to this we also have to add $\beta$-dependent terms, as shown in (4.7). Thus, we have

$$
\begin{aligned}
\Omega^{2)}(\mathcal{Y}, \overline{\mathcal{Y}})= & {\left[\omega^{(2)}+2 \alpha N^{I J} \omega_{I J}^{(1)}-\alpha^{2}\left[\mathrm{i} F_{I J K L}-\frac{2}{3} F_{I K M} F_{J L N} N^{M N}\right] N^{I J} N^{K L}+\text { h.c. }\right] } \\
& +N^{I J}\left[2 \partial_{I}\left(\omega^{(1)}+\alpha \ln N\right) \Psi_{J}+\Psi_{I} \Psi_{J}+\text { h.c. }\right] .
\end{aligned}
$$

Then, direct evaluation of this results in

$$
\begin{aligned}
& \Omega^{(2)}=\left\{\omega^{(2)}+\frac{1}{\left(\mathcal{Y}^{0}\right)^{2}}\left[\frac{1}{64 \pi^{2}} G_{2}(2 S) \frac{\partial \ln \Phi(T)}{\partial T^{a}} \frac{\partial \ln \Phi(T)}{\partial T_{a}}-\frac{1}{32 \pi^{2}} G_{2}(2 S) \frac{\partial^{2} \ln \Phi(T)}{\partial T^{a} \partial T_{a}}\right]\right. \\
& +\frac{1}{\left(\mathcal{Y}^{0}\right)^{2}}\left[4 \hat{G}_{2}(2 S, 2 \bar{S}) \frac{\partial \Omega^{(1)}}{\partial T^{a}} \frac{\partial \Omega^{(1)}}{\partial T_{a}}+\frac{1}{32 \pi^{2}} \hat{G}_{2}(2 S, 2 \bar{S})\left(\frac{\partial^{2} \ln \Phi(T)}{\partial T^{a} \partial T_{a}}+\frac{\partial \ln \Phi(T)}{\partial T^{a}} \frac{\partial \ln \Phi(T)}{\partial T_{a}}\right)\right] \\
& \left.-\frac{1}{\left(\mathcal{Y}^{0}\right)^{2}}\left[G_{2}(2 S) \frac{\partial \Omega^{(1)}}{\partial T^{a}} \frac{\partial \Omega^{(1)}}{\partial T_{a}}+\frac{1}{4} \frac{\partial \ln \Phi(T)}{\partial T_{a}} \frac{\partial \Omega^{(1)}}{\partial T^{a}} \frac{\partial \Omega^{(1)}}{\partial S}\right]+\text { h.c. }\right\}
\end{aligned}
$$

where

$$
\begin{aligned}
G_{2}(2 S) & =\frac{1}{2} \partial_{S} \ln \eta^{2}(2 S) \\
\hat{G}_{2}(2 S, 2 \bar{S}) & =G_{2}(2 S)+\frac{1}{2(S+\bar{S})} .
\end{aligned}
$$


The first line of (E.9) contains purely holomorphic terms, while the second line contains terms that are invariant under S- and T-duality. The last line contains the terms that are neither holomorphic nor invariant under S- and T-dualities. They were already obtained in [15] by requiring invariance of the model under S- and T-duality, and thus were determined up to invariant terms. Here, the duality invariant terms are unambiguously determined and given by the second line of (E.9), as we just established. The reason is that the scheme presented in this paper ensures the validity of the holomorphic anomaly equation. This implies that invariant terms cannot be arbitrarily included, as we discussed in section 4. Earlier results obtained in $[15,16]$ are fully consistent with the ones given above.

Next, we compute the symplectic function $F^{(2)}$, which is constructed from $\Omega^{(2)}$ as follows. Recalling (E.8), we write $\Omega^{(2)}$ as $\Omega^{(2)}=\Delta+\bar{\Delta}$. Then, from (D.2) we infer the relation $F^{(2)}=\Delta-N^{I J} \Omega_{I}^{(1)} \Omega_{J}^{(1)}$, where however (and differently from (D.2)) $\Omega^{(1)}$ now also contains $\Psi(\mathcal{Y}, \overline{\mathcal{Y}})$, cf. (4.6). As we have observed in the text below (4.10), the terms depending on $\Psi$ cancel in the higher order result for $\mathcal{H}^{(1)}$, and therefore $F^{(2)}$ will not depend on $\Psi$. We obtain

$$
\begin{aligned}
F^{(2)}= & \omega^{(2)}+\frac{1}{\left(\mathcal{Y}^{0}\right)^{2}}\left[\frac{1}{64 \pi^{2}} G_{2}(2 S) \frac{\partial \ln \Phi(T)}{\partial T^{a}} \frac{\partial \ln \Phi(T)}{\partial T_{a}}-\frac{1}{32 \pi^{2}} G_{2}(2 S) \frac{\partial^{2} \ln \Phi(T)}{\partial T^{a} \partial T_{a}}\right] \\
& +\frac{1}{32 \pi^{2}\left(\mathcal{Y}^{0}\right)^{2}} \hat{G}_{2}(2 S, 2 \bar{S})\left(\frac{\partial^{2} \ln \Phi(T)}{\partial T^{a} \partial T_{a}}+\frac{\partial \ln \Phi(T)}{\partial T^{a}} \frac{\partial \ln \Phi(T)}{\partial T_{a}}\right) \\
& -\frac{3}{64 \pi^{2}\left(\mathcal{Y}^{0}\right)^{2}} \hat{G}_{2}(2 S, 2 \bar{S}) \frac{\partial \log \left[\Phi(T)\left[(T+\bar{T})^{2}\right]^{4}\right]}{\partial T_{a}} \frac{\partial \log \left[\Phi(T)\left[(T+\bar{T})^{2}\right]^{4}\right]}{\partial T^{a}} .
\end{aligned}
$$

The first line contains purely holomorphic terms, while the second and third lines are given in terms of non-holomorphic combinations that are S- and T-duality invariant. The holomorphic contributions in the first line should, however, be invariant as well. We can verify this by making use of the transformation rule (4.19) for $\omega^{(2)}$. We have checked that the first line is indeed invariant under S-duality, and we expect the same for T-duality. At this stage we are not able to give an explicit representation of $\omega^{(2)}$ as a function of $\mathcal{Y}^{0}, S$ and $T^{a}$ that generates the desired transformations. The expression for $F^{(2)}$ given above is in agreement with the finding of [27].

Open Access. This article is distributed under the terms of the Creative Commons Attribution License (CC-BY 4.0), which permits any use, distribution and reproduction in any medium, provided the original author(s) and source are credited.

\section{References}

[1] B. de Wit and A. Van Proeyen, Potentials and Symmetries of General Gauged $N=2$ Supergravity: Yang-Mills Models, Nucl. Phys. B 245 (1984) 89 [INSPIRE].

[2] E. Bergshoeff, M. de Roo and B. de Wit, Extended Conformal Supergravity, Nucl. Phys. B 182 (1981) 173 [INSPIRE]. 
[3] B. de Wit, S. Katmadas and M. van Zalk, New supersymmetric higher-derivative couplings: Full $N=2$ superspace does not count!, JHEP 01 (2011) 007 [arXiv:1010.2150] [INSPIRE].

[4] D. Butter, B. de Wit, S.M. Kuzenko and I. Lodato, New higher-derivative invariants in $N=2$ supergravity and the Gauss-Bonnet term, JHEP 12 (2013) 062 [arXiv:1307.6546] [INSPIRE].

[5] S. Cecotti, S. Ferrara and L. Girardello, Geometry of Type II Superstrings and the Moduli of Superconformal Field Theories, Int. J. Mod. Phys. A 4 (1989) 2475 [inSPIRE].

[6] P. Candelas and X. de la Ossa, Moduli Space of Calabi-Yau Manifolds, Nucl. Phys. B 355 (1991) 455 [INSPIRE].

[7] A. Strominger, Special geometry, Commun. Math. Phys. 133 (1990) 163 [InSPIRE].

[8] B. Craps, F. Roose, W. Troost and A. Van Proeyen, What is special Kähler geometry?, Nucl. Phys. B 503 (1997) 565 [hep-th/9703082] [INSPIRE].

[9] N. Seiberg, Observations on the Moduli Space of Superconformal Field Theories, Nucl. Phys. B 303 (1988) 286 [INSPIRE].

[10] E. Witten, Topological $\sigma$-models, Commun. Math. Phys. 118 (1988) 411 [InSPIRE].

[11] M. Bershadsky, S. Cecotti, H. Ooguri and C. Vafa, Kodaira-Spencer theory of gravity and exact results for quantum string amplitudes, Commun. Math. Phys. 165 (1994) 311 [hep-th/9309140] [INSPIRE].

[12] R. Gopakumar and C. Vafa, $M$ theory and topological strings. 1., hep-th/9809187 [INSPIRE].

[13] R. Gopakumar and C. Vafa, M theory and topological strings. 2., hep-th/9812127 [INSPIRE].

[14] B. Eynard and M. Mariño, A Holomorphic and background independent partition function for matrix models and topological strings, J. Geom. Phys. 61 (2011) 1181 [arXiv:0810.4273] [INSPIRE].

[15] G.L. Cardoso, B. de Wit and S. Mahapatra, Subleading and non-holomorphic corrections to $N=2$ BPS black hole entropy, JHEP 02 (2009) 006 [arXiv:0808.2627] [INSPIRE].

[16] G.L. Cardoso, B. de Wit and S. Mahapatra, BPS black holes, the Hesse potential and the topological string, JHEP 06 (2010) 052 [arXiv:1003.1970] [INSPIRE].

[17] I. Antoniadis, E. Gava, K.S. Narain and T.R. Taylor, Topological amplitudes in string theory, Nucl. Phys. B 413 (1994) 162 [hep-th/9307158] [INSPIRE].

[18] L.J. Dixon, V. Kaplunovsky and J. Louis, Moduli dependence of string loop corrections to gauge coupling constants, Nucl. Phys. B 355 (1991) 649 [INSPIRE].

[19] M. Bershadsky, S. Cecotti, H. Ooguri and C. Vafa, Holomorphic anomalies in topological field theories, Nucl. Phys. B 405 (1993) 279 [hep-th/9302103] [INSPIRE].

[20] G.L. Cardoso, B. de Wit and T. Mohaupt, Macroscopic entropy formulae and nonholomorphic corrections for supersymmetric black holes, Nucl. Phys. B 567 (2000) 87 [hep-th/9906094] [INSPIRE].

[21] G.L. Cardoso, B. de Wit and S. Mahapatra, Non-holomorphic deformations of special geometry and their applications, Springer Proc. Phys. 144 (2013) 1 [arXiv:1206.0577] [INSPIRE].

[22] G.L. Cardoso, B. de Wit, J. Käppeli and T. Mohaupt, Black hole partition functions and duality, JHEP 03 (2006) 074 [hep-th/0601108] [INSPIRE]. 
[23] D.S. Freed, Special Kähler manifolds, Commun. Math. Phys. 203 (1999) 31 [hep-th/9712042] [INSPIRE].

[24] D.V. Alekseevsky, V. Cortes and C. Devchand, Special complex manifolds, J. Geom. Phys. 42 (2002) 85 [math/9910091] [INSPIRE].

[25] V. Cortés, A Holomorphic representation formula for parabolic hyperspheres, Banach Center Publ. 57 (2002) 11 [math/0107037] [INSPIRE].

[26] M. Aganagic, V. Bouchard and A. Klemm, Topological Strings and (Almost) Modular Forms, Commun. Math. Phys. 277 (2008) 771 [hep-th/0607100] [INSPIRE].

[27] T.W. Grimm, A. Klemm, M. Mariño and M. Weiss, Direct Integration of the Topological String, JHEP 08 (2007) 058 [hep-th/0702187] [INSPIRE].

[28] S. Ferrara, J.A. Harvey, A. Strominger and C. Vafa, Second quantized mirror symmetry, Phys. Lett. B 361 (1995) 59 [hep-th/9505162] [INSPIRE].

[29] H. Ooguri, A. Strominger and C. Vafa, Black hole attractors and the topological string, Phys. Rev. D 70 (2004) 106007 [hep-th/0405146] [INSPIRE].

[30] B. de Wit and M.T. Grisaru, Compensating fields and anomalies, in Quantum Field Theory and Quantum Statistics, vol. 2, I.A. Batalin, C.J. Isham, G.A. Vilkovisky eds., (1987) 411.

[31] G.L. Cardoso, B. de Wit, J. Käppeli and T. Mohaupt, Stationary BPS solutions in $N=2$ supergravity with $R^{2}$ interactions, JHEP 12 (2000) 019 [hep-th/0009234] [INSPIRE].

[32] G.L. Cardoso, B. de Wit, J. Käppeli and T. Mohaupt, Asymptotic degeneracy of dyonic $N=4$ string states and black hole entropy, JHEP 12 (2004) 075 [hep-th/0412287] [INSPIRE].

[33] G.L. Cardoso, B. de Wit and T. Mohaupt, Corrections to macroscopic supersymmetric black hole entropy, Phys. Lett. B 451 (1999) 309 [hep-th/9812082] [INSPIRE].

[34] B. de Wit, N=2 electric-magnetic duality in a chiral background, Nucl. Phys. Proc. Suppl. 49 (1996) 191 [hep-th/9602060] [INSPIRE].

[35] E. Witten, Quantum background independence in string theory, hep-th/9306122 [INSPIRE].

[36] R.M. Wald, Black hole entropy is the Noether charge, Phys. Rev. D 48 (1993) 3427 [gr-qc/9307038] [INSPIRE].

[37] M.K. Gaillard and B. Zumino, Duality Rotations for Interacting Fields, Nucl. Phys. B 193 (1981) 221 [INSPIRE].

[38] J.A. Harvey and G.W. Moore, Exact gravitational threshold correction in the FHSV model, Phys. Rev. D 57 (1998) 2329 [hep-th/9611176] [INSPIRE].

[39] A. Klemm and M. Mariño, Counting BPS states on the enriques Calabi-Yau, Commun. Math. Phys. 280 (2008) 27 [hep-th/0512227] [INSPIRE].

[40] R.E. Borcherds, Automorphic forms with singularities on Grassmannians, Invent. Math. 132 (1998) 491 [alg-geom/9609022]. 